AES/PE/11-31 Title: Gravity-Enhanced Transfer between Fracture and Matrix in Solvent-Based Enhanced Oil Recovery

Date: Aug-2011 Student name: Siavash Kahrobaei 
Title

Gravity-Enhanced Transfer between Fracture and Matrix in Solvent-Based Enhanced Oil Recovery

Author(s) $\quad$ : $\quad$ Siavash Kahrobaei

Date

Professor(s)

August 2011

TA Report number Prof. Dr. J. Bruining / Dr. Ir. R. Farajzadeh AES/PE/11-31

Postal Address

Section for Petroleum Engineering Department of Applied Earth Sciences Delft University of Technology P.O. Box 5028 The Netherlands

Telephone

(31) 152781328 (secretary)

Telefax

(31) 152781189

Copyright (C)2011 Section for Petroleum Engineering

All rights reserved.

No parts of this publication may be reproduced, Stored in a retrieval system, or transmitted, In any form or by any means, electronic, Mechanical, photocopying, recording, or otherwise, Without the prior written permission of the Section for Petroleum Engineering. 


\section{Gravity-enhanced transfer between fracture and matrix in solvent-based enhanced oil recovery}

Siavash Kahrobaei

August 2011 
This thesis is approved by:

Supervisors: Prof. Dr. J. Bruining Dr. Ir. R. Farajzadeh

and committee members:

Prof. Dr. J. Bruining CiTG Faculty, Technische Universiteit Delft

Dr. Ir. R. Farajzadeh CiTG Faculty, Technische Universiteit Delft

Dr. S. Rudolph CiTG Faculty, Technische Universiteit Delft

Dr. S. Suicmez Shell, EOR Department

Ir. A. Ameri CiTG Faculty, Technische Universiteit Delft 


\section{Contents}

1 Introduction $\quad 9$

1.1 Introduction . . . . . . . . . . . . . . . . 1

1.2 Literature Survey . . . . . . . . . . . . . . . . . . . . 3

1.2.1 Laboratory studies on mass transfer between matrix and fracture ................... 3

1.2.2 Numerical studies on mass transfer between matrix and fracture .................... . . 6

1.3 Objectives of the project $\ldots \ldots \ldots \ldots \ldots$

2 Formulation 9

2.1 Physical Model . . . . . . . . . . . . . . . . . . . . 99 9

2.2 Governing Equations . . . . . . . . . . . . . . . . . 11

2.2 .1 Continuity Equation . . . . . . . . . . . . . . 12

2.2 .2 Darcy's Law . . . . . . . . . . . . . . . . . . . . 13

2.2 .3 Transport Equation . . . . . . . . . . . . . . . . 13

2.3 Dimensionless form of the equations . . . . . . . . . . . 13

3 Soak Experiment: Principles and Procedure 16

3.1 Soak Experiment: Principles . . . . . . . . . . . . . 17

3.2 Fluids and Experimental Conditions _. . . . . . . . . . 18

3.3 Data Acquisition and Analysis . . . . . . . . . . . . . 19

4 Experimental Results 22

4.1 Averaging the four slices for each time step . . . . . . . . . 22

4.2 Pentane-Decalin . . . . . . . . . . . . . . . . 27

4.2.1 CT numbers at different horizons along the core . . . . 27

4.2.2 Comparison of CT numbers in horizontal and vertical sections in different times . . . . . . . . . . 31 
4.2.3 Averaging the CT-numbers along the core . . . . . . 37

4.2.4 Saturation Calculation . . . . . . . . . . . . . 40

4.2 .5 Recovery . . . . . . . . . . . . . . . . . 41

4.2 .6 Visualization of the process . . . . . . . . . . . . 44

4.3 Hexadecane-Decalin . . . . . . . . . . . . . . . . 48

4.3.1 CT numbers at different horizons along the core . . . . 48

4.3.2 Comparison of CT numbers in horizontal and vertical sections in different time steps . . . . . . . . . . . 50

4.3.3 Averaging the CT-numbers along the core . . . . . . . 55

4.3.4 Saturation Calculation . . . . . . . . . . . . . 56

4.3 .5 Recovery . . . . . . . . . . . . . . . 56 56

4.3 .6 Visualization of the process _ . . . . . . . . . . 59

4.4 Eicosane-Decalin . . . . . . . . . . . . . . . . . 62

4.4.1 Averaging the CT-numbers along the core . . . . . . 62

4.4 .2 Saturation Calculation . . . . . . . . . . . . . . . 63

4.4 .3 Recovery . . . . . . . . . . . . . . . . . . . . . 64

4.4.4 Visualization of the process . . . . . . . . . . 65

4.5 Hexadecane-Pentane . . . . . . . . . . . . . . . 68

4.5.1 Averaging the CT-numbers along the core . . . . . . 68

4.5.2 Saturation Calculation . . . . . . . . . . . . . . 69

4.5.3 Recovery . . . . . . . . . . . . . . . . . . 70

4.5.4 Visualization of the process . . . . . . . . . . 71

5 Simulation $\quad 74$

5.1 History matching of soak experiments . . . . . . . . . . 74

5.1.1 History match of experiment with Pentane-Decalin . . 77

5.1.2 History match of experiment with Hexadecane-Decalin 82

5.1.3 History match of experiment with Hexadecane-Pentane 84

5.2 Scaling Numbers . . . . . . . . . . . . . . . . . 86

6 Summary and Conclusions $\quad 88$

A Theoretical Background $\quad 96$

A.1 Gravity Drainage and diffusion . . . . . . . . . . . 96

A.2 Miscible Displacement . . . . . . . . . . . . . . . . 97

A.3 Diffusion . . . . . . . . . . . . . . . . . . . . 97

A.3.1 Fick's Law . . . . . . . . . . . . . . . . . . . . 98 
B Principals of Computerized Tomographic Imaging 99

B.1 Theory . . . . . . . . . . . . . . . . . . . . . 100

B.2 Line Integrals and Projection _ . . . . . . . . . . . 103

B.3 Image reconstruction . . . . . . . . . . . . . . . . . . . 104

B.3.1 Algebraic method . . . . . . . . . . . . . . . . . . 104

B.3.2 Radon transform . . . . . . . . . . . . . . . . 106

B.4 CT numbers . . . . . . . . . . . . . . . . . . . 111

C Saturation Procedure 112 


\section{List of Figures}

3.1 Schematic drawing of the glass jar containing the core in the experimental set-up . . . . . . . . . . . 17

4.1 Four different CT-images from the middle of the core (top) and their averaged image (bottom) . . . . . . . . . 23

4.2 Comparison of CT-numbers in three different positions along the core in a same time step . . . . . . . . . . . . . . 24

4.3 Average CT numbers of the core and the surrounding fracture 25

4.4 Average image of the core and the surrounding fracture . . . . 25

4.5 Comparison of the averaged CT numbers and the original CT numbers in different horizons along the core . . . . . . . . 26

4.6 Schematic drawing of position of different horizons along the

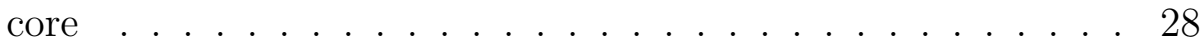

4.7 CT-numbers in three different horizontal position 23 minutes after the start of the experiment . . . . . . . . . . . . 29

4.8 Schematic drawing of position of different horizons along the

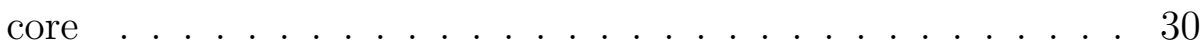

4.9 CT-numbers in three different vertical position 23 minutes after the start of the experiment . . . . . . . . . . . 30

4.10 CT-numbers in three different vertical position 1256 minutes after the start of the experiment . . . . . . . . . . . . 31

4.11 CT numbers at top of the core in different time steps. Green represents initial condition, which has been shown for each plot. Blue represents the current condition. . . . . . . . . . . . 32

4.12 CT numbers at middle of the core in different times. Green represents initial condition, which has been shown for each plot. Blue represents the current condition. . . . . . . . . . . . 33 
4.13 Variation of CT numbers at bottom of the core in different times. Green represents initial condition, which has been shown for each plot. Blue represents the current condition. . . 34

4.14 CT numbers at left side of the core in different time steps. Green represents initial condition, which has been shown for each plot. Blue represents the current condition. . . . . . . . . 35

4.15 Variation of CT numbers at middle section of the core in different times. Green represents initial condition, which has been shown for each plot. Blue represents the current condition. . . 36

4.16 Variation of CT numbers at right of the core in different times. Green represents initial condition, which has been shown for each plot. Blue represents the current condition. . . . . . . . 37

4.17 An example of cropped core image, taken in one of our experiments . . . . . . . . . . . . . . . . . 38

4.18 Variation of averaged CT numbers along the core in different time steps - Pentane-Decalin system . . . . . . . . . . . . 39

4.19 Variation of average of solvent saturation along the core in different time steps - Pentane-Decalin system . . . . . . . . . . 41

4.20 Recovery profile of Pentane . . . . . . . . . . . . . . . . . . 42

4.21 CT number Vs. different composition of Decalin and Pentane 43

4.22 Pentane Recovery and last point recovery obtained from the bulk ..................... . . 43

4.23 CT-images of the penetration of Decalin from the fracture into the core saturated with Pentane. Note that he blue color is Pentane inside the core and yellow color is Decalin. . . . . . . 47

4.24 CT-numbers in three different horizontal position 62 minutes after the start of the experiment . . . . . . . . . . . 48

4.25 CT-numbers in three different vertical position 62 minutes after the start of the experiment . . . . . . . . . . . . . . 49

$4.26 \mathrm{CT}$ numbers at top of the core in different times . . . . . . . . 50 4.27 CT numbers at middle of the core in different times . . . . . . 51 $4.28 \mathrm{CT}$ numbers at bottom of the core in different times . . . . . . 51 4.29 CT numbers at left side of the core in different times . . . . . 53 4.30 CT numbers at middle section of the core in different time steps 53 4.31 CT numbers at right side of the core in different times . . . . 54 4.32 Averaged CT numbers along the core in different times - HexadecaneDecalin system . . . . . . . . . . . . . . 55 
4.33 Average of solvent saturation along the core in different times - Hexadecane-Decalin system . . . . . . . . . . . . . 56

4.34 Recovery profile of hexadecane . . . . . . . . . . . . . . . 57

4.35 CT number Vs. different composition of Decalin and hexadecane 58

4.36 Hexadecane Recovery and last point recovery obtained from the bulk. By last point recovery we mean that at the end of the process, the jar is opened and the fluid inside the jar was stirred to obtain a homogenous mixture and then the composition of this mixture can be determined using a calibration curve and the CT-number of the mixture. . . . . . . . . . . . 59

4.37 Visualization of penetration of Decalin from the fracture into the saturated core with hexadecane . . . . . . . . . . . . 61

4.38 Variation of averaged CT numbers along the core in different time steps - Eicosane-Decalin system . . . . . . . . . . . 62

4.39 Variation of average of solvent saturation along the core in different time steps - Eicosane-Decalin system . . . . . . . . . 64

4.40 Recovery profile of Eicosane . . . . . . . . . . . . . . 65

4.41 Visualization of penetration of Decalin from the fracture into the saturated core with Eicosane . . . . . . . . . . . . 67

4.42 Averaged CT numbers along the core in different tims - HexadecanePentane system . . . . . . . . . . . . . . . . . . . 68

4.43 Variation of average of solvent saturation along the core in different time steps - Hexadecane-Pentane system . . . . . . 69

4.44 Recovery profile of Hexadecane . . . . . . . . . . . . . . . . . 70

4.45 Visualization of penetration of Pentane from the fracture into the saturated core with Hexadecane . . . . . . . . . . . . . 73

5.1 Geometry of the model . . . . . . . . . . . . . . . 75

5.2 R-Z cross-sectional view of MoReS simulation model . . . . . 76

5.3 History match of soak experiment with pentane and decalin $\mathrm{D}=9 \mathrm{E}-9 \mathrm{~m}^{2} / \mathrm{s} \ldots \ldots \ldots \ldots \ldots 78$

5.4 Simulation for the cases with and without gravitational forces for Pentane-Decalin system . . . . . . . . . . . . . . 79

5.5 Sensitivity analysis of the simulation with respect to the permability .................... . . 80

5.6 Predicted recovery profiles by varying the grid block sizes. Note that grid block size of $1 \mathrm{~mm}$ gives adequate results . . . 81 
5.7 History match of soak experiment with hexadecane and decalin $-\mathrm{D}=1 \mathrm{E}-11 \mathrm{~m}^{2} / \mathrm{s} \ldots \ldots \ldots . \ldots . \ldots . \ldots 8$

5.8 Simulation for the cases with and without gravitational forces for Hexadecane-Decalin system . . . . . . . . . . . . . . 84

5.9 History match of soak experiment with hexadecane and decalin $-\mathrm{D}=3 \mathrm{E}-11 \mathrm{~m}^{2} / \mathrm{s} \ldots \ldots \ldots \ldots$

B.1 The caption goes here . . . . . . . . . . . . . . . . . 102

B.2 Simplified system for image reconstruction with algebraic method 105

B.3 Illustration of coordinates for an object and its projection . . 106

B.4 The Image and its projection at specified angle and the related sinogram . . . . . . . . . . . . . . . 107

B.5 Schematic illustration of Fourier Slice Theorem and Radon Transform . . . . . . . . . . . . . . . . . 109 


\section{List of Tables}

3.1 Properties of experimental fluids . . . . . . . . . . . . . . . . 19

4.1 Comparison of solvent saturation using original matrices and the averaged matrix . . . . . . . . . . . . . 27

5.1 Gravity and Diffusion time and $\mathrm{N}_{\mathrm{GD}} \ldots \ldots$. . . . . . . . 87 
Chapter 1

\section{Introduction}




\begin{abstract}
Solvent injection has been considered as an efficient method for enhancing oil recovery from fractured reservoirs. If the mass transfer would be solely based on diffusion, oil recovery would be unacceptably slow. The success of this method therefore depends on the degree of enhancement of the mass exchange rate between the solvent residing in the fracture and the oil residing in the matrix.

A series of soak experiments have been conducted to investigate the mass transfer rate between the fracture and the matrix. In a soak experiment, a porous medium containing oil is immersed in an open space containing the solvent to simulate the matrix and the fracture respectively. We use a CT scanner to visualize the process. The experimental data are compared with a simulation model that takes diffusive and gravitational forces into account. We find that the initial stage of all experiments can be described by a diffusion-based model with an enhanced effective diffusion coefficient. In the second stage enhancement of the transfer rate occurs due to the natural convection of solvent in the fracture. The experiments are quantitatively modeled by numerical simulations. We find that transfer rates depend on the properties of the rock permeability, the viscosity and the density of solvent and oil. The gravity enhanced transfer is quantified by comparison of experimental and simulated results.
\end{abstract}




\section{$1.1 \quad$ Introduction}

Fractured reservoirs contain about $20 \%$ of the world oil reserves (Saidi, 1983; Firoozabadi, 2000). However, compared to the conventional (non-fractured) reservoirs, the recovery from these reservoirs can be low. This is caused by the permeability contrast between the fracture and matrix, due to which the flow rate in the fractures is orders of magnitude larger than the flow rate in the matrix; in the absence of efficient transfer between matrix and fracture the injected fluids bypass the oil in the matrix. In other words, economical recovery requires sufficient transfer from the matrix to the fracture. When the matrix is water-wet, and the matrix blocks have low permeabilities (in the $\mathrm{mD}$ range), water injection leading to capillary driven counter-current imbibition of water, can be effective (Firoozabadi, 2000; Salimi and Bruining, 2010). Many fractured reservoirs, however, are oil wet inhibiting capillarydriven counter-current imbibition and hence water injection may become inefficient. For example, the Ghaba North field in Oman is an extensivelyfractured oil-wet carbonate that has achieved only $20 \%$ recovery after more than 20 years of production (Al-Hadhrami and Blunt, 2001).

In such a case, gas injection may be a viable alternative because the larger density difference between the oil inside the matrix and the injected gas (compared to the density difference between water and oil) leads to larger gravity forces to oppose the capillary forces that hold the oil in place (Le Gallo et al., 1997). Such a process is called Gas Oil Gravity Drainage (GOGD). Under favourable conditions oil may spread on the omnipresent connate water in the presence of gas, leading to very low residual oil saturations. Gravity drainage is favourable for vertically long matrix blocks, high matrix permeability and low interfacial tensions. The negative effect of capillary hold up in gas-oil gravity drainage (GOGD) can be avoided, when gas is injected under miscible conditions. In the absence of capillary pressure no interface exists between miscible fluids of different compositions (Orr, 2007; Farzaneh et al., 2010). Therefore recovery in the fractured reservoirs can be improved by increasing the pressure or injecting appropriate fluids (miscible or near miscible) that result in reduction of capillary pressure and interfacial tension (Firoozabadi et al., 1997; Adibhatla and Mohanty, 2008; Karimaie and Torsaeter, 2008; Guevara-Torres et al., 2009; Ameri Ghasrodashti et al., 2012) Injection of a miscible gas improves the ultimate recovery, because the miscibility adds the advantage of single-phase flow and eliminates interfacial tension (Correa et 
al., 1990; Dindoruk and Firoozabadi, 1996; Verlaan and Boerrigter, 2006). In one-dimensional flow, recoveries close to $100 \%$ are observed for fully miscible fluids (Lewis et al., 2008; Ameri Ghasrodashti et al., 2012); this is the main reason why miscible processes are attractive EOR methods.

In most practical implementations of a GOGD process, the produced gas will be recycled, re-compressed and re-injected into the reservoir. When the reservoir is deep and the oil mainly consists of heavy components, the compression costs required to achieve miscibility in the reservoir become too high (Farajzadeh et al., 2010). For example $\mathrm{N}_{2}$ or $\mathrm{CO}_{2}$, which are currently used for EOR purposes, have only a limited range of applicability because of their phase behaviour; full miscibility is only achieved for lighter oils and at sufficiently high reservoir pressures (Berg et al., 2010).

The magnitude of oil recovery by solvent injection largely depends on the degree of mass exchange between the solvent in the fracture and the oil in the matrix. Initially the solvent in the fracture is transferred into the matrix by diffusion and mixes with the oil in the matrix. Mixing of the solvent and the oil affects the properties of the oil; for example, it reduces the oil viscosity, changes the volume of oil, and depending on the type of solvent it decreases or increases the oil density. An increase in oil density may also lead to Rayleigh-Taylor instabilities (Strutt, 1883; Taylor, 1949). The (naturally) convective flow accelerates the movement of solvent into the matrix and enhances the mixing process, which can result in accelerated oil production from the matrix. If the mass transfer of the solvent is governed solely by diffusion, the time required to produce the same amount of oil will be much larger, and is generally unacceptable from a practical point of view.

Investigation of the mechanisms underlying mass transfer between the solvent in the fracture and the oil in the matrix has been the subject of numerous studies (da Silva and Belery, 1989; Perez-Cardenas et al., 1990; Thiebot and Sakthikumar, 1991; Morel et al., 1993; Firoozabadi et al., 1997; Le Gallo et al., 1997; Darvish et al., 2006; Hatiboglu and Babadagli, 2008; Karimaie and Torsaeter, 2008; Trivedi and Babadagli, 2008; Er and Babadagli, 2010; Stalgorova and Babadagli, 2010). An overview of these investigations is given in the literature survey section below. However, in summary there is a lack of experimental data that fully visualize the process and its comparison with natural convection transport models 
Despite the great interest and significant research effort, there is still a need of more experimental data of the detailed mass transfer mechanisms. Visual experiments are able to provide direct evidence of the mass transfer mechanisms between the matrix and fracture for different oil-solvent viscosity and density ratios. Moreover, a detailed analysis of the transfer mechanisms via comparing the data with transport models is missing in the literature. Therefore, the scope of this paper is to analyze and visualize miscible displacement between fracture and matrix for different density and viscosity ratios. In order to visualize and quantify the process, a CT scanner has been used. X-ray tomography is a common technique for investigating the transport phenomena in three-dimensional porous media (Wellington and Vinegar, 1987; Withjack and Akervoll, 1988; Sorbie et al., 1989; Midtlyng and Stensen, 2000; Tang and Kovscek, 2005; Zitha and Uijttenhout, 2005; Zinati et al., 2007; Simjoo et al., 2009; Berg et al., 2010; Farajzadeh et al., 2010; Andrianov et al., 2011).

\section{$1.2 \quad$ Literature Survey}

There are only a few experimental studies in the literature that focus on the diffusion mechanism in naturally fractured reservoirs. Studies on the matrix-fracture interaction (diffusion- and convection-driven mass transfer) are limited in number compared to those on imbibition or drainage type of transfer process, especially from visualization point of view. The majority

of laboratory work on diffusion effects in naturally fractured reservoirs have been published after 1990. Before this period, simulation studies were conducted to investigate the diffusion mechanism on oil recovery in naturally fractured reservoirs.

\subsubsection{Laboratory studies on mass transfer between ma- trix and fracture}

Thiebot and Sakthikumar (1991) studied GOGD and mass transfer in cylindrical cores that were surrounded by fluids. This represented a fractured porous medium. They injected equilibrium gas from the top of a saturated core in the fracture, and oil was produced from the bottom. There was no mass transfer between the injected gas and displaced oil as the gas and oil 
were already in thermodynamic equilibrium. Therefore, gravity drainage was the main recovery mechanism. They continued the experiment by injecting methane and nitrogen, instead of equilibrium gas. They concluded that injection of a non-equilibrium gas leads to significant additional oil recovery even after gravity drainage equilibrium has been attained; this can be attributed to the mass transfer between the injected non-equilibrium gas, in this case nitrogen and methane, in the fracture and the live oil in the matrix.

Morel et al. (1993) conducted a one-dimensional experiment and threedimensional experiments to investigate the combined effect of diffusion and stripping in matrix blocks of light-oil fractured reservoirs subjected to methane or nitrogen gas flooding. In the 3-D configuration gas is surrounding the core. The study was performed with synthetic binary mixtures of methane and pentane. Experiments were conducted at $\mathrm{P}=10.1 \mathrm{MPa}$. They concluded that the effect of initial gas saturation on the oil recovery is insignificant. In their experiments pentane recovery was linear with time, which indicates that the recovery process is not governed by pure diffusion. Pentane recovery by methane injection was 1.6 times faster than that by nitrogen injection. During nitrogen injection, saturation profiles along the core revealed a strong capillary end effect. This effect resulted in accumulation of oil in the matrix near the fracture. It was also concluded that when nitrogen is the injection gas, flow rate has a small effect on pentane recovery. It should be noted that contrary to more realistic scenarios pentane is a single component and hence does not contain any dissolved gas, which usually makes the difference between nitrogen injection and methane injection less pronounced (Boersma and Hagoort, 1994) than intuitively expected (see, however, Morel et al. (1993)).

Darvish et al. (2006) conducted $\mathrm{CO}_{2}$ injection experiments at reservoir condition to study the component exchange between the matrix and the fracture system during the $\mathrm{CO}_{2}$ injection. A $2 \mathrm{~mm}$ gap between the core and core holder simulated a fracture in their experiments. Both fracture and core were completely saturated with oil in order to ensure a homogeneous fluid composition in the matrix. During the experiment $\mathrm{CO}_{2}$ was injected at a low and constant rate into the fracture system (from the top of the core) and oil was produced from the bottom. They studied the component exchange between the oil in the matrix and $\mathrm{CO}_{2}$ in the fracture by analyzing the produced fluids. Both simulation and experimental results showed the 
importance of the diffusion mechanism. The simulation results showed that the key mechanism to recover oil from a tight matrix block $(4 \mathrm{mD})$ is diffusion and that gravity drainage has no significant effect. They recommended that the existing compositional simulators should be modified by properly accounting for diffusive transport. Some recent research has been devoted to consider diffusion in numerical simulations (Hoteit and Firoozabadi, 2009).

In order to investigate an EOR process for oil-wet carbonate fractured reservoirs, Karimaie and Torsaeter (2008) conducted experiments of secondary and tertiary gas injection into oil-wet carbonate cores. The authors noted that diffusion plays an important role in both secondary and tertiary oil recovery. In their experiments injection of $\mathrm{CO}_{2}$ resulted in a higher recovery than equilibrium gas or nitrogen in tertiary recovery, because better dissolution and the ensuing IFT reduction contributed to oil recovery.

Berg et al. (2010) conducted an experimental study of miscible displacement of oils by a non-conventional solvent, carbon disulfide $\left(\mathrm{CS}_{2}\right)$ in a porous medium. In their experiments the model oil is displaced by $\mathrm{CS}_{2}$. This study does not consider a fractured medium. The reason to include this in the literature overview is that it concerned miscible displacement and CT-scanning measurements. They employed a CT scanner to visualize and quantify the process. The dimensionless scaling groups such as the Peclet number, gravity to viscous forces ratio $G$, and the logarithmic viscosity ratio $R$ were used to characterize flow regimes and parameterize the experimental data. The 3D spatial distribution of the solvent $\left(\mathrm{CS}_{2}\right)$ and the displaced oils showed that the dominating displacement patterns were channeling in high permeability streaks, viscous fingering of $\mathrm{CS}_{2}$ and gravity under-run of $\mathrm{CS}_{2}$ due to its relatively high density. They identified three distinct regimes: (a) viscous forces dominate at small gravity number $G$, followed by (b) a regime of intermediate $G$ where viscous and gravity forces are in balance followed by, and (c) a regime with large $G$ where gravity and diffusion are the dominating mechanisms.

Hatiboglu and Babadagli (2008), used 2D glass-bead models to study the co- and counter-current type of transfers due to diffusion and -convection caused by the buoyant forces between fracture and matrix. In their experiments the density and viscosity of the solvent (pentane) was lower than the density and viscosity of the oils. Moreover, only the bottom part of the 
porous medium was exposed to the solvent. Due to the lower viscosity and density of the solvent, they observed instabilities in their experiments. The experiments showed two stages: an initial stage dominated by diffusion leading to fingering and a later stage dominated by natural convection due to the density difference between the solvent and the oil.

Trivedi and Babadagli (2008) performed experiments to study the efficiency of a diffusion process between the matrix and fracture, while fluids were flowing in the fracture and found that although with high solvent injection rates initially more oil can be produced, they found that a low injection rate leads to higher ultimate oil recovery due to diffusion. In all experiments the density and viscosity of the solvent was lower than that of the oil in the matrix.

\subsubsection{Numerical studies on mass transfer between ma- trix and fracture}

Coats [1989] included the effect of diffusion in dual-porosity models. He solved the diffusion equation in one dimension to estimate diffusion transient time. The diffusion equation was derived for a linear horizontal core with length $l$ initially saturated with a fluid of unit concentration and then exposed to zero fluid concentration at $\mathrm{x}=0$ and $\mathrm{x}=l$.

Da Silva and Belery [1989] simulated the effect of diffusion on oil recovery in highly fractured reservoirs with low matrix permeability. He solved the diffusion equation analytically for a matrix block for a step change in concentration at the matrix boundary. Their analytical simulation results showed the significant effect of diffusion on oil recovery, especially for matrix blocks of small size, i.e., of the order of several feet or less. Because it is expected that the diffusion in a one foot grid block, for typical liquid-liquid diffusion coefficients, takes 3 years, they stressed the importance of taking into account the effect of diffusion on oil recovery in simulation of naturally fractured reservoirs.

Hu et al. [1991] simulated the experiments of Morel et al. They combined an analytical solution for mass transfer in the fracture with a numerical model 
in the core. They showed the importance of the diffusion mechanism in transporting $\mathrm{N}_{2}$ and $\mathrm{C}_{1}$ in the porous medium. They showed that diffusion is not the only mechanism that recovers oil from the matrix. They explained that as the fluid in the core contacts nitrogen, the mass of the oleic phase increases (based on the ternary phase diagram of methane-pentane-nitrogen). In other words, the oil would diffuse from the fracture into the matrix, which is impossible because the injected gas in the fracture does not contain oil. The only way to keep phases in equilibrium is to have oil supplied from the lower part of the core. This is the reason for oil convection from the matrix towards the fracture.

Hoteit and Firoozabadi [2009] simulated gas injection using the finite element method. They concluded that for a low permeability matrix ( 1 and $0.1 \mathrm{mD}$ ) the effect of diffusion is more important than what current models predict. Their simulation results showed $25 \%$ increase in oil recovery by including diffusion compared to the simulation without diffusion. They claimed the effect of diffusion was more pronounced for smaller fracture spacing.

The experiment conducted by Darvish et al. [2006] was simulated by Moorget et al. [2010] by the finite element method. They also found the importance of diffusion as an recovery mechanism but not as significant as the Darvish et al. [2006] experimental results.

\subsection{Objectives of the project}

The objectives of this thesis can be divided into four parts as follows,

1. To visualize the interaction between the solvent in the fracture and oil in the matrix.

2. To investigate the underlying oil production mechanism (diffusion, gravity induced convection)

3. To investigate the effects of different parameters such as density and viscosity of the fluids and permeability of the rock.

4. To obtain relevant parameters in order to history match the experiment and the numerical model. 
The structure of the report is as follows. In Chapter 2, the physical model and governing equations are discussed briefly. Chapter 3 describes the experimental procedure. The experimental and simulation results are shown in Chapter 4 and Chapter 5 respectively. Finally we end this report with some concluding remarks. Appendix A gives a brief theoretical background. and in Appendix B, a brief explanation about the image reconstruction in $\mathrm{X}$-ray computed tomography is provided. 


\section{Chapter 2}

\section{Formulation}

\section{Introduction}

We formulate conservation laws governing the invasion of solvent to a porous medium saturated by oil. This set of equations governs the miscible displacement. Recovery form an immersed core inside a solvent occurs in two stages: 1. solvent diffusion into the oil and 2. gravity induced flow to expel the mobilized oil out of the matrix block. Natural convection and diffusion are taken into account and density is considered variable by changing the concentration of component within the mixture. Then we make the final equations dimensionless for numerical solutions and the scaling groups are used to parametrize the experimental data.

\subsection{Physical Model}

In a soak experiment we consider the invasion of solvent from a fracture around the core into the matrix originally saturated with oil such that the oil is produced from the core. The oil and the solvent are miscible. If two miscible fluids are in contact, they will slowly diffuse on another. This diffusion arises because of random motion of the molecules, therefore the diffusive effects should be considered in mass balance equation. In the following, a set of governing equations and scaling groups for miscible displacement is derived. In this study the transport equation is derived using the volume fraction of each component which is a function of density of each component in the mixture, i.e. the variation of density is also taken into account. The 
experimental situation is described as miscible flow through a porous medium on the basis of Darcy's law. Because the solvent and the oil used in this study are fully miscible, always a single phase is formed with a varying composition.

In the soak experiment, in the absence of convective flow, there are two mechanisms which contribute to production from the core. These two mechanisms are:

1. Diffusion: the solvent is imbibed into the core by diffusion and reduce the oil viscosity.

2. Gravity-driven convection: as a result of viscosity reduction, oil mobility increases and due to density difference between the oil and the solvent, the oil is produced from the core.

Note that matrix diffusion is considered as a 1D process. This assumption is made based on faster migration of solvent in fractures than in the matrix, i.e. in the direction parallel to the fracture plane diffusive exchange is negligible compared to the direction perpendicular to the fracture plane.

The diffusion of solvent in the oil results in viscosity reduction of the oil. Consequently, the oil in the matrix is mobilized and is expelled out of the matrix by the gravity forces. Then gravity forces expel this mobilized oil out of the matrix. This creates spaces for the fresh solvent to flow into the matrix and keep the diffusion process continuing. In the absence of gravity forces, the only contribution mechanism is diffusion. However gravity forces result in faster movement of the solvent at the bottom of the sample than at the top (in case of denser solvent). This has an influence on the propagation of the front of the solvent in the core.

Assuming purely diffusive transport and ignoring the gravity-driven convection leads to an overestimation of the diffusion coefficient, whereas in reality gravity contribute to the transport. The gravity forces create an advancing shock front of solvent. For the same diffusion coefficient, the mass transfer is enhanced by the high concentration gradient across the shock front.

The vertical symmetry of the saturation profile along the core at early stage of experiment suggests that the displacement is governed by pure diffusion and not by gravity. Because in case of pure diffusion, solvent tends to 
enter the core from everywhere. In case of gravity takes over the mass transfer, depending on the density of the solvent, solvent penetrates either from the bottom or from the top of the core, i.e., if the solvent in the fracture is denser than the oil in the matrix the solvent tends to penetrate into the core from the bottom and if the oil in the matrix is denser than the solvent then the solvent penetrates from the top of the core. Consequently, the saturation profile becomes asymmetric.

The core has constant porosity of $\varphi$ and the permeability of $k$. For the fracture we assume a porosity equal to one, $\varphi_{f}=1$ and very high permeability.The core is placed vertically and the effect of gravity is taken into account.

The governing equations which are formulated in this study are:

- Conservation of Mass

- Darcy's law

- Transport Equation (Convection-Diffusion Equation)

Then the governing equations are made dimensionless for numerical solutions and the scaling groups are used to parametrize the experimental data. Peclet Number and Grashof Number are two important dimensionless numbers in this study. The Peclet number is an appropriate scaling group which can describe the problem. This number takes 'convective velocity' and 'diffusive velocity' into account. Darcys law provides an estimation for the convective velocity and the molecular diffusion.

\subsection{Governing Equations}

For a 2-D porous medium the governing equations for the described phenomena can be written as 


\subsubsection{Continuity Equation}

The mass conservation of the two components, the solvent and the oil, in our system is given by the following equations:

$$
\left\{\begin{array}{l}
\varphi \frac{\partial}{\partial t} \psi_{o}+\nabla\left(\psi_{o} u_{o}\right)=0 \\
\varphi \frac{\partial}{\partial t} \psi_{s}+\nabla\left(\psi_{s} u_{s}\right)=0
\end{array}\right.
$$

where $\psi_{o}$ and $\psi_{s}$ are oil and solvent volume fraction respectively and interpreted as the ratios of the densities of the component in the mixture, $\rho_{s}$ and $\rho_{o}$, and the pure components densities, $\rho_{S}$ and $\rho_{O}$,

$$
\left\{\begin{array}{l}
\psi_{o}=\frac{\rho_{o}}{\rho_{O}} \\
\psi_{s}=\frac{\rho_{s}}{\rho_{S}}
\end{array}\right.
$$

By the assumption that the mixture of the solvent and the oil behave as an ideal fluid with zero heat of mixing, there will be no volume change due to mixing and we can write the following equation:

$$
\frac{\rho_{s}}{\rho_{S}}+\frac{\rho_{o}}{\rho_{O}}=1
$$

where ' $s$ ' and ' $o$ ' denote the density of the solvent and the oil in the mixture and ' $S$ ' and ' $O$ ' denote the pure component density respectively. Note the sum of the volume fraction of each component is equal to one,

$$
\psi_{o}+\psi_{s}=1
$$

From Eq. 2.1 we obtain,

$$
\operatorname{div}\left(\psi_{\mathbf{o}} \mathbf{u}_{\mathbf{o}}+\psi_{\mathbf{s}} \mathbf{u}_{\mathbf{s}}\right)=\mathbf{0}
$$

or,

$$
\operatorname{div}\left(\mathbf{u}_{\mathbf{f}}\right)=\mathbf{0}
$$

where $u_{f}$ is the Darcy velocity of the mixture. 


\subsubsection{Darcy's Law}

Darcy's law reads

$$
u_{f}=-\frac{k}{\mu_{f}}\left(\nabla p+\rho_{f} g \mathbf{e}_{\mathbf{z}}\right)
$$

where the $\mu_{f}$ is the viscosity of the mixture, which can be calculated (for example) the quarter-power rule

$$
\mu_{f}=\left(\psi_{o} \mu_{o}^{-\frac{1}{4}}+\psi_{s} \mu_{s}^{-\frac{1}{4}}\right)^{-4}
$$

or from a log-linear viscosity mixing,

$$
\mu_{\text {mix }}=\mu_{\text {model oil }}^{\mathrm{C}_{\text {model }}} \times \mu_{\text {solvent }}^{\mathrm{C}_{\text {solvent }}}
$$

The density is given by

$$
\rho_{f}=\rho_{s}+\rho_{o}
$$

\subsubsection{Transport Equation}

The transport equation can be expressed as,

$$
\left\{\begin{array}{l}
\varphi \frac{\partial}{\partial t} \psi_{o}+\nabla\left(\psi_{o} u_{f}\right)=\varphi D \nabla^{2} \psi_{o} \\
\varphi \frac{\partial}{\partial t} \psi_{s}+\nabla\left(\psi_{s} u_{f}\right)=\varphi D \nabla^{2} \psi_{s}
\end{array}\right.
$$

where $\psi$ is the volume fraction of the component and $D$ is the diffusion coeficient.

\subsection{Dimensionless form of the equations}

We modify Eq. 2.11 to put it in the dimensionless form

$$
\varphi \frac{1}{t_{R}} \partial t_{D} \psi_{o}+\frac{u_{R}}{x_{R}} \nabla_{D} \cdot\left(\psi_{o} u_{f D}\right)=\frac{D}{x_{R}^{2}} \varphi \nabla_{D}^{2} \psi_{o}
$$

and the same equation for the solvent volume fraction $\psi_{s}$ 


$$
\frac{x_{R}^{2}}{D t_{R}} \partial t_{D} \psi_{o}+\frac{u_{R} x_{R}}{\varphi D} \nabla_{D}\left(\psi_{o} u_{f D}\right)=\nabla_{D}^{2} \psi_{o}
$$

We define the reference time $t_{R}=x_{R}^{2} / D$ and use the Peclet number

$$
P e=\frac{u_{R} x_{R}}{\varphi D}
$$

to obtain

$$
\partial t_{D} \psi_{o}+P e \nabla_{D}\left(\psi_{o} u_{f D}\right)=\nabla_{D}^{2} \psi_{o}
$$

Peclet number can be expressed as the ratio of convective velocity and and the diffusive velocity:

$$
P e=\frac{\text { Convective Velocity }}{\text { Diffusive Velocity }}
$$

Now we turn to Darcy's law.

$$
u_{f D}=\frac{k_{D}}{\mu_{f D}} \frac{k_{R} p_{R}}{u_{R} \mu_{R} x_{R}}\left(\nabla_{D} p_{D}+\frac{\rho_{R} x_{R}}{p_{R}} \rho_{f D} g \mathbf{e}_{\mathbf{z}}\right)
$$

It is convenient to choose $p_{R}$ as follows

$$
p_{R}=\frac{u_{R} \mu_{R} x_{R}}{k}
$$

Hence we find

$$
u_{f D}=\frac{k_{D}}{\mu_{f D}}\left(\nabla_{D} p_{D}+\frac{k_{R} \rho_{R} g}{u_{R} \mu_{R}} \rho_{f D} \mathbf{e}_{\mathbf{z}}\right)
$$

There is no characteristic velocity in the system and hence we construct a reference velocity based on the parameters that we have. We use the following expression for $u_{R}$

$$
u_{R}=\frac{\mu_{R}}{\rho_{R} x_{R}}:=\frac{\nu_{R}}{x_{R}}
$$

and after substitution in eq. 2.18 we find

$$
u_{f D}=\frac{k_{D}}{\mu_{f D}}\left(\nabla_{D} p_{D}+\frac{k_{R} x_{R} g}{\nu_{R}^{2}} \rho_{f D} \mathbf{e}_{\mathbf{z}}\right)
$$


The term $\frac{k_{R} x_{R} g}{\nu_{R}^{2}}$ is also called the Grashof number $G r$.

$$
G r=\frac{k_{R} x_{R} g}{\nu_{R}^{2}}
$$

Therefore we obtain

$$
u_{f D}=\frac{k_{D}}{\mu_{f D}}\left(\nabla_{D} p_{D}+G r \rho_{f D} \mathbf{e}_{\mathbf{z}}\right)
$$

The dimensionless equation for incompressible fluids can be written as

$$
\operatorname{div} \mathbf{u}_{f D}=0
$$

which can be verified by adding the solvent equation to the oil equation. Hence for numerical modeling, following dimensionless governing equations should be solved numerically with a numerical method,

1. Conservation of Mass

$$
\operatorname{div} \mathbf{u}_{f D}=0
$$

2. Darcy's Law

$$
u_{f D}=\frac{k_{D}}{\mu_{f D}}\left(\nabla_{D} p_{D}+G r \rho_{f D} \mathbf{e}_{\mathbf{z}}\right)
$$

3. Transport Equation

$$
\frac{\partial \psi_{o}}{\partial t_{D}}+P e \nabla_{D}\left(\psi_{o} u_{f D}\right)=\nabla_{D}^{2} \psi_{o}
$$




\section{Chapter 3}

\section{Soak Experiment: Principles and Procedure}

\section{Introduction}

The aim of the experiment is to study in detail the transfer mechanism between the fracture and the matrix. The fracture is flooded by a solvent and the matrix contains the oil to be recovered. The experimental conditions are chosen as simple as possible, i.e., atmospheric pressures and ambient temperatures. We take a core plug filled with a model oil surrounded by solvent. Detailed observations are to be obtained with a CT-scanner. The experiment shows the penetration of the solvent in the matrix. The displacement mechanism during the experiment can be described as follows. During the experiment, the solvent penetrates into the core due to gravity forces and due to diffusion. Our aim is (a) to visualize the invasion of solvent using the CT-scanner, (b) to convert the CT data to values for solvent concentration in the core to calculate the oil recovery at different time steps and (c) to use the experimental data to elucidate the underlying oil recovery mechanisms. In all experiments, we assumed that the penetration of the solvent is radially symmetric. 


\subsection{Soak Experiment: Principles}

In a so-called soak experiment a core is placed inside a glass jar that has a spacer at the bottom. The core is fully saturated with oil. The space between the core and the wall of the jar, which mimics the fracture, is filled with solvent. Figure 3.1 depicts a schematic drawing of the experimental set-up, which is placed inside the CT-scanner.

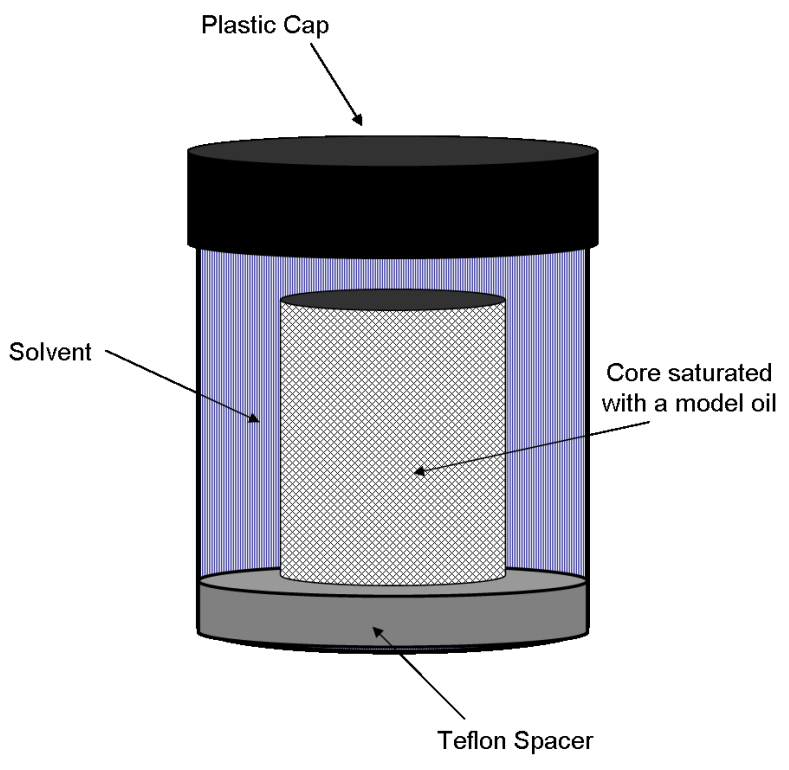

Figure 3.1: Schematic drawing of the glass jar containing the core in the experimental set-up

The spacer at the bottom of glass is used for two main reasons:

1. To allow interaction between the solvent and the oil from the bottom of the core sample

2. To keep the sample vertically in the glass jar. This is necessary for the correct calculation of saturations from CT images

All experiments are conducted with Bentheimer sandstone cores. At the beginning of each experiment, the core is completely saturated with oil, i.e., no initial water and trapped air is present. A doping agent "Iododecane" 
is added to the solvent at a concentration of $10 \mathrm{v} / \mathrm{v} \%$ to increase the CT contrast. Then the jar is filled with the doped solvent and placed inside the CT-scanner. After adjusting the setting of the CT-scanner, the oil saturated core is immersed in the solvent and left to soak for several hours. When the core is placed in the jar the first scan is taken immediately. This first CT scan is then used as a reference for the later concentration or saturation calculations.

A detailed description of the core saturation process is provided in Appendix C.

\subsection{Fluids and Experimental Conditions}

In soak experiments a saturated core is placed inside a glass jar. A Teflon spacer was employed at the bottom of the glass jar to allow interaction between the solvent and the oil at the bottom of the core as well as the top. This also helps keeping the sample in vertical position in the glass jar. The empty space between the core and the wall of the glass jar mimics a fracture. Experiments were conducted with sandstone core plugs of length $l=60$ $\mathrm{mm}$ and diameter $d=37.8 \mathrm{~mm}$. For each experiment we used a different core plug, although they were all drilled from the same rock block. Two different core plugs with high and low permeability were used. The porosity

of both sandstone cores were approximately 0.21 , giving a pore volume of $14.1 \mathrm{~cm}^{3}$. The permeability of Bentheimer sandstone core (high permeable) varied between 1200 and $1500 \mathrm{mD}$. All experiments were conducted at room temperature $\left(20^{\circ} \mathrm{C}\right)$ and atmospheric pressure. The volume of the solvent around the core in the glass jar was $258 \mathrm{~cm}^{3}$. In each experiment we employed different solvents and model oils.

1. Pentane/Decalin

2. Eicosane/Decalin

3. Hexadecane/Decalin

4. Hexadecane/Pentane

The properties of these fluids are listed in Table 3.1, the data are provided at standard conditions $\left(20^{\circ} \mathrm{C}\right.$ and atmospheric pressure) [SpringerMaterials]. 
Table 3.1: Properties of experimental fluids

\begin{tabular}{ccccc}
\hline \hline Material & Chemical Formula & Type & Density $[\mathrm{g} / \mathrm{cm} 3]$ & Viscosity [cP] \\
\hline \hline & & & \\
Decalin & $\mathrm{C}_{10} \mathrm{H}_{18}$ & Solvent & 0.896 & 3.355 \\
Pentane & $\mathrm{C}_{5} \mathrm{H}_{12}$ & Model oil/Solvent & 0.626 & 0.240 \\
Hexadecane & $\mathrm{C}_{16} \mathrm{H}_{34}$ & Model oil & 0.769 & 3.507 \\
Eicosane & $\mathrm{C}_{20} \mathrm{H}_{42}$ & Model oil & 0.789 & $4.01\left(36^{\circ} \mathrm{C}\right)$ \\
Iododecane & $\mathrm{C}_{10} \mathrm{H}_{21} \mathrm{I}$ & Doping agent & 1.250 & n.a \\
\hline
\end{tabular}

\subsection{Data Acquisition and Analysis}

Data analysis is based on CT images. CT scans are taken very frequently to record the density changes with time. Depending on the oil and the solvent the subsequent images are taken at different time intervals. At the beginning of the experiment the time intervals are small and gradually the time intervals increase as the rate of change decreases.

The output of the CT-scanner for each time step is four images from four different detectors. Each image has $512 \times 512$ pixels. These images can be converted to a $512 \times 512$ matrix containing the CT-numbers for each pixel using MATLAB. CT-numbers represent the changes in the density such that one Hounsfield unit represents $0.1 \%$ change in the density with respect to the calibration density scale. Further analyses are based on these CT-numbers matrix. CT-numbers can be converted to the solvent saturation using Eq.3.1 [Farajzadeh et al. 2009]. By assuming conservation of fluid volume, the amount of produced oil at each time step is equal to the saturation of the solvent in the core. Therefore Eq.3.1 can also be used for calculating the oil recovery.

$$
\% \text { Solvent }=\% \text { Produced Oil }=\frac{\mathrm{CT}_{\text {exp }}-\mathrm{CT}_{\text {oil }}}{\mathrm{CT}_{\text {solvent }}-\mathrm{CT}_{\text {oil }}}
$$

where $\mathrm{CT}_{\text {oil }}$ is the CT-number of the fully saturated core with oil, which can be obtained from the first scan. $\mathrm{CT}_{\text {solvent }}$ is the $\mathrm{CT}$-number of the fully saturated core with solvent. For constructing the CT-numbers of the fully saturated core with solvent it is assumed that a tiny region at the bottom of the core in the last time step is fully saturated by the solvent, then same 
region is taken from first time step in order to determine solvent/oil CTnumbers ratio. Further on this ratio and CT-numbers of $100 \%$ saturated core with oil are used to construct the CT-number of $100 \%$ saturated core with solvent for the entire core.

For the sake of having a check point, the last point of the recovery profile is determined by bulk data (fluid mixture outside the plug). By determining the CT-number of different composition of solvent and oil we can construct a calibration curve. This calibration curve can be used to determine the composition of fluid mixture outside the plug at each time step. The result is compared with the saturation that has been obtained from the CT-numbers in the matrix.

Different MATLAB routines were written to visualize and to quantify the process. For visualizing the process, the data are processed further by cropping the core from the entire image and changing the contrast. The details of the calculation process are provided in Chapter 4.

Note that before conducting the main experiment we want to do a bulk measurement with the substances. These measurements are done for calibration purposes. A brief explanation about image reconstruction in CT-scanner is provided in Appendix B.

The procedure of data analysis for visualizing the process and also determining the oil recovery is summarized as follows,

1. The CT-numbers of the four images are averaged at each time step.

2. The core is cropped from the surrounding environment.

3. The entire core is subdivided into the small parts (e.g. $3 \mathrm{~mm}$ height parts).

4. The arithmetic horizontal average of CT-numbers of each part is determined. Consequently, a "n $\times 1$ " matrix is produced. This matrix includes an averaged CT-number for ' $n$ ' divisions of the core.

5. The averaged CT-numbers are plotted versus the vertical position of the core for each time step. This plot help us to observe the changes of 
CT-numbers along the core during the experiment, i.e., the variation of oil density due to penetration of the solvent to the core can be observed. This plot also shows how the solvent penetrates into the core during the experiment.

6. The $\mathrm{CT}_{\text {Solvent }}$ is determined for horizontally averaged saturation calculations.

7. The horizontally averaged saturation of the solvent inside the core at each time step is calculated.

8. The recovery profile of the oil is determined.

9. A calibration curve is produced using the CT images of different compositions of the solvent and the oil in the absence of a core.

10. Using this calibration curves and the CT-numbers outside the core in the last time step we are able to calculate the amount of produced oil at the end of the experiment, i.e. the oil which was originally in the core and is now in the bulk.

11. The last point saturation obtained from the bulk is compared with the last value of the recovery profile obtained in [8].

12. The process at different time steps is visualized. 


\section{Chapter 4}

\section{Experimental Results}

In this chapter first an overall description about the data analysis procedure is given; subsequently the experimental results for each solvent/oil system are shown. For data analysis we follow the procedure, which is provided in Chapter 3. Note that before conducting the main experiments, several bulk measurements were done by the CT-scanner in order to calibrate the CT-scanner.

\subsection{Averaging the four slices for each time step}

The output of the CT scanner for each time step is four images. These images were taken from the middle of the core. The thickness of each slice is $0.5 \mathrm{~mm}$. At the first step of the data analysis, the average of these four images are determined. Arithmetic averaging of four matrices results in one matrix that can be used for further analysis. Figure 4.1 illustrates an example of the four CT-images (top four images) and the averaged image (bottom image) for one of the experiments. 

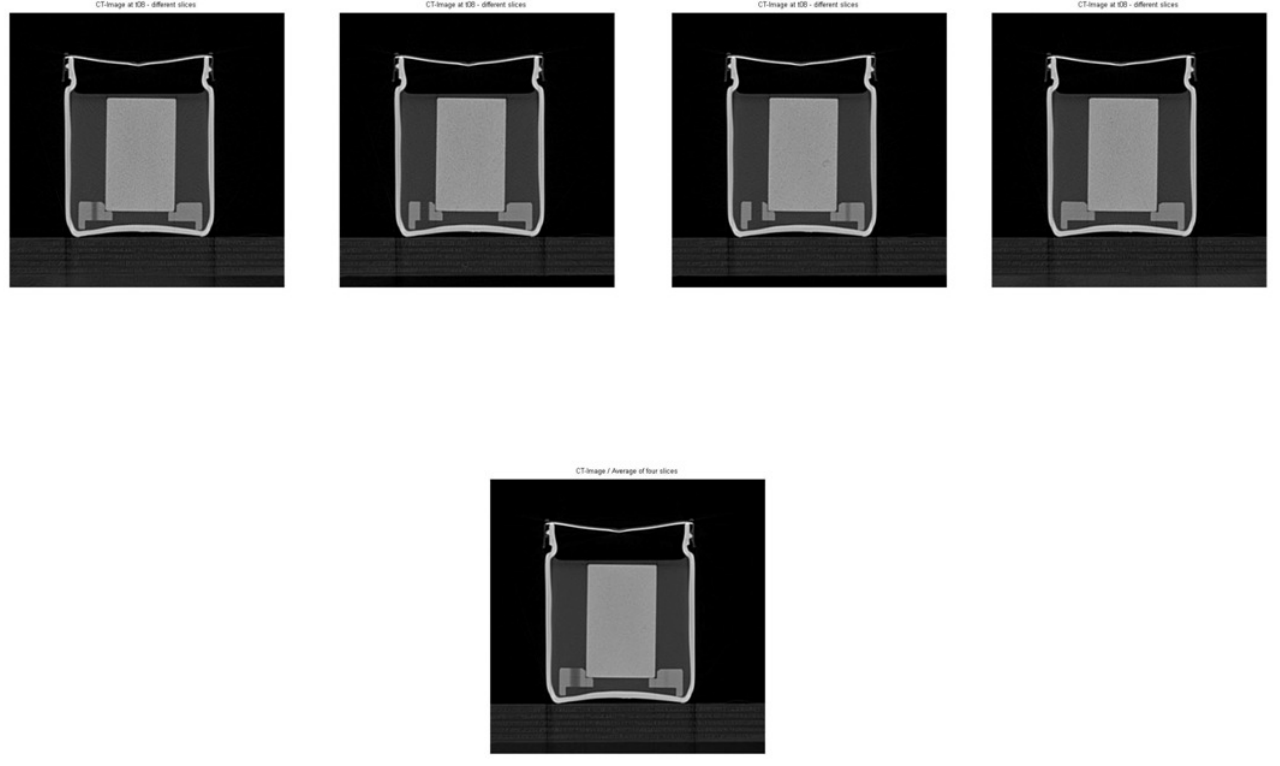

Figure 4.1: Four different CT-images from the middle of the core (top) and their averaged image (bottom)

The CT-numbers of the four slices in the three different horizons (top, middle and bottom of the core) are plotted together in Figure 4.2. 

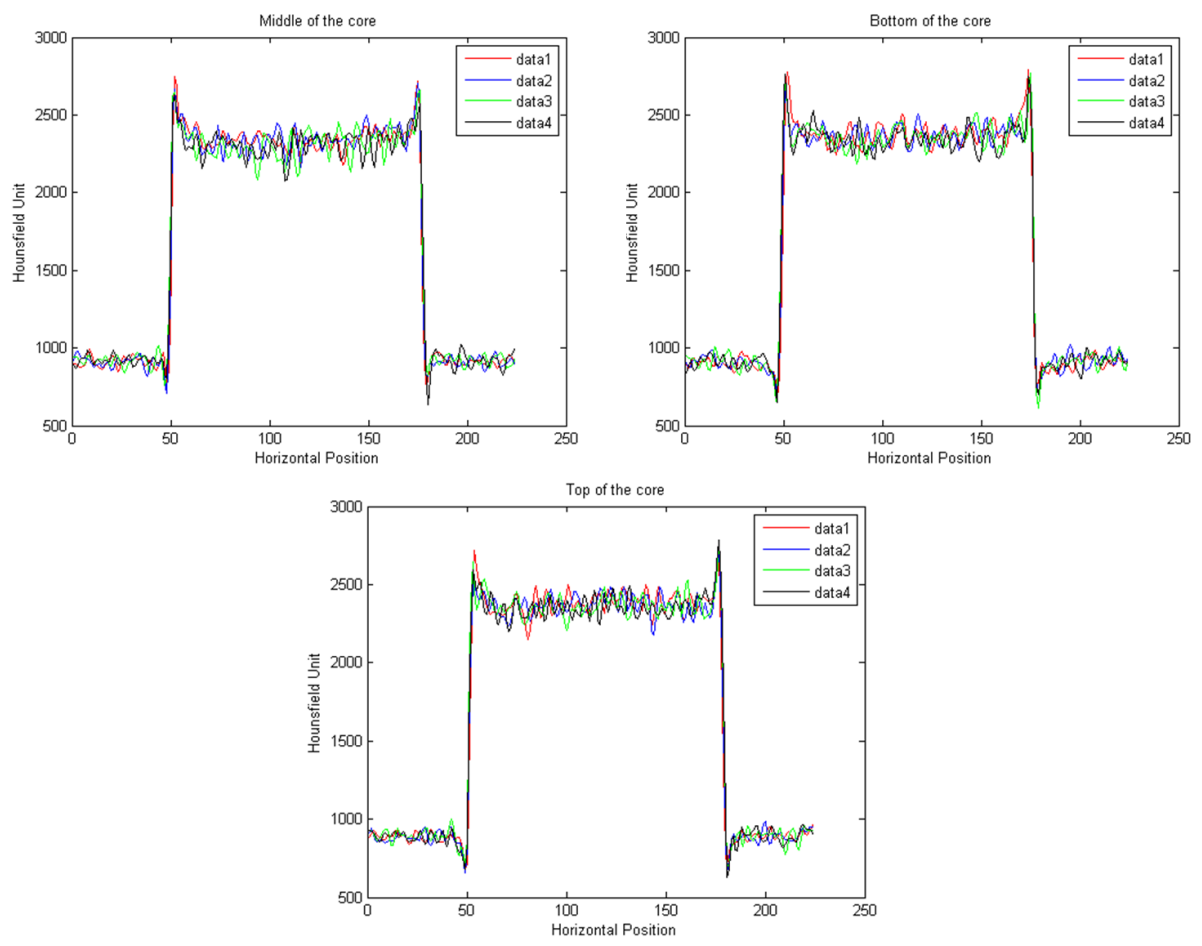

Figure 4.2: Comparison of CT-numbers in three different positions along the core in a same time step

In Figure 4.2 the horizontal axis and vertical axis represent the horizontal pixel number and the corresponding CT-numbers respectively. As implied in these plots, lower CT-numbers in the plot represent solvent around the core and higher CT-numbers are for the saturated core. The peak in the plot is due to an artifact at the edges of the core, where the X-ray penetrates the core and the density of the environment suddenly changes. 
As mentioned, the arithmetic average of these four images is taken and used for the further analysis. Figure 4.3 illustrates the averaged CT-numbers for all the pixels along the core and fracture.

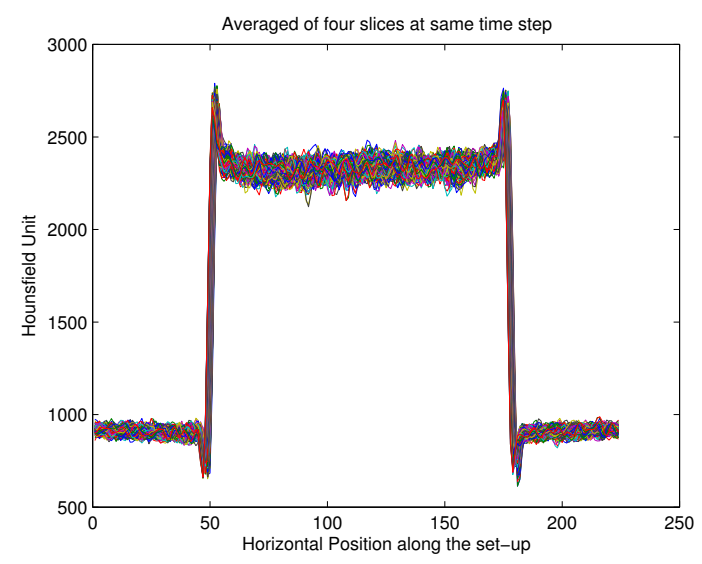

Figure 4.3: Average CT numbers of the core and the surrounding fracture

The image generated from this matrix is shown in figure 4.4. The dark margin in Figure 4.4 represents the solvent around the core and light rectangle shows the core.

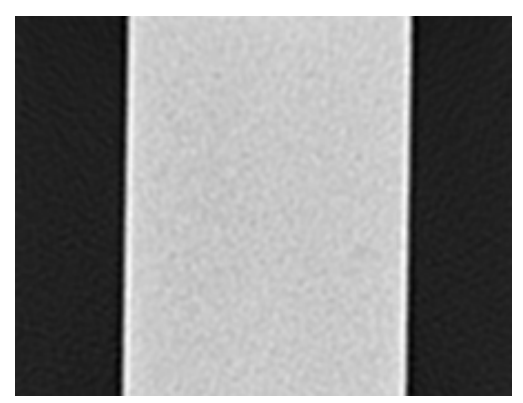

Figure 4.4: Average image of the core and the surrounding fracture

Comparison of the average of the four images and one of the images, which is measured by the CT scan, can give an idea about the discrepancy between the averaged $\mathrm{CT}$ numbers and the original CT numbers. 

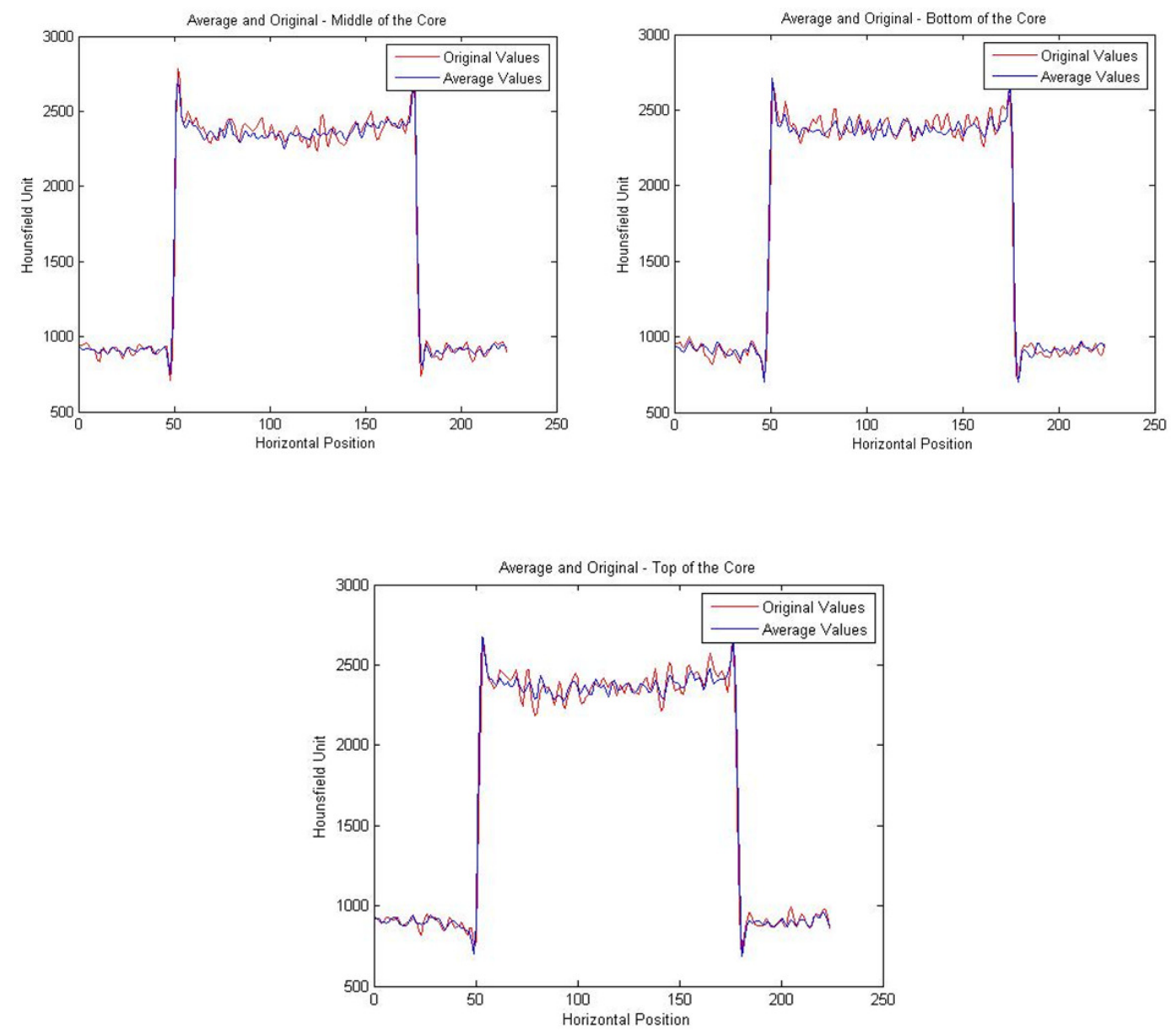

Figure 4.5: Comparison of the averaged CT numbers and the original CT numbers in different horizons along the core

To obtain an impression of the variation, we determine the solvent saturation obtained from average image and from the original images using Eq. 3.1. Table 4.1 shows these results. Afterwards the standard deviation and the mean of these data are calculated.

The standard deviation and the mean of data presented in Table 4.1 are:

$$
\sigma=0.05, \mu=0.48
$$

It could be seen that the value of the saturation obtained from the average matrix is located in the $\mu \pm \sigma$ range. 
Table 4.1: Comparison of solvent saturation using original matrices and the averaged matrix

\begin{tabular}{c||c}
\hline \hline Matrix & Solvent saturation \\
\hline \hline & \\
Image 1 & $53 \%$ \\
Image 2 & $50 \%$ \\
Image 3 & $47 \%$ \\
Image 4 & $40 \%$ \\
Averaged Image & $48 \%$ \\
\hline \hline
\end{tabular}

Up to here the first two steps of the analyzing procedure have been done (see Chapter 3). In the next sections we show the results and different aspects of the data analysis for different systems described in Chapter 3. For each system, first we study the variation of the CT numbers at different horizons along the core. Then we compare the CT numbers at different positions in different time steps. For the next step the core is subdivided into the $3 \mathrm{~mm}$ sections and the average of the CT number in each section is determined. In this way we can investigate how the process progresses with the time. Next, the saturation of the solvent and oil recovery in each time step is determined. Finally we end up with the visualization of the process.

\subsection{Pentane-Decalin}

In the first study, we conducted an experiment with Decalin in the space surrounding the core plug (represents the fracture) and Pentane in the core plug as model oil. The fluid properties are provided in Table 3.1 in Chapter 3 of this report. The results of this experiment is provided as follows.

\subsubsection{CT numbers at different horizons along the core}

When the solvent starts diffusing into the matrix, the composition of the fluid inside the core changes due to mixing of the oil and the solvent during the process. Depending on the sign of the density difference between oil and solvent, the invasion takes place from the bottom or top of the core. The invasion of the solvent into the core results in changing the density of the 
fluid in the core. In order to investigate how the solvent penetrates from the fracture into the core, CT-numbers can be plotted in different horizons (top, middle and bottom) at same time step. The position of the top, middle and bottom of the core is shown schematically in the figure 4.6,

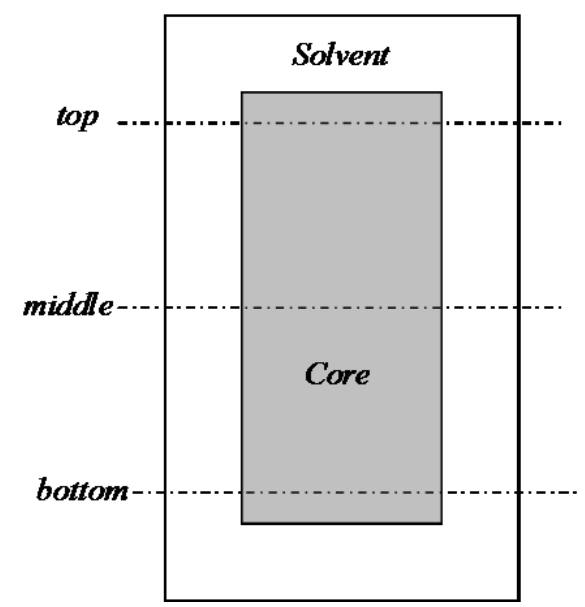

Figure 4.6: Schematic drawing of position of different horizons along the core

Figure 4.7 illustrates the variation of the CT-numbers at three different horizons: top, middle and bottom of the core 23 minutes after the start of the experiment. 


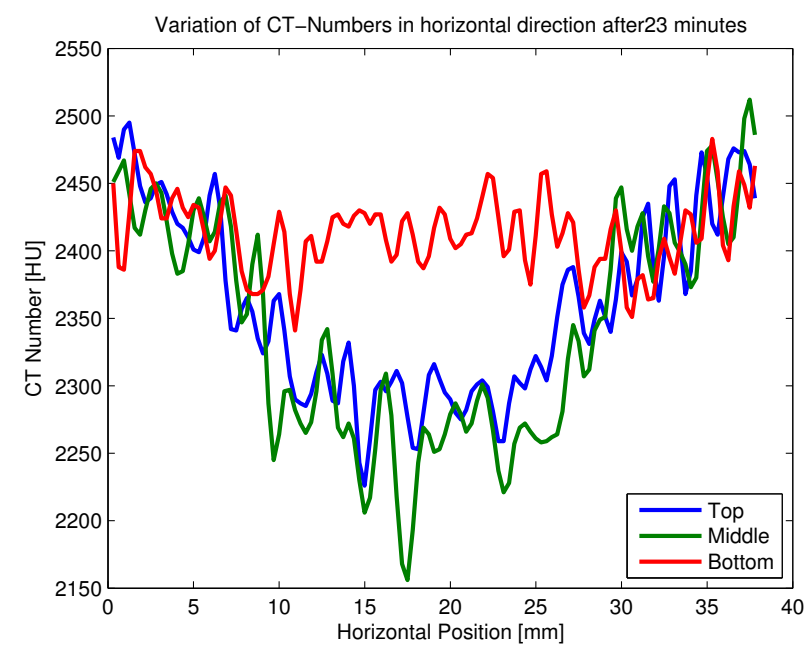

Figure 4.7: CT-numbers in three different horizontal position 23 minutes after the start of the experiment

Figure 4.7 shows that the CT-numbers at the bottom of the core are significantly higher than at the top and middle of the core. Higher CT numbers at the bottom of the core indicates that the solvent penetrates into the core predominantly from the bottom. The CT-numbers at top of the core are slightly higher than in the middle of the core, which means that the solvent penetrates into the core slowly. On the other hand, the CT-numbers at the side of the core for all three horizons are almost the same, which means that the solvent penetrates into the core from the sides equally.

In addition to analyzing the CT-numbers in three different horizontal crosssections, we can study the differences of the CT numbers in three different vertical positions: right, middle and left side of the core. In this way we can investigate how the solvent penetrates into the core from the sides. The position of the left, middle and right side of the core is shown schematically in Figure 4.8, 


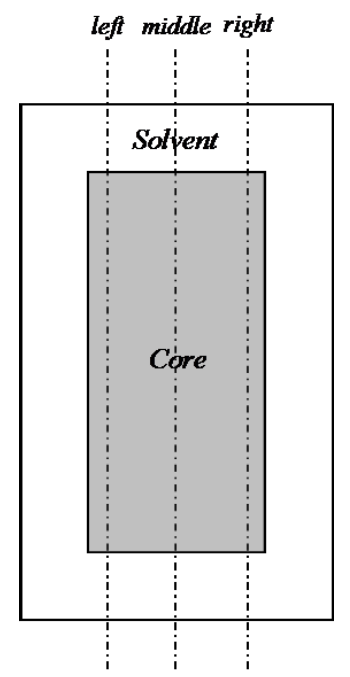

Figure 4.8: Schematic drawing of position of different horizons along the core

In Figure 4.9 the CT-numbers in vertical direction are plotted for different positions along the core 23 minutes after the start of the experiment.

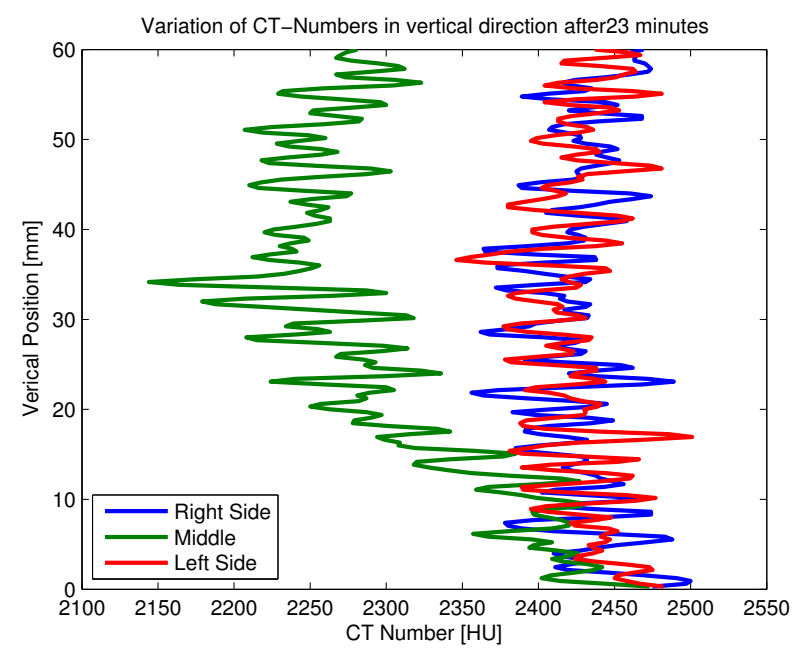

Figure 4.9: CT-numbers in three different vertical position 23 minutes after the start of the experiment 
It can be seen from Figure 4.9 that the CT-numbers in the right side and the left side of the core are almost the same and are higher than in the middle. This means that the solvent has invaded the core from the sides of the core as well as from the bottom. Note that this observation confirms the combined effect of diffusion and gravitational forces. At the later times this plot would be as shown in Figure 4.10 (after 21 hours). In Figure 4.10 we can see that the CT-numbers for the right side, left side and the middle are almost the same, which means that the solvent has invaded up to the center of the core.

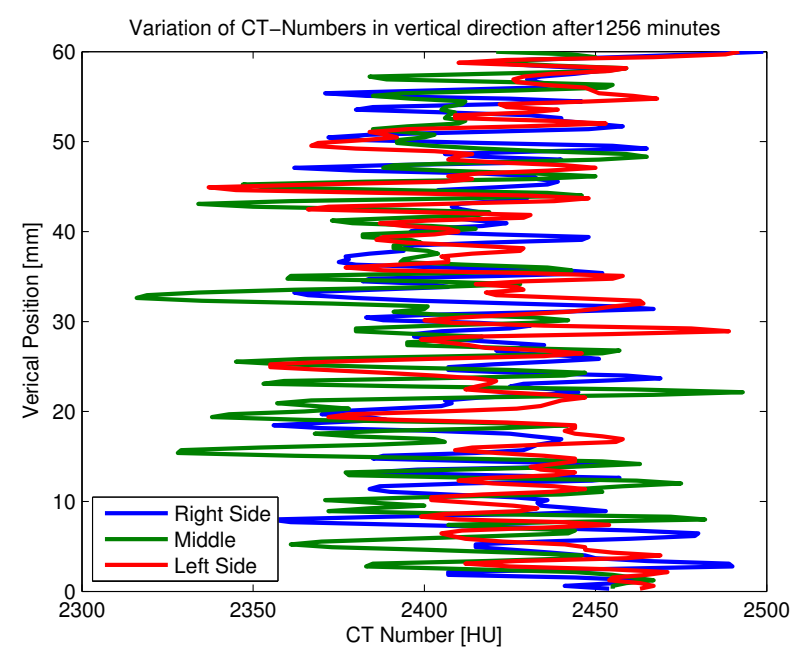

Figure 4.10: CT-numbers in three different vertical position 1256 minutes after the start of the experiment

\subsubsection{Comparison of CT numbers in horizontal and vertical sections in different times}

The evolution of the process can be investigated by comparing the CTnumbers in different time steps. As the solvent diffuses into the core, it mixes with the fluid inside the core. This mixing results in an increase of the fluid density inside the core. Therefore, the CT-numbers will increase inside the core because an increased solvent concentrations increases the density. Consequently, the variation of the $\mathrm{CT}$ numbers along the core is a tool to understand how the solvent penetrates into the core. The first step in our 
data analysis is to study the variation of CT numbers in different horizontal and vertical cross-sections of the core at different times. In each plot, the CT numbers at the initial time is also shown for the sake of comparison.

The positions of the top, middle and bottom horizons of the core and also the right, middle and left horizons of the core are shown schematically in figure 4.6 and figure 4.8 respectively.

\section{Top of the core}

Figure 4.11 illustrates the difference between the CT-numbers at the top of the core at five different times.

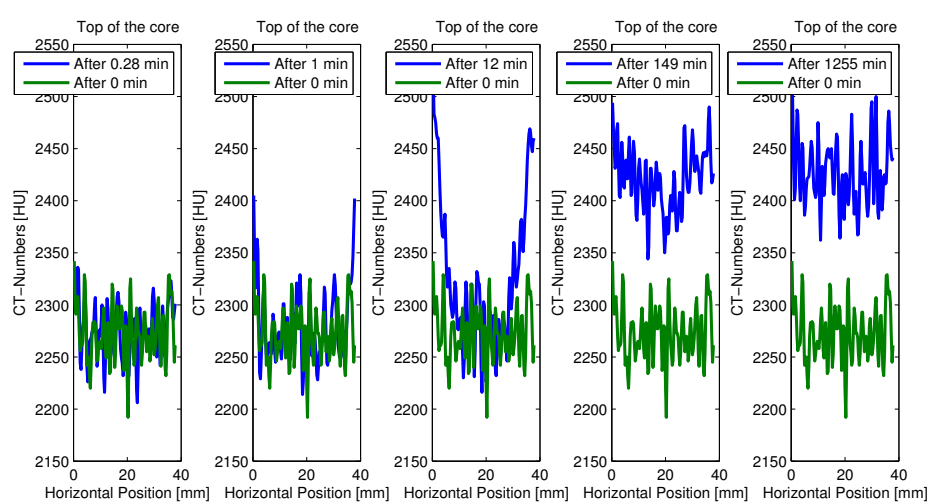

Figure 4.11: CT numbers at top of the core in different time steps. Green represents initial condition, which has been shown for each plot. Blue represents the current condition. 
As shown in Figure 4.11, the CT numbers at the top of the core change with time, which is a sign of solvent penetration into the core. It is obvious from this figure that the CT numbers at top of the core slowly increase from the sides. In other words, the solvent slowly penetrates the core from top of the core.

\section{Middle of the core}

Figure 4.12 illustrates the variation of CT numbers at the middle of the core for five different times.

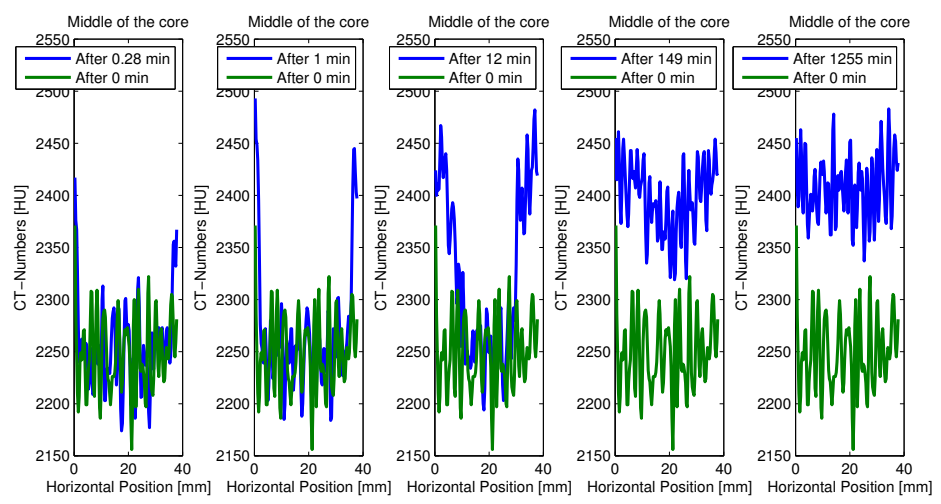

Figure 4.12: CT numbers at middle of the core in different times. Green represents initial condition, which has been shown for each plot. Blue represents the current condition.

In the middle section of the core, the solvent penetrates into the core from the sides and slowly it reaches up to middle of the core.

\section{Bottom of the core}

Figure 4.13 illustrates the CT numbers at bottom of the core for five different times. 

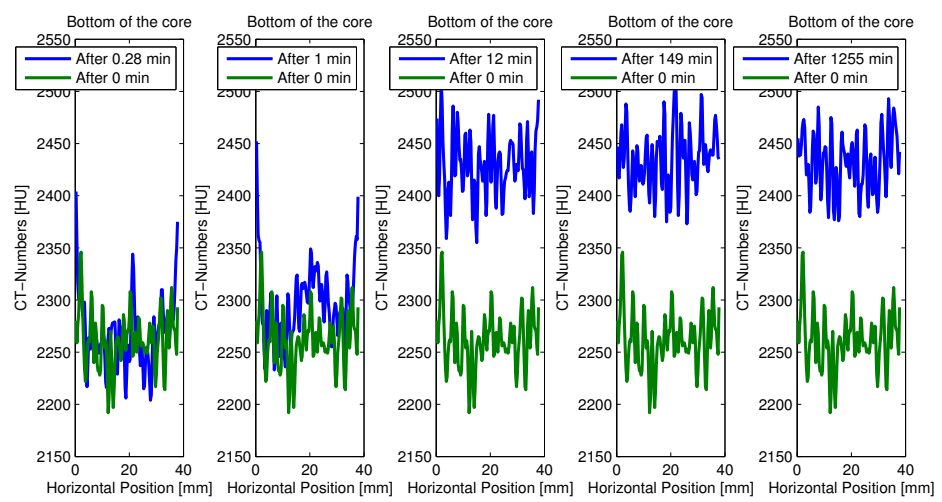

Figure 4.13: Variation of CT numbers at bottom of the core in different times. Green represents initial condition, which has been shown for each plot. Blue represents the current condition.

A Fast increase of the CT numbers at the bottom horizon shows that the rate of the solvent penetration into the core is high.

The following matters can be interpreted from comparison of the CT numbers in different positions:

- The CT numbers increase faster at the bottom of the core compared to the middle and the top of the core, which means that the solvent invades the core largely from the bottom.

- In the middle and the top of the core the CT numbers changes more from the sides, i.e., in these positions the solvent penetrates into the core largely from the sides and gradually reaches the middle of the core.

- CT numbers at the top of the core increase faster than in the middle horizon of the core. This indicates that the solvent penetrates into the core also from the top side of the core.

For a better understanding of the underlying mechanisms, we can study the variation of the $\mathrm{CT}$ numbers in vertical sections of the core at the same time step.

\section{Left side of the core}

Figure 4.14 illustrates the CT numbers at left side of the core for five different times. 


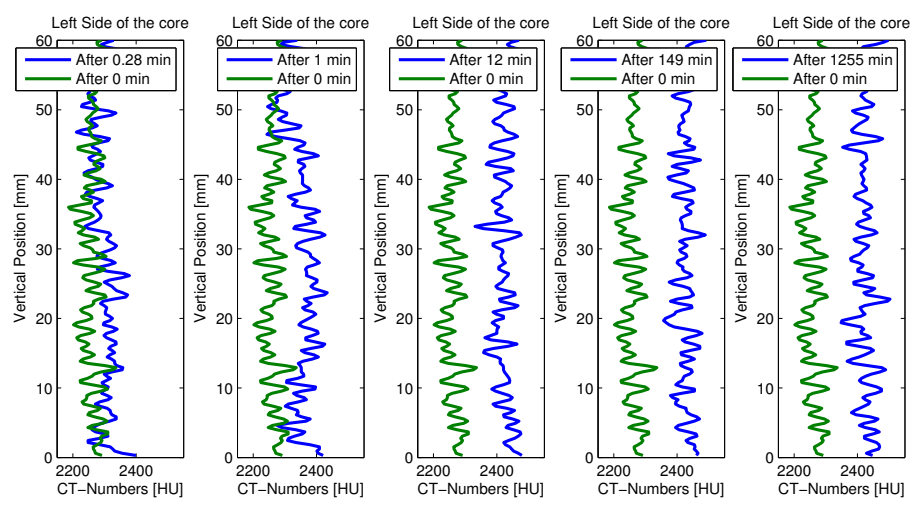

Figure 4.14: CT numbers at left side of the core in different time steps. Green represents initial condition, which has been shown for each plot. Blue represents the current condition.

Figure 4.14 shows at early time steps that the solvent penetrates from the bottom and the side almost equally. The rate of the solvent penetration from the top of the core is low compared to the penetration rate from the sides and bottom of the core.

\section{Middle section of the core}

Figure 4.15 illustrates the CT numbers at the middle section of the core for five different times. 

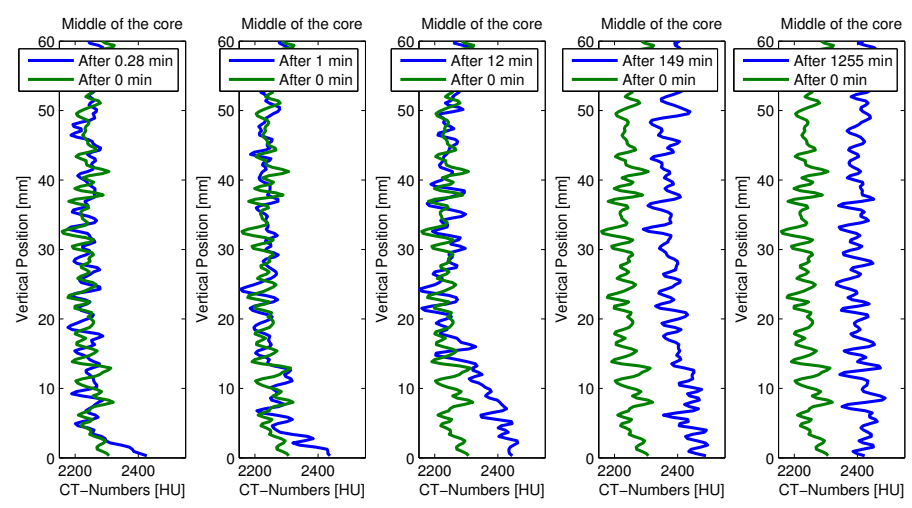

Figure 4.15: Variation of CT numbers at middle section of the core in different times. Green represents initial condition, which has been shown for each plot. Blue represents the current condition.

The increase of the CT numbers in the middle section are slower compared to the penetration from the sides of the core and increases from the bottom of the core. The CT numbers in these horizons show that the solvent penetrates into the core from the bottom of the core.

\section{Right side of the core}

Figure 4.16 illustrates the CT numbers at right side of the core for five different times. 

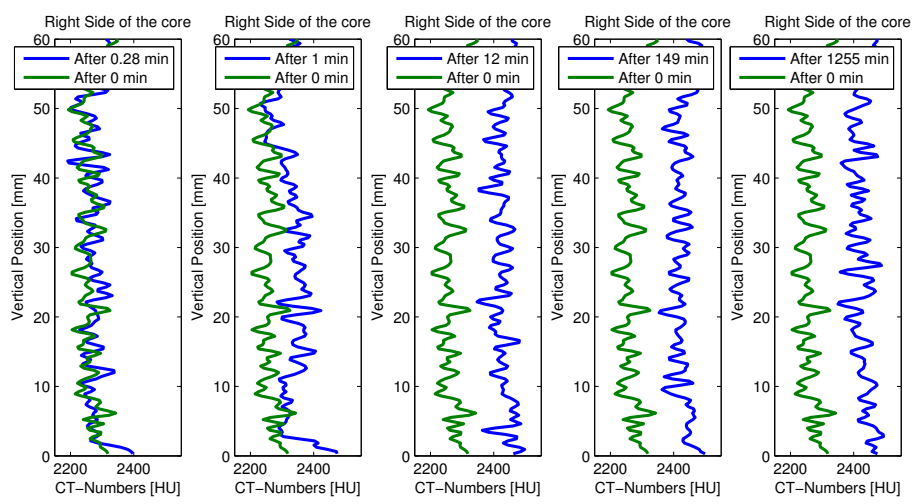

Figure 4.16: Variation of CT numbers at right of the core in different times. Green represents initial condition, which has been shown for each plot. Blue represents the current condition.

Note that the CT numbers at the right side of the core is same as at the left side.

Study of the of CT numbers in different vertical sections results in,

- The solvent penetrates into the core from sides equally at early time steps.

- The solvent gradually reaches to the middle section of the core. Figure 4.15 shows that the solvent penetrates into the core more from the bottom, because the CT numbers at this section increased from the bottom of the core as time increases.

- The faster changes of CT numbers at the right and left sides compared to the middle section shows that the penetration of the solvent from the sides is more significant.

\subsubsection{Averaging the CT-numbers along the core}

Averaging the CT numbers along the core gives us an idea about the evolution of the mass transfer between fracture and matrix. In this way we will have one average value for each row of pixels along the core. Because the distribution of pores and fluid along the core is not homogenous everywhere, we can subdivide the core into very small (e.g. $3 \mathrm{~mm}$ ) horizontal sections 
and determine the average of the CT-numbers in each part along the core instead of averaging for each row of pixels. In other words with this arithmetic averaging we would have one averaged value for each section and we can see how the concentration of solvent varies with time along the core.

For averaging the CT-numbers at each horizon the core part should be cropped from the entire image. Figure 4.17 shows an example of a cropped image.

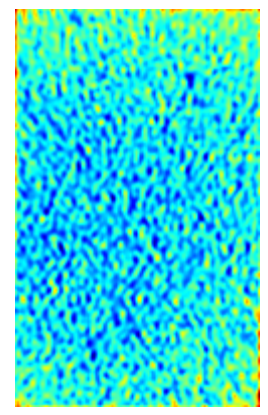

Figure 4.17: An example of cropped core image, taken in one of our experiments 
In the next step the core is subdivided into $3 \mathrm{~mm}$ sections. The average of $\mathrm{CT}$ numbers in each section is determined. In this way a better distribution of pores and grains is obtained. Therefore for each section along the core there would be an averaged CT-number. If these average values are plotted in vertical direction along the core, Figure 4.18 would be produced.

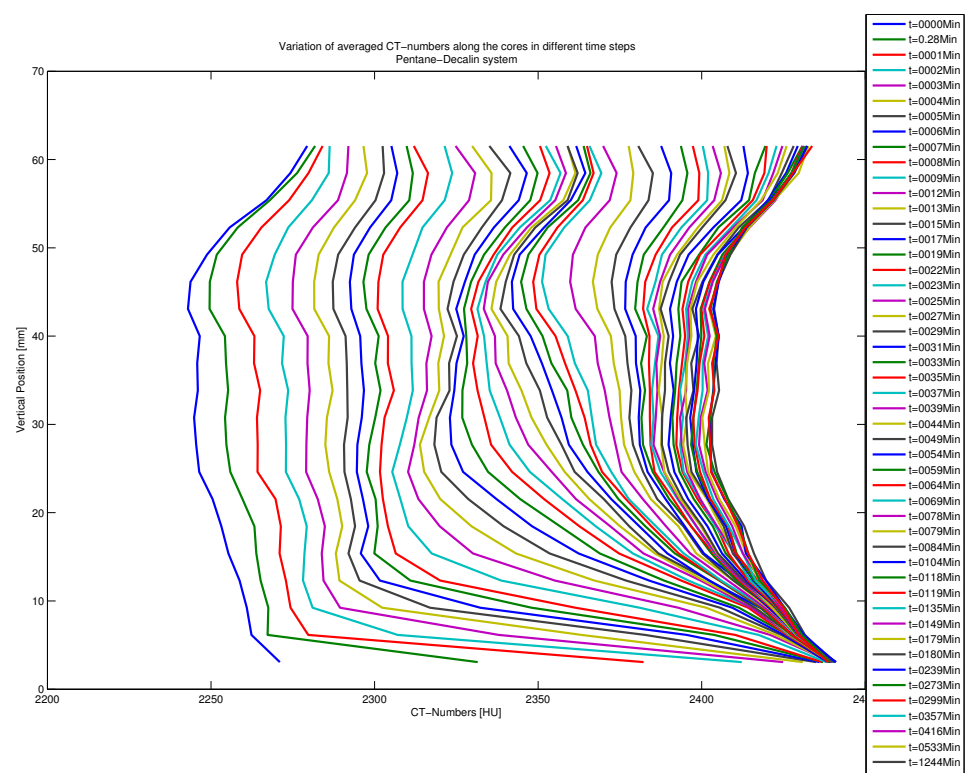

Figure 4.18: Variation of averaged CT numbers along the core in different time steps - Pentane-Decalin system 
Figure 4.18, shows the evolution of the process clearly. There is a significant change in CT-numbers with time, which means that the fluid properties inside the core has changed due to the mass transfer between the fracture and the matrix. It can be interpreted from Figure 4.18 that the solvent penetrates into the core more from the bottom, because the changes of CTnumber at the bottom of the core is faster and more significant. The reason for this behavior is the higher density of the solvent compared to the oil in the matrix, which makes the solvent penetrates into the core from bottom and produce the oil from top of the core. The time intervals increase with evolution of the process. Figure 4.18 clearly shows that most of the transfer process is at early times. After 10 minutes, when the variation of fluid at the bottom becomes slower, the solvent reaches the top of the core and starts to mix with the fluid inside the core.

\subsubsection{Saturation Calculation}

The last step of the data analysis is to calculate the saturation of solvent in the core and the oil recovery. For ease of reference we mention the corresponding equations in this section again. CT numbers can be converted to the saturation using Eq.4.1,

$$
\mathrm{S}_{\text {Solvent }}=\frac{\mathrm{CT}_{\text {exp }}-\mathrm{CT}_{\text {oil }}}{\mathrm{CT}_{\text {solvent }}-\mathrm{CT}_{\text {oil }}}
$$

where $\mathrm{CT}_{\text {oil }}$ is the CT number of the fully saturated core with the oil which can be obtained from the first scan and $\mathrm{CT}_{\text {solvent }}$ is the $\mathrm{CT}$ number of the fully saturated core with the solvent. For constructing the CT numbers of the fully saturated core with the solvent it is assumed that a small region in the bottom of the core at the end of the experiment is fully saturated by the solvent. Subsequently, the same region is taken from the first time step to determine the ratio between the $\mathrm{Ct}$ numbers of the solvent and the oil. The calculated ratio and the CT numbers of the core fully saturated with the oil are used to construct the CT numbers of the core fully saturated with the solvent.

Using Eq.4.1, we can calculate the average solvent saturation inside the core at different times. Figure 4.19 illustrates the solvent saturation and its evolution with time along the core for Pentane-Decalin system. 


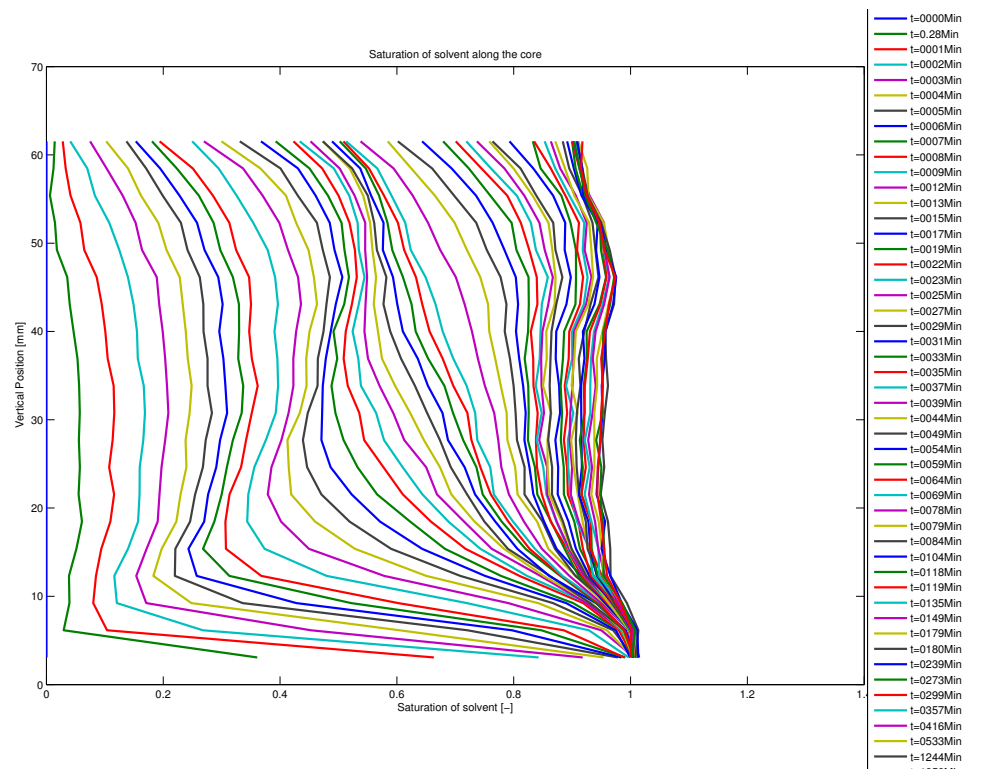

Figure 4.19: Variation of average of solvent saturation along the core in different time steps - Pentane-Decalin system

Moreover Figure 4.19 indicates that the saturation of the solvent increases from the bottom of the core. Because the increase of the CT numbers at the bottom is faster than the top.

\subsubsection{Recovery}

Assuming that conservation of fluid volume results in equality of oil produced from the core and the volume of the invaded solvent in the core Eq.4.2 can be used to determine the recovery,

$$
\text { \%Solvent }=\% \text { Produced Oil }=\frac{\mathrm{CT}_{\text {exp }}-\mathrm{CT}_{\text {oil }}}{\mathrm{CT}_{\text {solvent }}-\mathrm{CT}_{\text {oil }}}
$$

By averaging the produced oil of different sections of the core in different time steps we can construct the recovery profile. Figure 4.20 illustrates the recovery of Pentane from the matrix. 


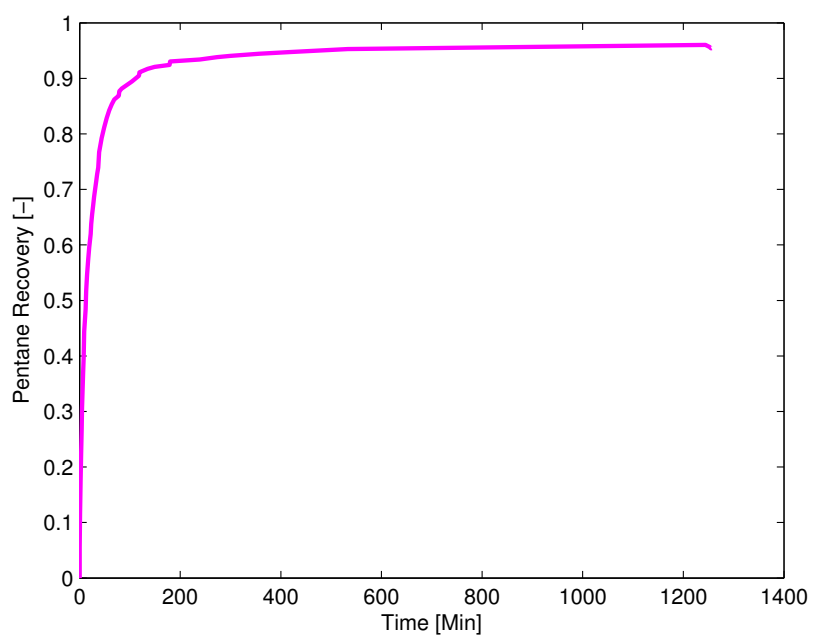

Figure 4.20: Recovery profile of Pentane

The recovery profile is obtained using the saturation of the solvent ( produced oil) in the core assuming the equality of produced oil and solvent saturation. To cross check the validity of the recovery calculation we calculate the saturation of the oil in the bulk at the end of the experiment using a calibration profile. For constructing the calibration curve different compositions of Decalin and Pentane were made and scanned with the CT scanner. Figure 4.21 shows the CT numbers of different mixtures of Decalin and Pentane, which follows a straight line. To determine the composition of the mixture at the end of the experiment, the CT number of bulk in the last time step was determined. Subsequently composition of the mixture of the bulk was read from the curve of Figure 4.21 based on the CT number. 


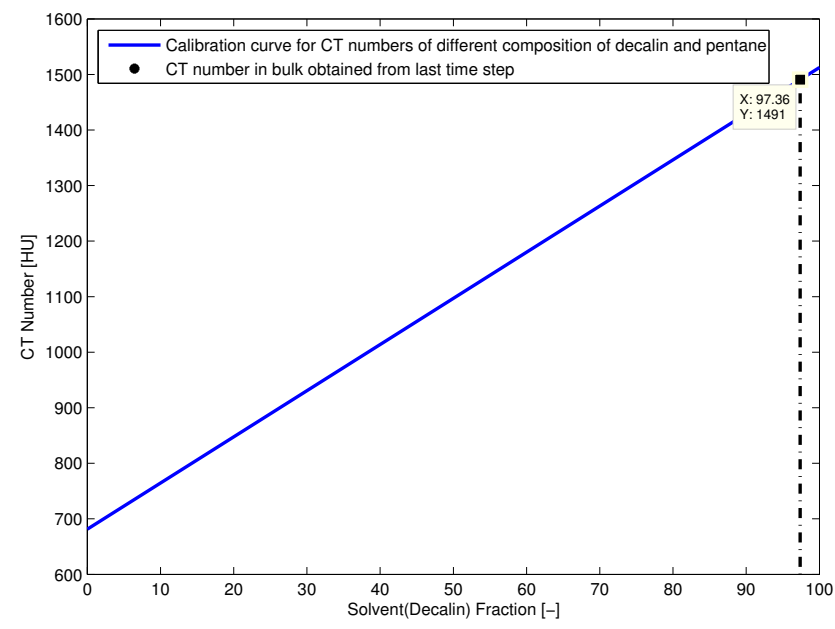

Figure 4.21: CT number Vs. different composition of Decalin and Pentane

At this step it is possible to compare the last point saturation obtained from the bulk and one obtained from the saturation of solvent inside the core. Figure 4.22 illustrates the recovery profile and the last point saturation obtained from the bulk, which is in a good agreement with the recovery profile obtained from the CT-numbers inside the core.

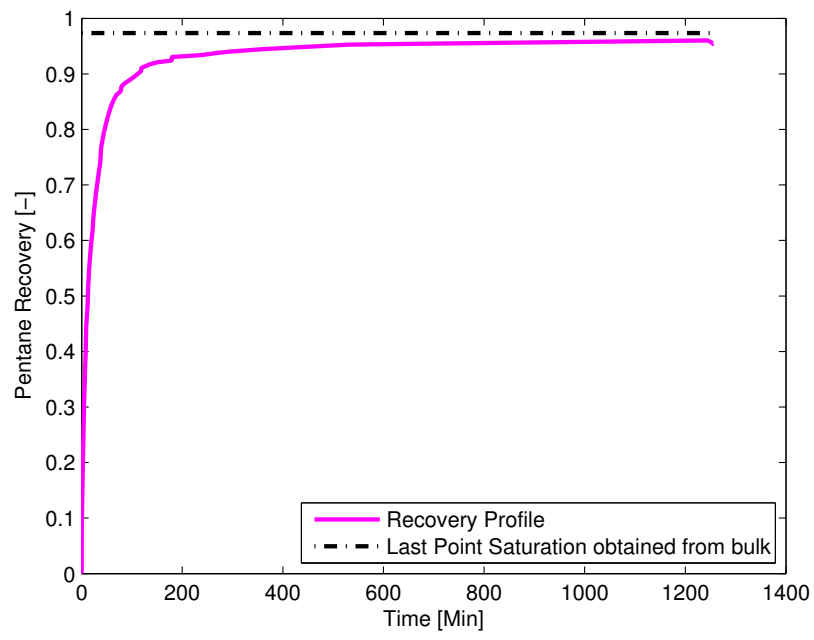

Figure 4.22: Pentane Recovery and last point recovery obtained from the bulk 


\subsubsection{Visualization of the process}

One of the objectives of this thesis is the visualization of the interaction between the fracture and the matrix. Following figures illustrate the CT images at different times. Note that the blue color is the oil inside the core and yellow color is the solvent.

As shown in Figure 4.23 the solvent starts invading the core from the bottom and the sides of the core and gradually travels to the centre of the core. Because of the low viscosity of the oil and high permeability of the core the recovery process is fast. 

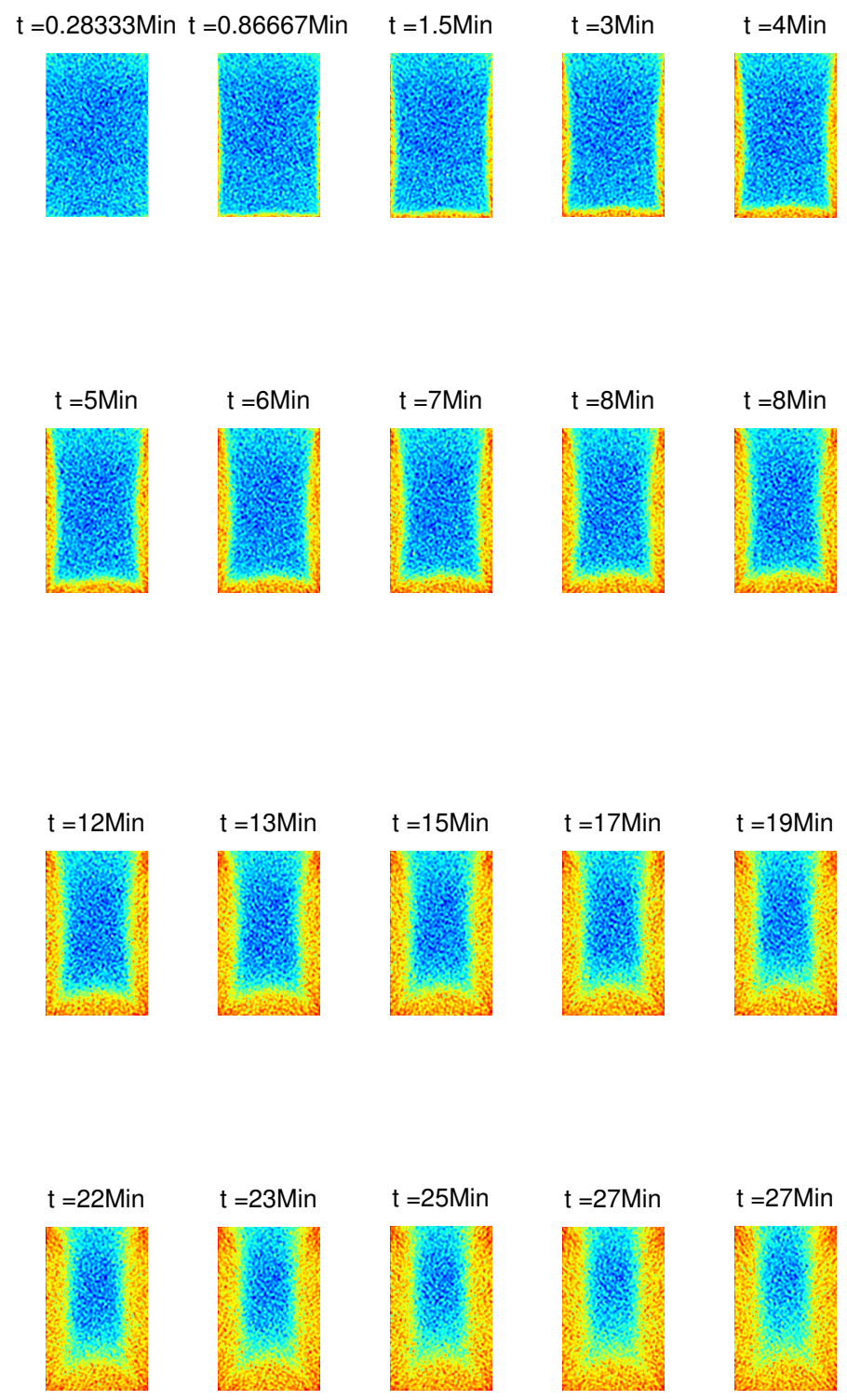

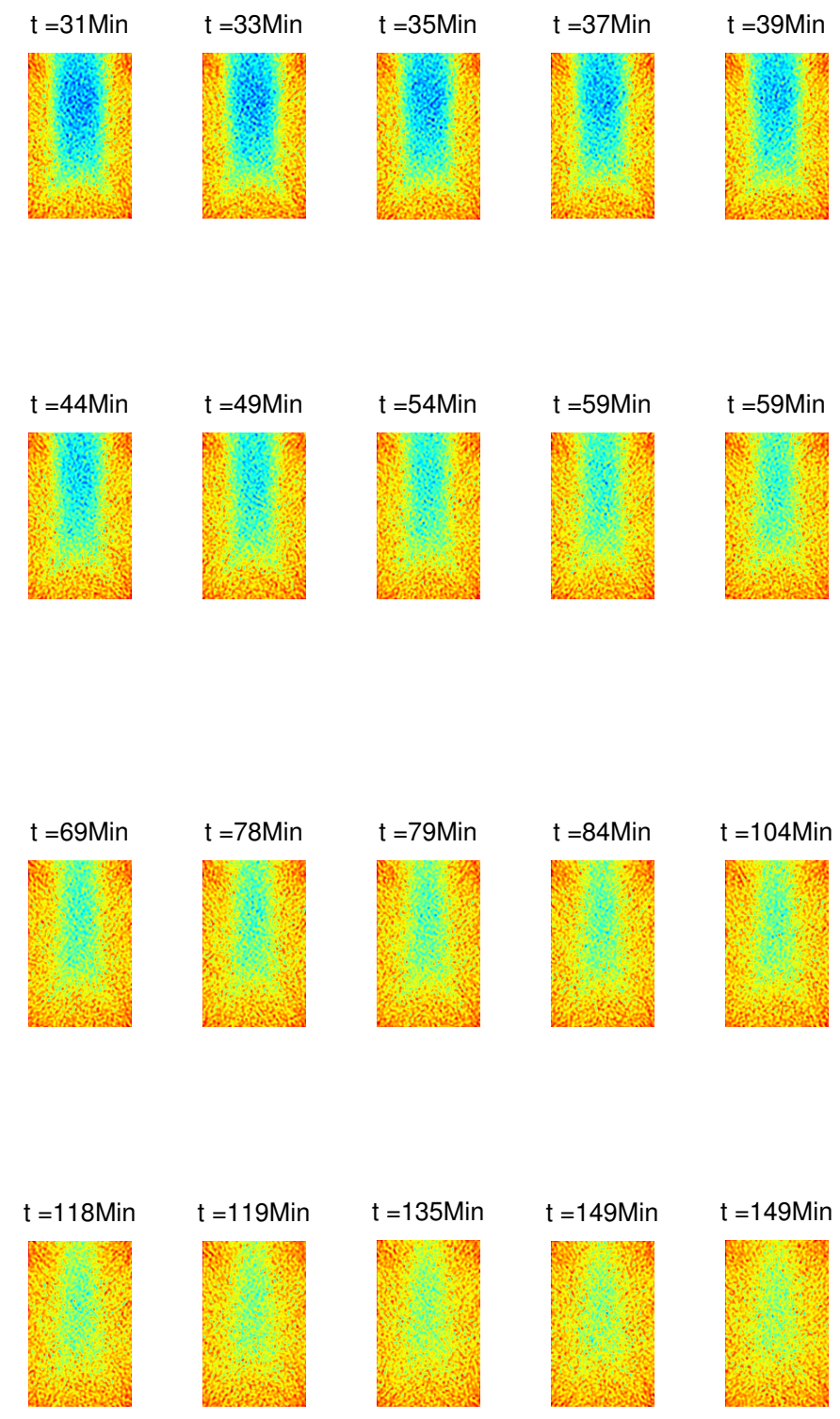

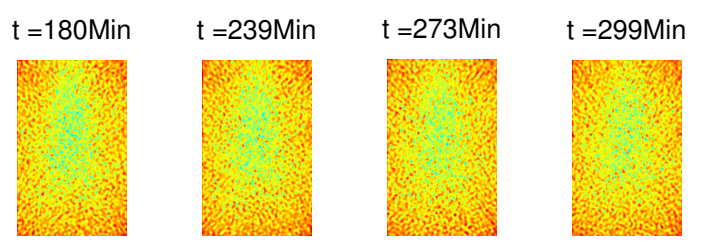

$\mathrm{t}=357 \mathrm{Min}$
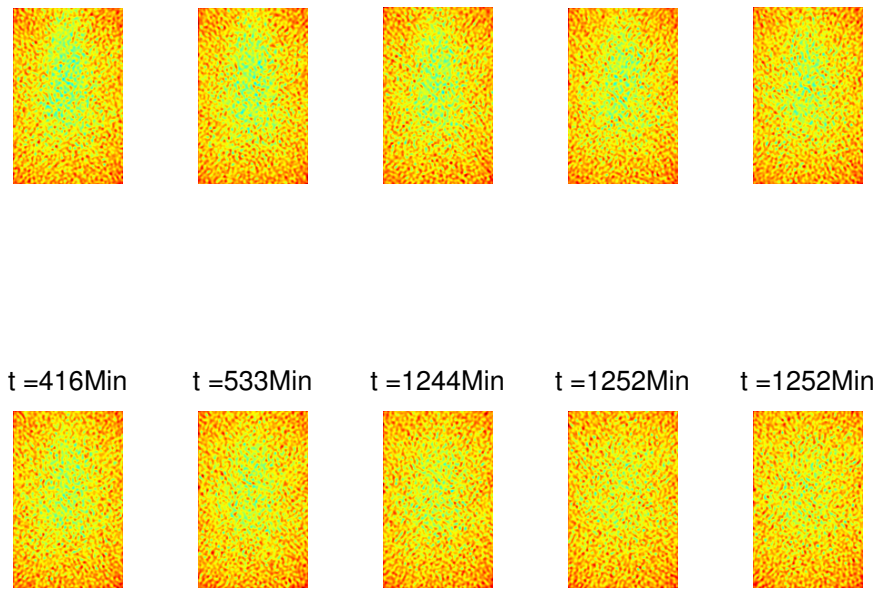

Figure 4.23: CT-images of the penetration of Decalin from the fracture into the core saturated with Pentane. Note that he blue color is Pentane inside the core and yellow color is Decalin. 


\subsection{Hexadecane-Decalin}

In the second study, we conducted an experiment with Decalin in the environment around the core plug as solvent and the core was saturated with hexadecane as model oil. The fluid properties are provided in Chapter 3 of this report. Hexadecane is more viscous, $3.507 \mathrm{cP}$, than the Pentane, $0.24 \mathrm{cP}$. The viscosity of Hexadecane is also higher than the viscosity of the Decalin. The density of hexadecane is higher than Pentane but lower than Decalin. Therefore it is expected that the solvent (denser fluid) penetrates into the core again from bottom. Following the results of this experiment is provided. The procedure of data analysis is same as what we did for Pentane-Decalin system.

\subsubsection{CT numbers at different horizons along the core}

Same as the previous system, first of all we study how the CT numbers change in different positions of the core to investigate how the solvent penetrates from the fracture into the core. Figure 4.24 and Figure 4.25 illustrate the CT numbers in three horizontal and vertical sections respectively after 62 minutes. More explanation about data processing is provided in the Pentane-Decalin section.

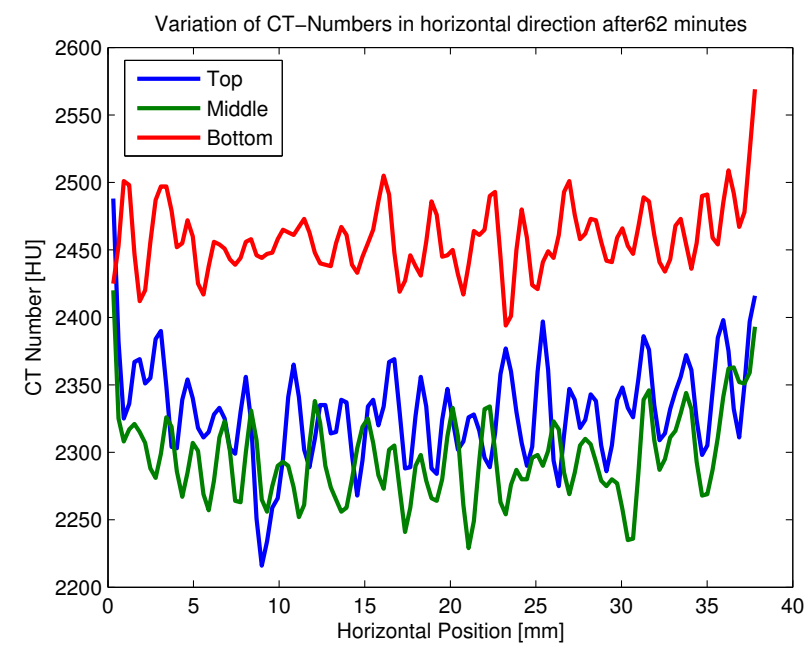

Figure 4.24: CT-numbers in three different horizontal position 62 minutes after the start of the experiment 
In the same was as the Pentane-Decalin system, the CT-numbers at the bottom of the core is significantly higher than at the top and middle of the core. The CT-numbers at the top of the core is slightly higher than at the middle of the core. Higher CT number at the bottom of the core shows that the density of the fluid is higher at the bottom than at the top, which means that the solvent invades more from the bottom.

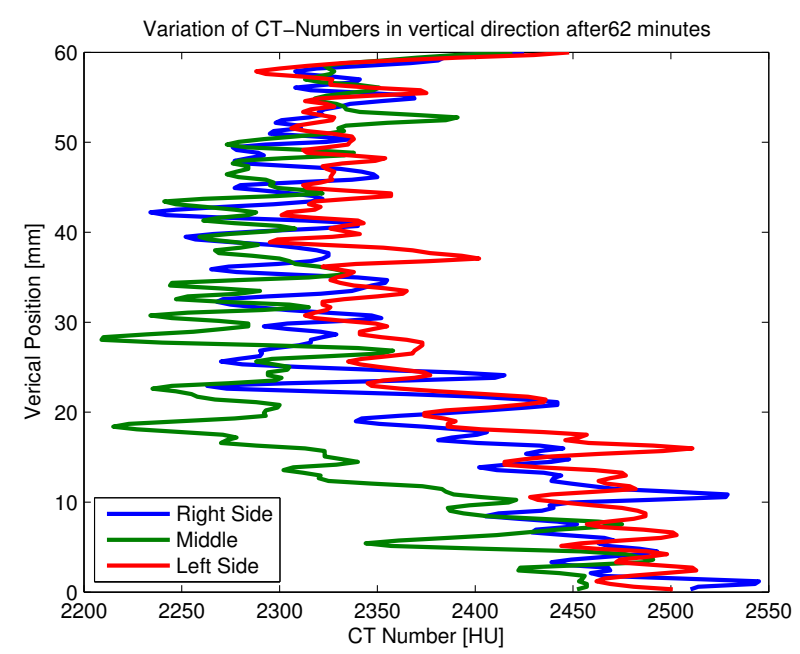

Figure 4.25: CT-numbers in three different vertical position 62 minutes after the start of the experiment

Figure 4.25 clearly shows that the invasion of solvent from the fracture into the core happens more from the bottom and the sides, because at the bottom of the core the CT numbers at the right and the left side of the core are higher than at the middle part of the core. But note that the penetration of solvent from the sides is not equal along the core and more pronounced at the bottom part than at the top part, whereas in the Pentane-Decalin case the solvent penetrates from the sides of the core more equally. This behavior is clearly shown in the Section 4.3.2. 


\subsubsection{Comparison of CT numbers in horizontal and vertical sections in different time steps}

Evolution of the process can be studied by comparing the CT numbers along the core in different time steps. For this reason the CT numbers in different sections are plotted in 5 different times. The position of these sections are shown schematically in Figure 4.6 and Figure 4.8.

\section{Top of the core}

Figure 4.26 illustrates the difference between CT-numbers at top of the core at five different time steps due to mass transfer between solvent in the fracture and the oil in the core. The variation of CT numbers in different positions for all time steps are provided in the appendix.

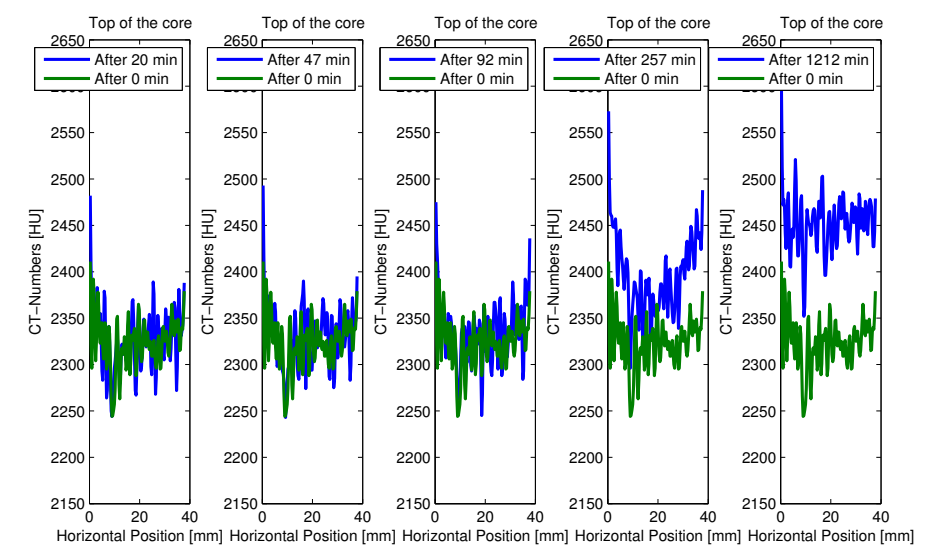

Figure 4.26: CT numbers at top of the core in different times

As shown in the Figure 4.26 the CT numbers at the top of the core do not change before 92 minutes. However, gradually after 257 minutes, it starts increasing from sides of the core.

\section{Middle of the core}

Figure 4.27 illustrates the variation of the CT numbers at the middle of the core for five different times. 


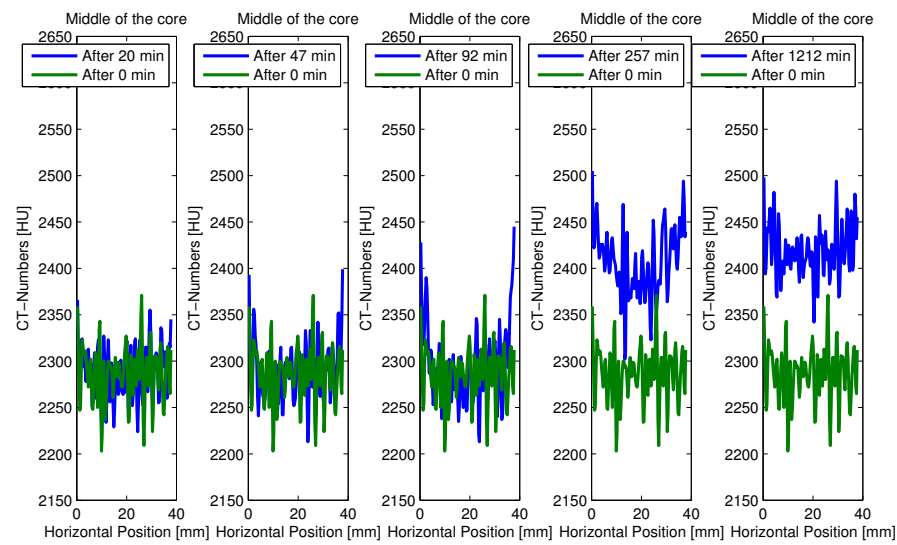

Figure 4.27: CT numbers at middle of the core in different times

In the middle section of the core, the solvent penetrates into the core from the sides and slowly reaches to the center of the core.

\section{Bottom of the core}

Figure 4.28 illustrates the CT numbers at bottom of the core for five different times.

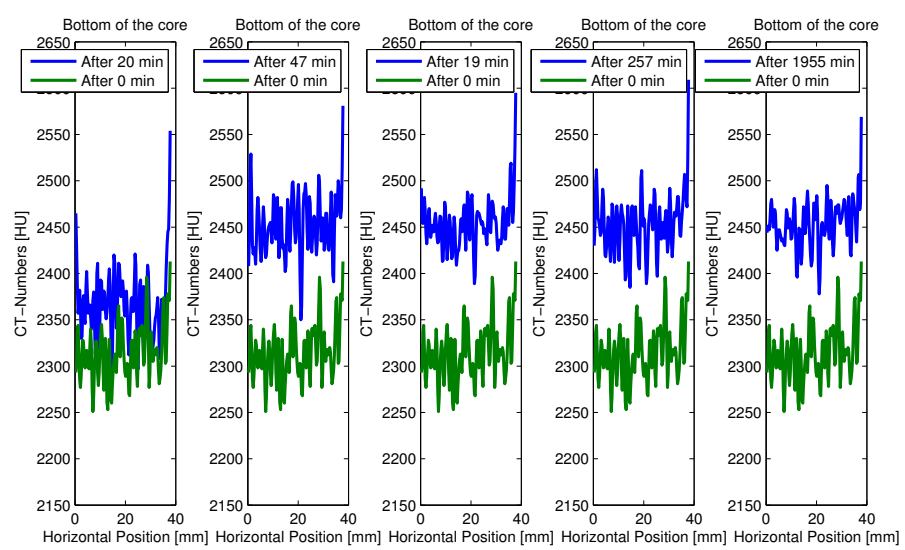

Figure 4.28: CT numbers at bottom of the core in different times

Figure 4.28 shows significant changes in CT numbers from early times. The following matters can be interpreted from comparing CT numbers in different 
positions:

- The increase of the CT numbers at the bottom of the core are much faster than at the top and middle part of the core. The invasion of the solvent takes place from the bottom part of the core.

- In the middle and top of the core the CT numbers change more from the sides. In other words, in these positions the solvent penetrates into the core more from the sides and gradually reaches the center of the core.

- Increase of the CT numbers at the middle part of the core are more than at the top of the core.This shows that gravitational forces are more dominant than the diffusion forces.

- The penetration of solvent from the sides is not as much as the PentaneDecalin case. In the Pentane-Decalin case, the solvent start penetrating into the core from the sides and the bottom of the core, whereas in the hexadecane-Decalin system the process starts with the solvent penetration from the bottom part of the core. This also confirms the dominance of the gravitational forces during displacements.

In order to investigate how the solvent penetrates into the core from sides of the core we compare the CT numbers in vertical sections in different time steps.

\section{Left side of the core}

Figure 4.29 illustrates the CT numbers at the left side of the core for five different times. 


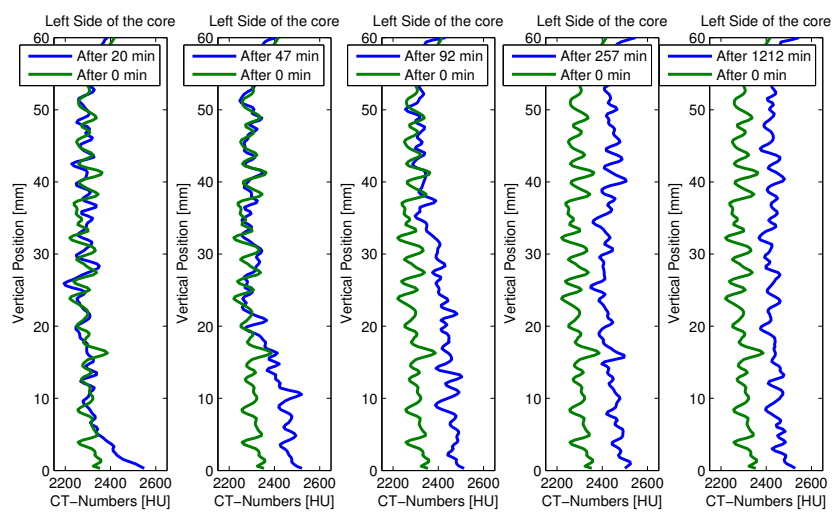

Figure 4.29: CT numbers at left side of the core in different times

In contrast with the Pentane-Decalin system, the penetration of solvent in the hexadecane-Decalin system is not equal from the sides of the core. It starts penetrating into the core from the bottom side of the core and gradually moves to the top part of the core.

\section{Middle section of the core}

Figure 4.27 illustrates the CT numbers at the middle section of the core for five different times.

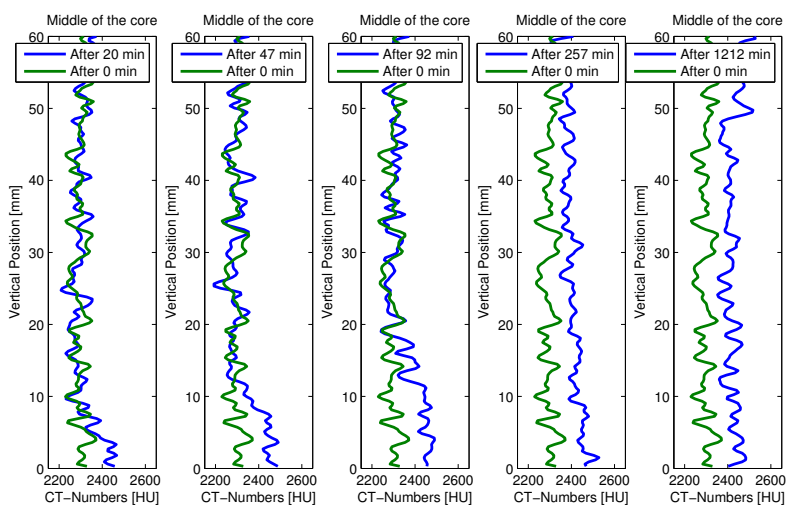

Figure 4.30: CT numbers at middle section of the core in different time steps 
In the middle section of the core, the solvent penetrates gradually from the bottom part.

\section{Right side of the core}

Figure 4.31 illustrates the CT numbers at the right side of the core for five different times.

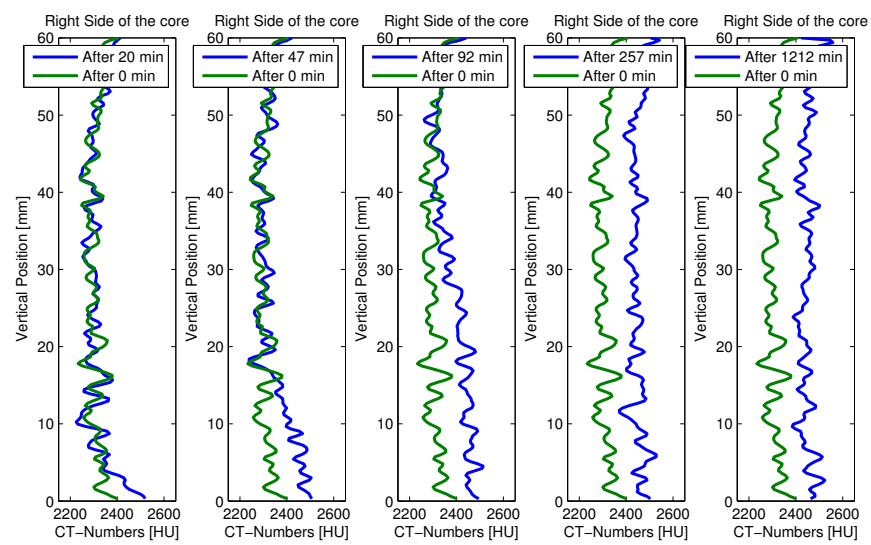

Figure 4.31: $\mathrm{CT}$ numbers at right side of the core in different times

The CT number in the right side of the core is same as the left side.

Study of the variation of CT numbers in different vertical sections results in,

- In contrast with the Pentane-Decalin system, in the hexadecane-Decalin case the solvent did not penetrate equally from the sides. The solvent started penetrating into the core from the bottom of the core after 47 minutes and gradually moved to the top part of the core.

- In the middle section of the core, the penetration of the solvent took place later than the sides. In this section the solvent penetrated into the core from the bottom of the core as well.

- After 257 minutes the CT numbers at the sides of the core do not change, which means the sides of the core are completely invaded by the solvent. 


\subsubsection{Averaging the CT-numbers along the core}

As explained in the Pentane-Decalin section, the next step in the experimental analysis is dividing the core into the very small sections and averaging the CT numbers in each section. Then we can study the evolution of the process by using these results. Figure 4.32 illustrates the variation of the averaged CT numbers along the core at different times.

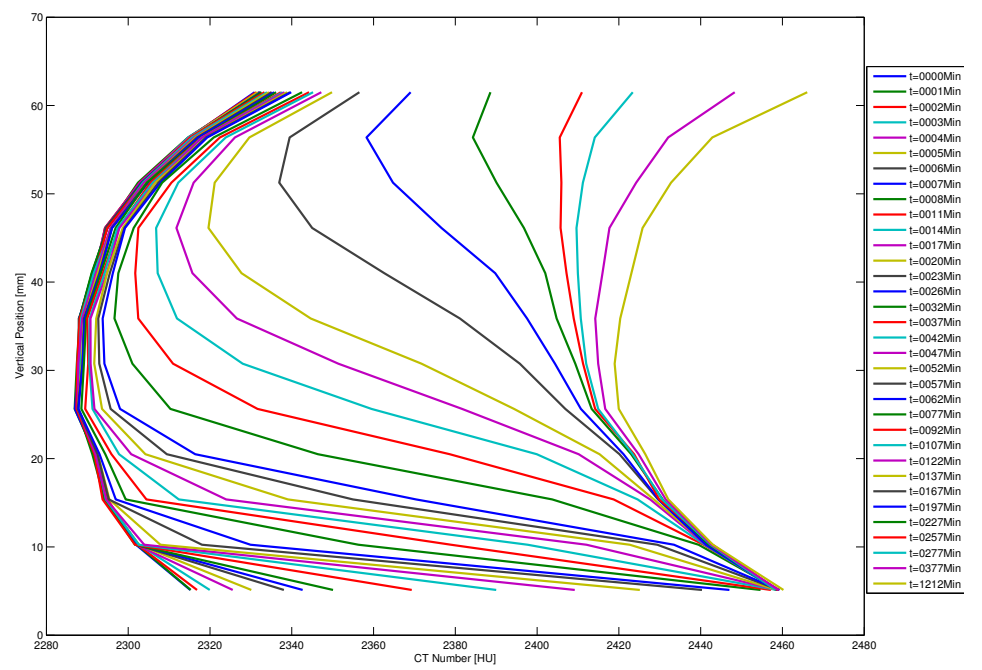

Figure 4.32: Averaged CT numbers along the core in different times Hexadecane-Decalin system

The penetration of the solvent can be clearly observed at Figure 4.32. The changes of CT numbers at different times show the changes of the fluid properties inside the core. In other words, the mass transfer between fracture and the matrix results in the variation of the $\mathrm{CT}$ numbers inside the core. Figure 4.32 indicates that in the early time steps the process is slow and after 20 minutes the solvent starts penetrating into the core from the bottom. Significant and fast changes of CT numbers at the bottom of the core shows this matter. The reason for this behavior is the higher density of the solvent compared to the oil in the matrix, which makes the solvent penetrates into the core more from the bottom and produce the oil from the top of the core, i.e., displacement is governed by gravity. 


\subsubsection{Saturation Calculation}

At this stage we calculate the saturation of the solvent inside the core using Eq.4.1. Figure 4.33 illustrates the solvent saturation inside the core in different times.

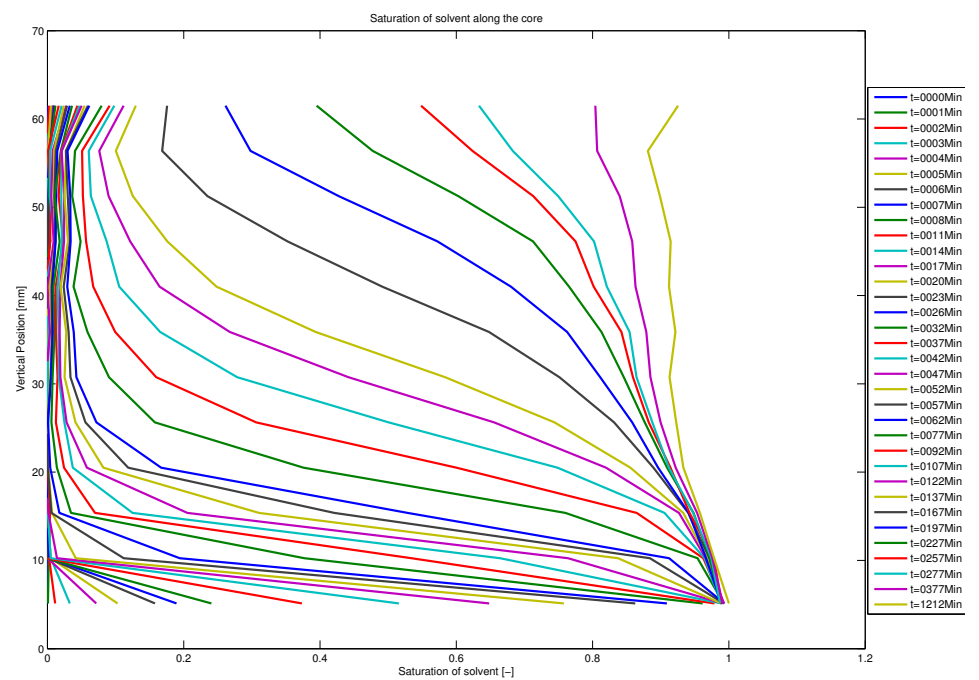

Figure 4.33: Average of solvent saturation along the core in different times Hexadecane-Decalin system

Figure 4.19 shows that the saturation of the solvent does not change significantly at early time steps. As can be seen the solvent saturation increases gradually from the bottom of the core and vertical distribution of the solvent is not as uniform as in the Pentane-Decalin case. Non-constant vertical distribution of solvent along the core suggests that the displacement is governed by gravity.

\subsubsection{Recovery}

The Recovery of Hexadecane could be calculated by assuming the conservation of fluid, using eq.4.2. Recovery profile is produced by averaging the produced oil ( saturation of solvent) at different time step. Figure 4.34 illustrates the recovery of Pentane from the matrix. 


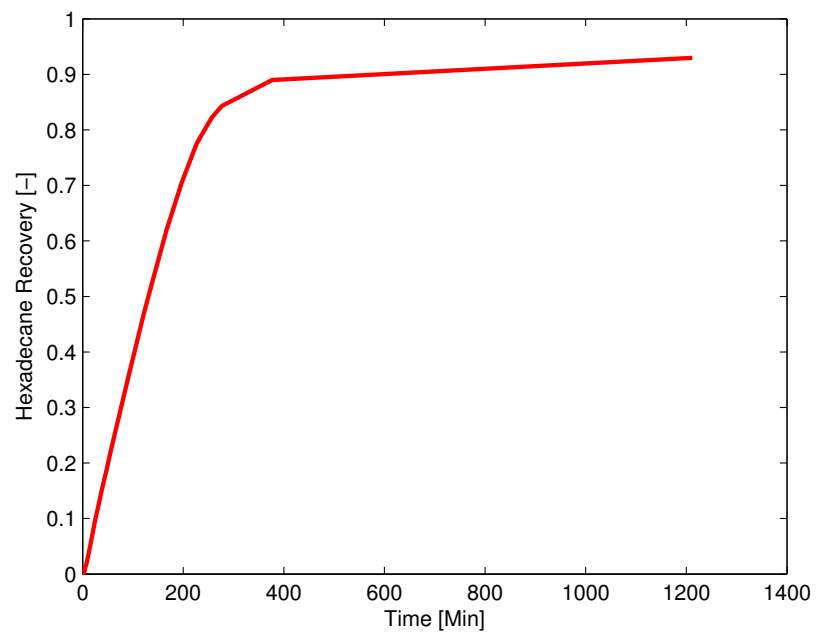

Figure 4.34: Recovery profile of hexadecane

As in the Pentane-Decalin case we can check the last point recovery using the CT data in the bulk at last time step. By last point recovery we mean that at the end of the process, the jar is opened and the fluid inside the jar was stirred to obtain a homogenous mixture and then the composition of this mixture can be determined using a calibration curve and the CT-number of the mixture. For constructing the calibration curve different compositions of hexadecane and Decalin was scanned. Figure 4.35 illustrates the CT number of different compositions of Decalin and hexadecane. Using the curve of figure 4.35 and the CT number of bulk in the last time step, we can determine the amount of produced oil at the last time step. 


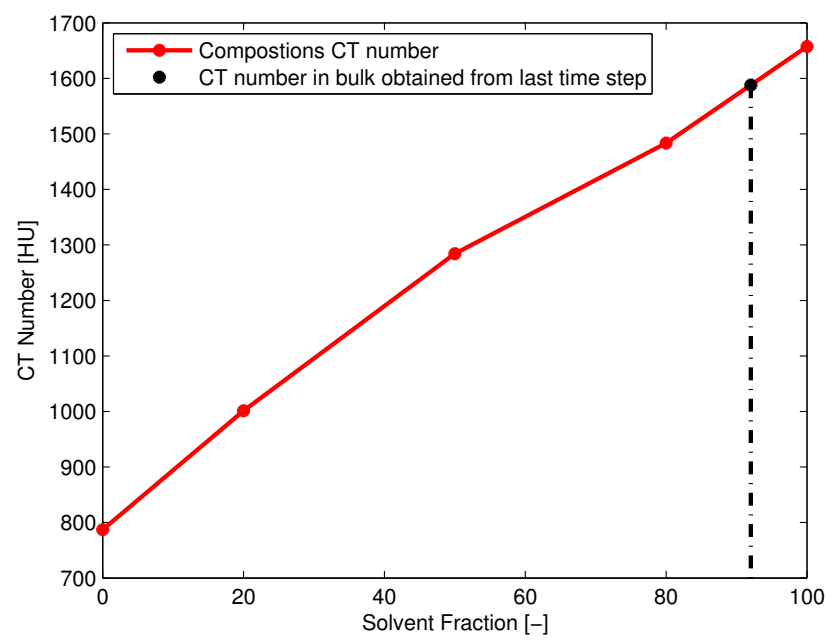

Figure 4.35: CT number Vs. different composition of Decalin and hexadecane

At this step it is possible to compare the last point saturation obtained from the bulk and one obtained from the saturation of solvent inside the core. Figure 4.36 illustrates the recovery profile and the last point recovery obtained from the bulk. 


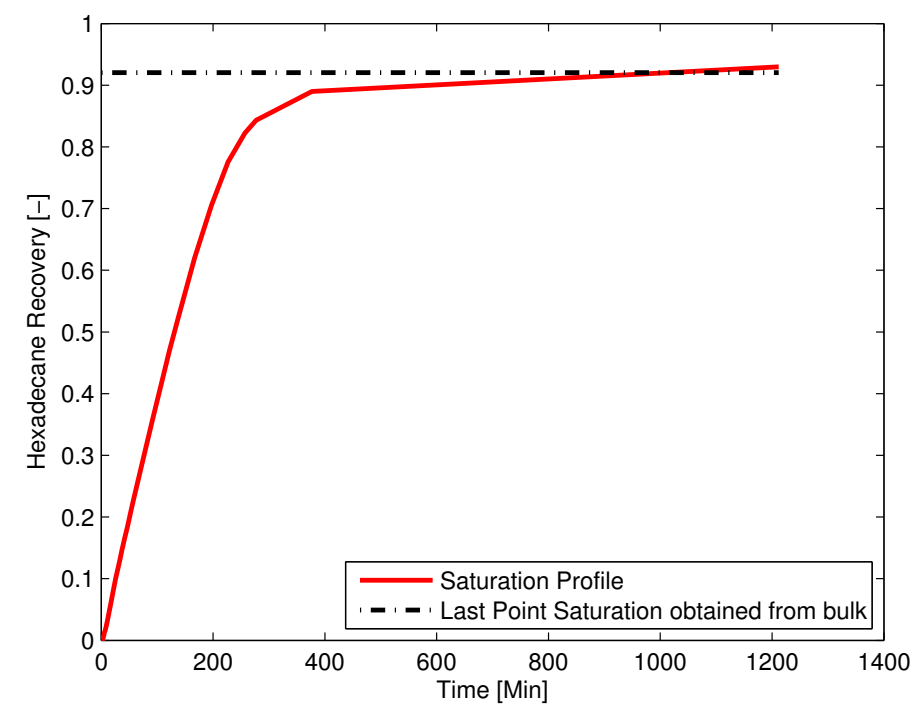

Figure 4.36: Hexadecane Recovery and last point recovery obtained from the bulk. By last point recovery we mean that at the end of the process, the jar is opened and the fluid inside the jar was stirred to obtain a homogenous mixture and then the composition of this mixture can be determined using a calibration curve and the CT-number of the mixture.

As interpreted from Figure 4.22 the last point saturation obtained from the bulk matches with last point of recovery profile. In this way we could validate the saturation calculation.

\subsubsection{Visualization of the process}

At this stage we visualize the whole process. Following figures illustrates the difference of CT images at different time steps which shows the state of process at that time steps. Note the green color is the oil inside the core and yellow color is the solvent.

As it is observable in the figure 4.37 solvent starts invading the core from bottom of the core. The solvent moves gradually from bottom of the core to top part of the core. In contrast with Pentane-Decalin case the penetration of the solvent from the sides is not that much and it more invades from bottom. 

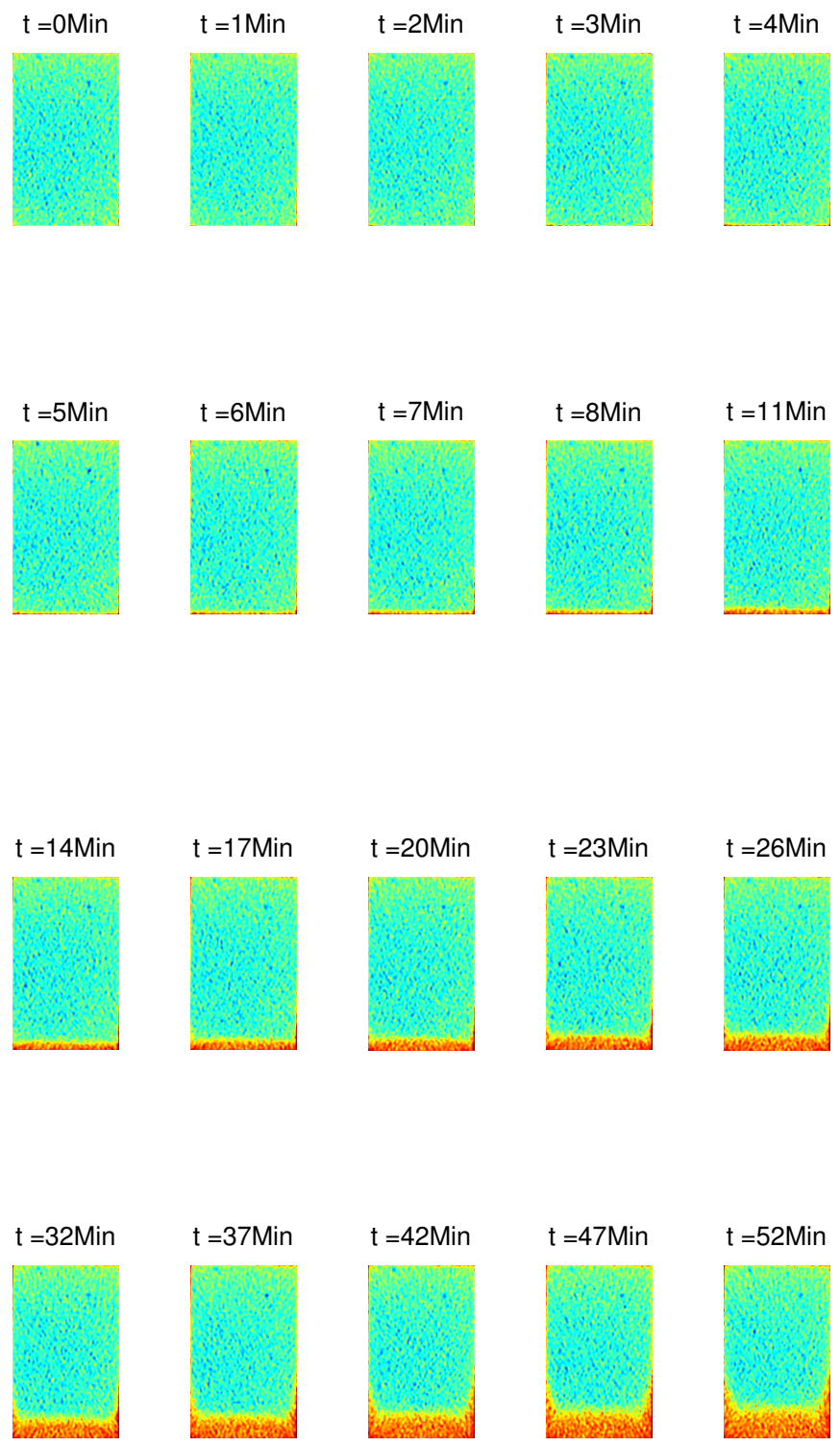

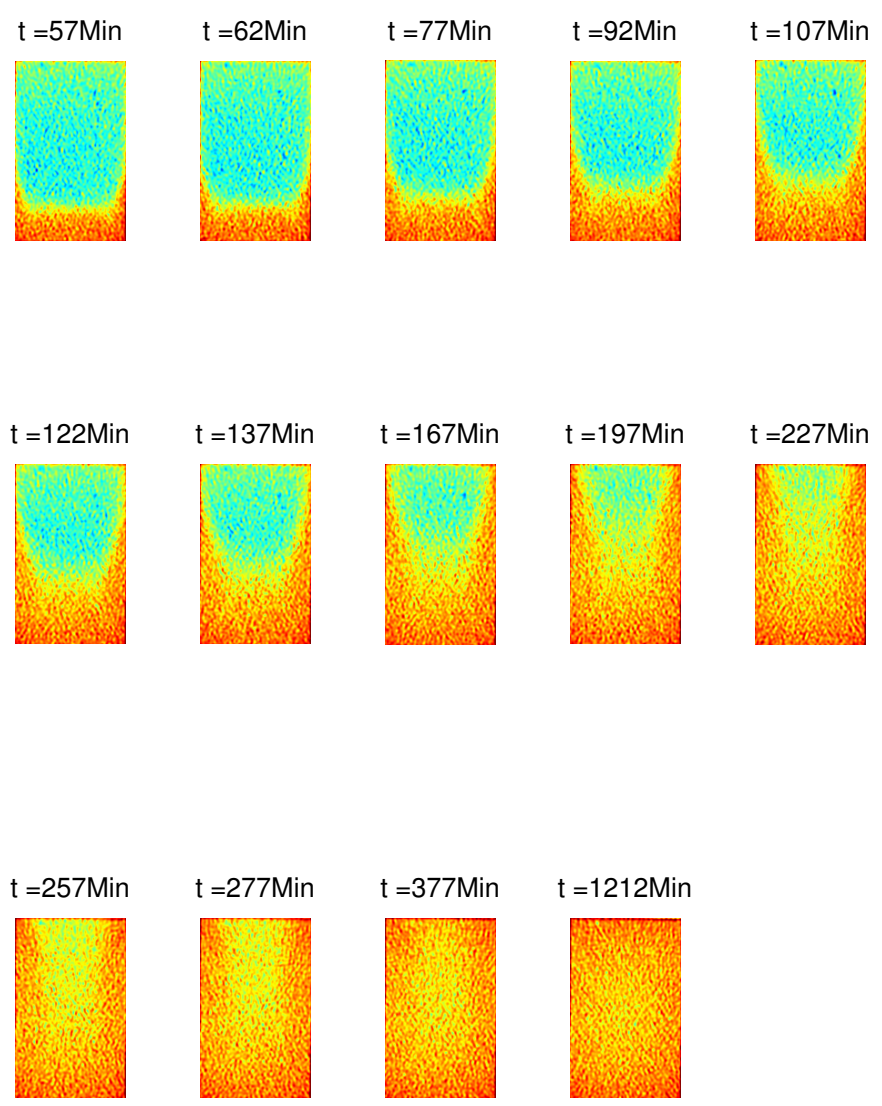

Figure 4.37: Visualization of penetration of Decalin from the fracture into the saturated core with hexadecane 


\subsection{Eicosane-Decalin}

We conducted an experiment using Eicosane, $\mathrm{C}_{20} \mathrm{H}_{42}$ as model oil and Decalin, $\mathrm{C}_{10} \mathrm{H}_{18}$ as solvent. Eicosane is solid at room temperature and its melting point is $36.7^{\circ} \mathrm{C}$. Therefore, for saturating the core with Eicosane we have to place the whole set up in a thermostatic bath in order to increase the temperature. After saturating the core, Eicosane inside the core became solid at room temperature. Afterward, the core was placed inside the jar containing doped solvent. The soak experiment was run for three days. The results of this experiment is provided below.

\subsubsection{Averaging the CT-numbers along the core}

As for the previous analysis the core is subdivided into small sections and the average of the CT numbers is determined in each section. Figure 4.38 shows the variation of $\mathrm{CT}$ numbers along the core with time.

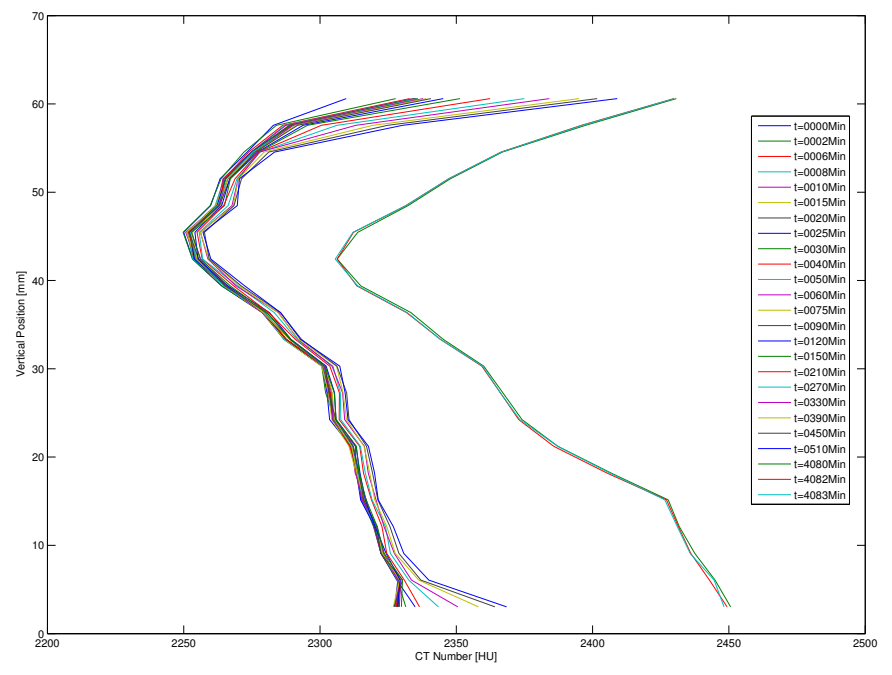

Figure 4.38: Variation of averaged CT numbers along the core in different time steps - Eicosane-Decalin system 
Figure 4.38 shows that the CT numbers at the top of the core do not follow a same trend as those at the bottom of the core from first time step. This fact shows that the distribution of the fluid at the top of the core is not same as at the bottom. This behavior could be due to two main reasons: a. Heterogeneity of the core sample and $\mathbf{b}$. imperfect saturation of the core. The most likely reason is the second one, because the core sample was selected from a homogenous Bentheimer sandstone block. Moreover, the previous experiments did not show any significant heterogeneity along the core. The imperfect saturation could have different reasons: a. The amount of available Eicosane was not sufficient for $100 \%$ saturation of the core. $\mathbf{b}$. The volume of Eicosane changed due to phase changes. c. The pressure used for the saturation was not high enough to displace air inside the core.

Despite of imperfect saturation of the core, studying the CT numbers along the core can provide us with some useful information. Figure 4.38 shows that the CT numbers do not change significantly after 510 minutes, i.e., the process is very slow compared to the previous experiments. The CT numbers in first 510 minutes are almost the same for each time step, which means that the solvent penetrates into the core equally from each side of the core. In last time steps the CT numbers are higher at the bottom of the core. It can be concluded that gravity does not play an important role at the beginning of the experiments, however at the end of the experiments it helps the The saturation profile can give us a better understanding of the solvent penetration.

\subsubsection{Saturation Calculation}

At this stage we calculate the saturation of the solvent inside the core using eq.4.1. Figure 4.39 illustrates the solvent saturation inside the core at different times. 


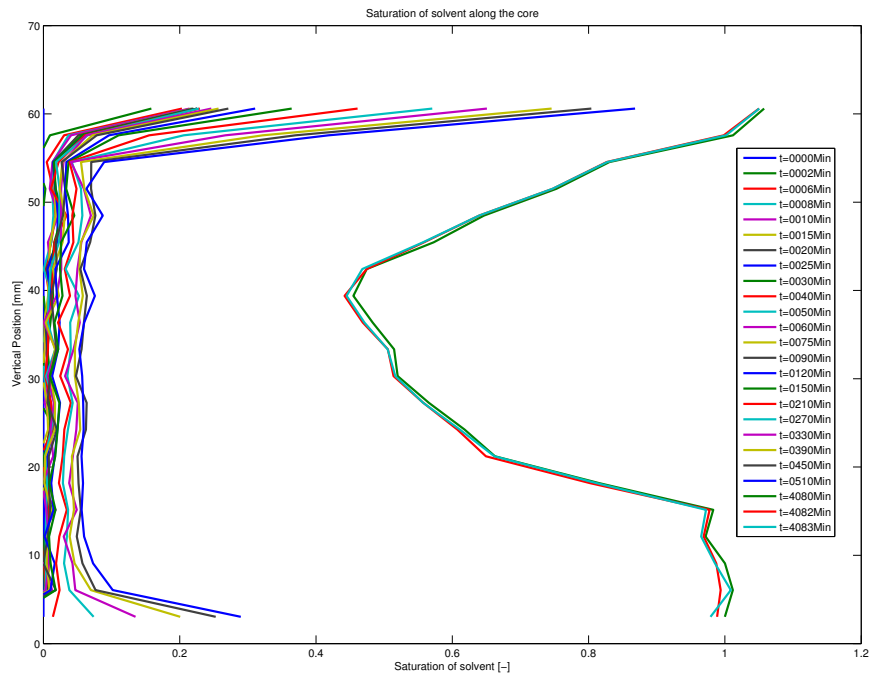

Figure 4.39: Variation of average of solvent saturation along the core in different time steps - Eicosane-Decalin system

The experiment shows a uniform vertical solvent distribution. Vertical symmetry of the saturation profile suggests that the displacement is governed by pure diffusion and not by gravity in the first time steps; however after 2 days gravity enhanced the penetration of the solvent.

\subsubsection{Recovery}

The recovery of Hexadecane could be calculated by assuming the conservation of fluid, using eq.4.2. The Recovery profile is produced by averaging the produced oil ( saturation of solvent) at different times. Figure 4.40 illustrates the recovery of Eicosane from the matrix. 


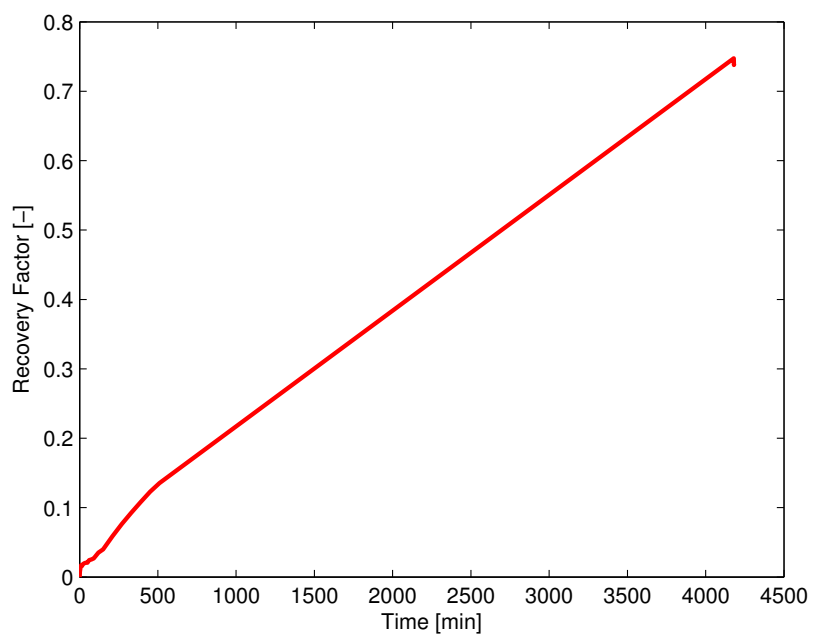

Figure 4.40: Recovery profile of Eicosane

The recovery profile shown in Figure 4.40 indicates that the underlying process for producing Eicosane from the core is very slow, even after 3 days Eicosane is not recovered completely.

\subsubsection{Visualization of the process}

At this stage we visualize the whole process. Following figures illustrate the difference of CT images at different times. The state of the process at each time can be interpreted from these figures. Note that the green color represents Eicosane inside the core and yellow color is Decalin.

Blue region at the top of the core in Figure 4.41 shows that the core is not saturated by Eicosane perfectly. This blue region could be air that is trapped inside the core. Low CT numbers at the top of the Figure 4.38 is due to presence of this region. As it is observable in the Figure 4.41 before 120 minutes almost nothing happened and gradually after that the solvent started to penetrate into the core equally from each side of the core. After 4080 minutes the concentration of the solvent is higher at the bottom of the core, which shows that at later time steps gravity enhanced the penetration of the solvent. 

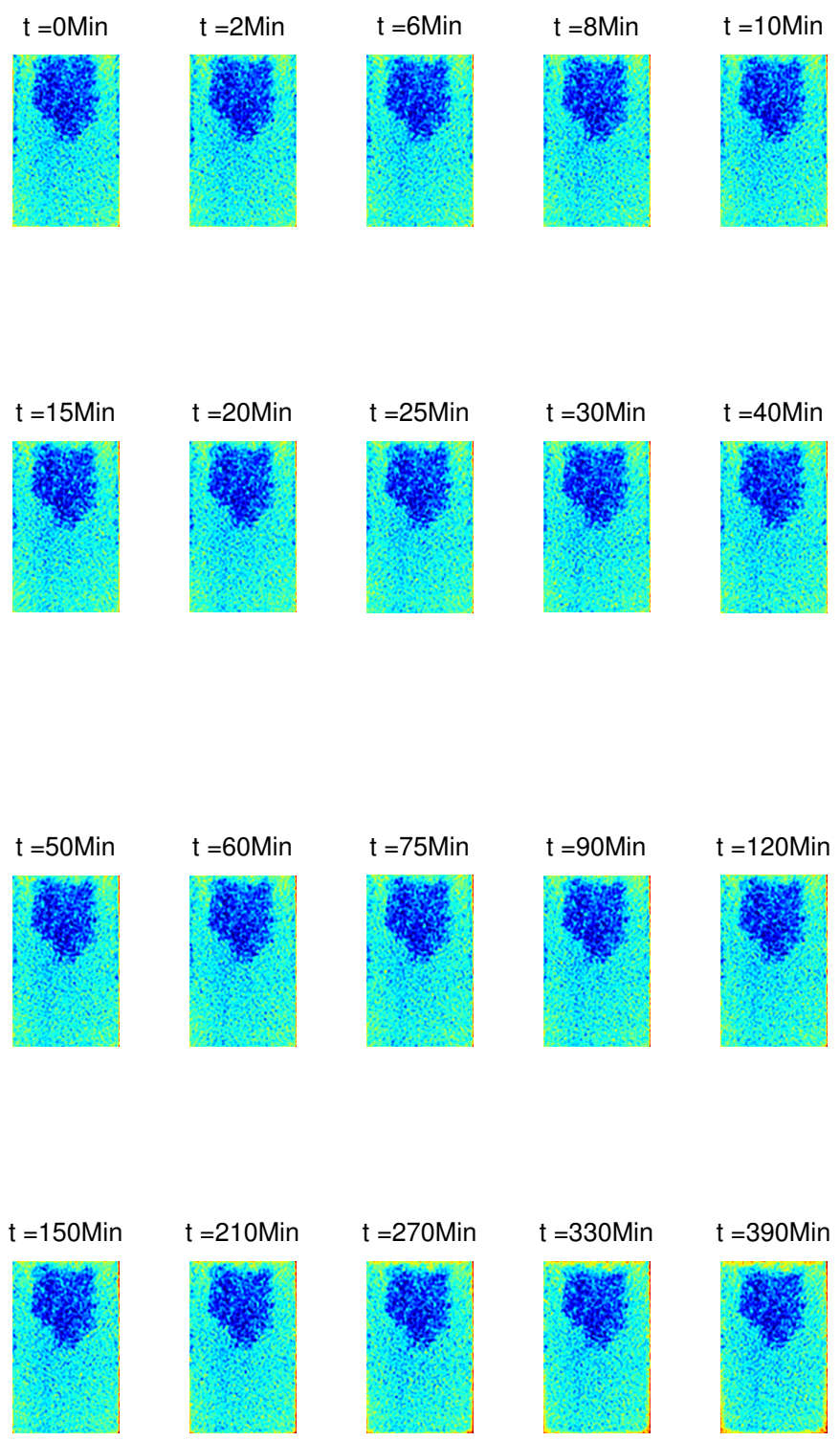


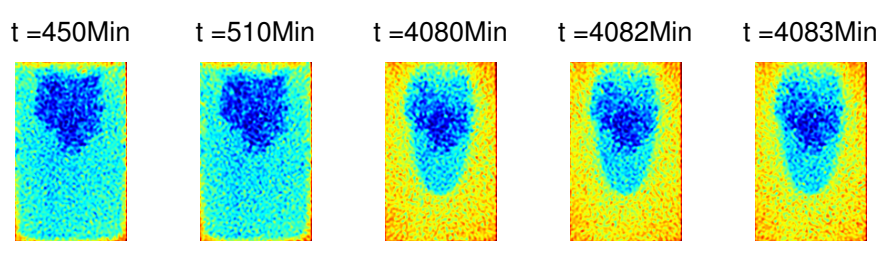

Figure 4.41: Visualization of penetration of Decalin from the fracture into the saturated core with Eicosane 


\subsection{Hexadecane-Pentane}

The last experiment was conducted using Pentane as slovent and Hexadecane as model oil. Pentane is much lighter than the previous solvent, Decalin. We did this experiment twice. Once we doped the solvent as previous and once the model oil was doped. In the first case we could not observe anything with the CT scanner, but the second one shows the changes. Following the results of this experiment are provided.

\subsubsection{Averaging the CT-numbers along the core}

Again for the first step the core is subdivided into the small sections and the average of the CT numbers are determined in each section. Figure 4.42 shows the variation of the CT numbers along the core with time.

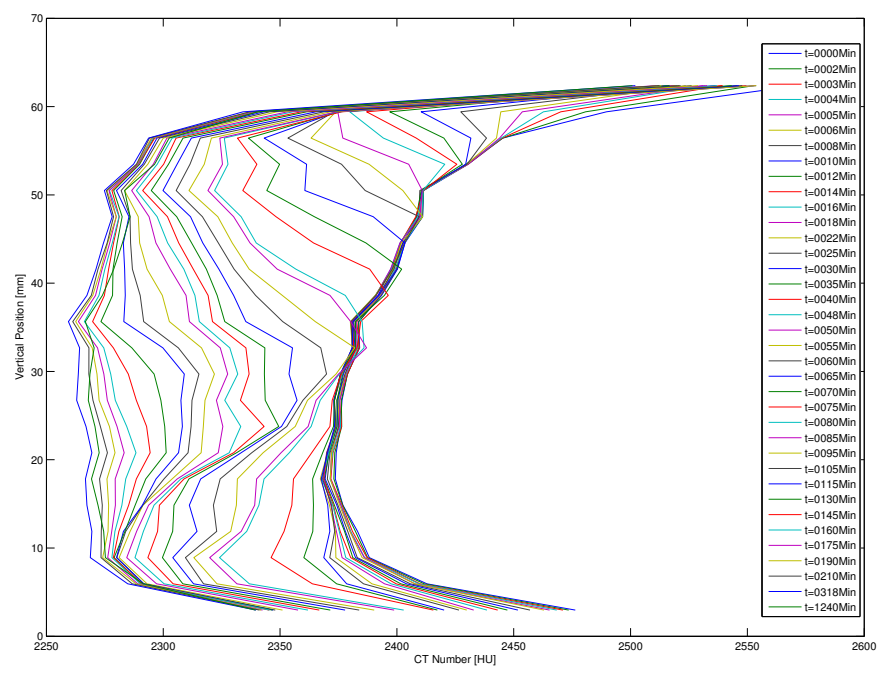

Figure 4.42: Averaged CT numbers along the core in different tims Hexadecane-Pentane system 
As can be seen the variation of the CT numbers do not follow a distinct pattern as the previous cases. CT numbers increase from the bottom and the top, but more from the top and gradually at the later time steps the variation of the $\mathrm{CT}$ numbers become uniform along the core.

\subsubsection{Saturation Calculation}

At this stage we calculate the saturation of the solvent inside the core using Eq.4.1. Figure 4.43 illustrates the saturation profile of the solvent along the core during the experiment.

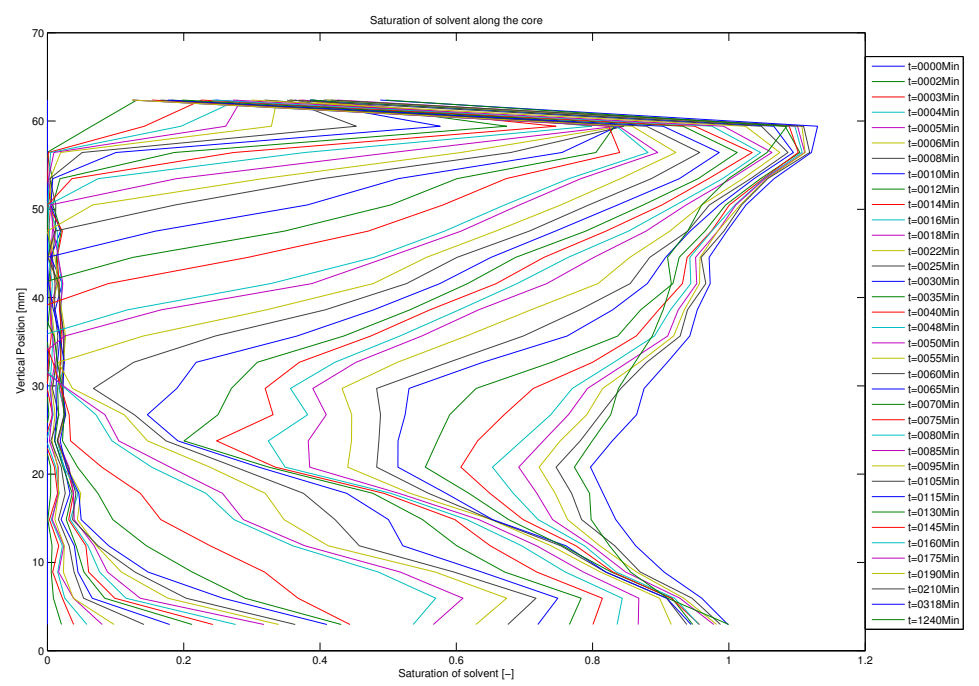

Figure 4.43: Variation of average of solvent saturation along the core in different time steps - Hexadecane-Pentane system 
As can be seen the saturation of solvent is not uniform along the core. The saturation of the solvent is more at the top of the core, which could be due to lower density of the Pentane. Additionally, because the viscosity of the Pentane is much lower than the viscosity of heexadecane the front is unstable.

\subsubsection{Recovery}

The recovery of Hexadecane could be calculated by assuming the conservation of fluids, using Eq.4.2. Recovery profile is produced by averaging the produced oil ( saturation of solvent) at different time step. Figure 4.44 illustrates the recovery of hexadecane from the matrix due to penetration of Pentane.

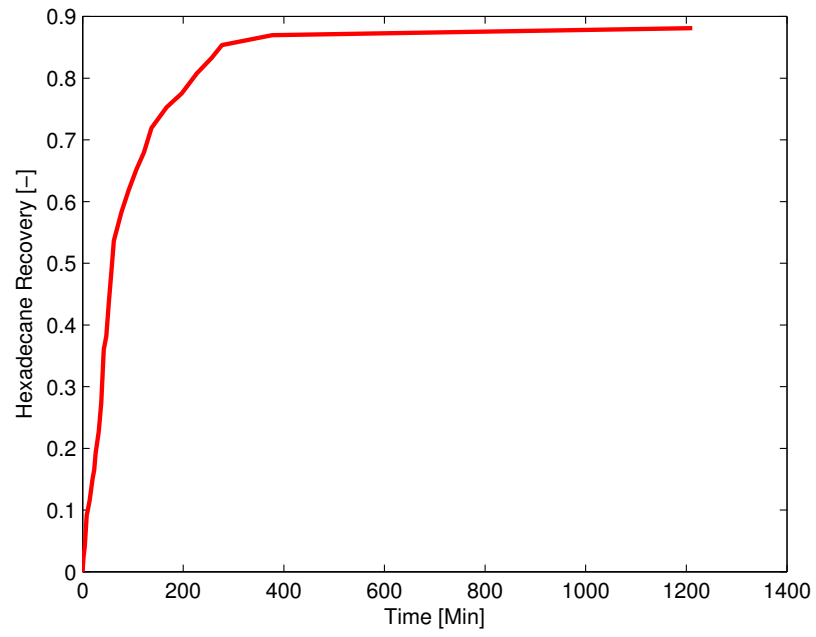

Figure 4.44: Recovery profile of Hexadecane 


\subsubsection{Visualization of the process}

At this stage we visualize the whole process. Following figures illustrate the difference of CT images at different time steps. Note that the orange color represents Hexadecane inside the core and green color is Pentane.

As can be seen Pentane starts to penetrate into the core from the top edge of the core after 14 minutes and then it expands inside the core gradually. The penetration of Pentane does not have a distinct pattern and it is unstable. The most likely reason for this behavior is lower viscosity of the solvent. 

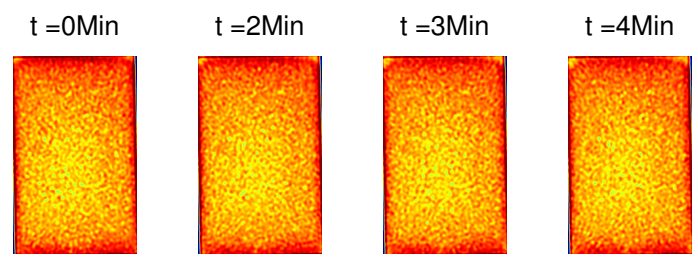

$\mathrm{t}=5 \mathrm{Min}$

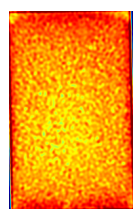

$\mathrm{t}=6 \mathrm{Min}$
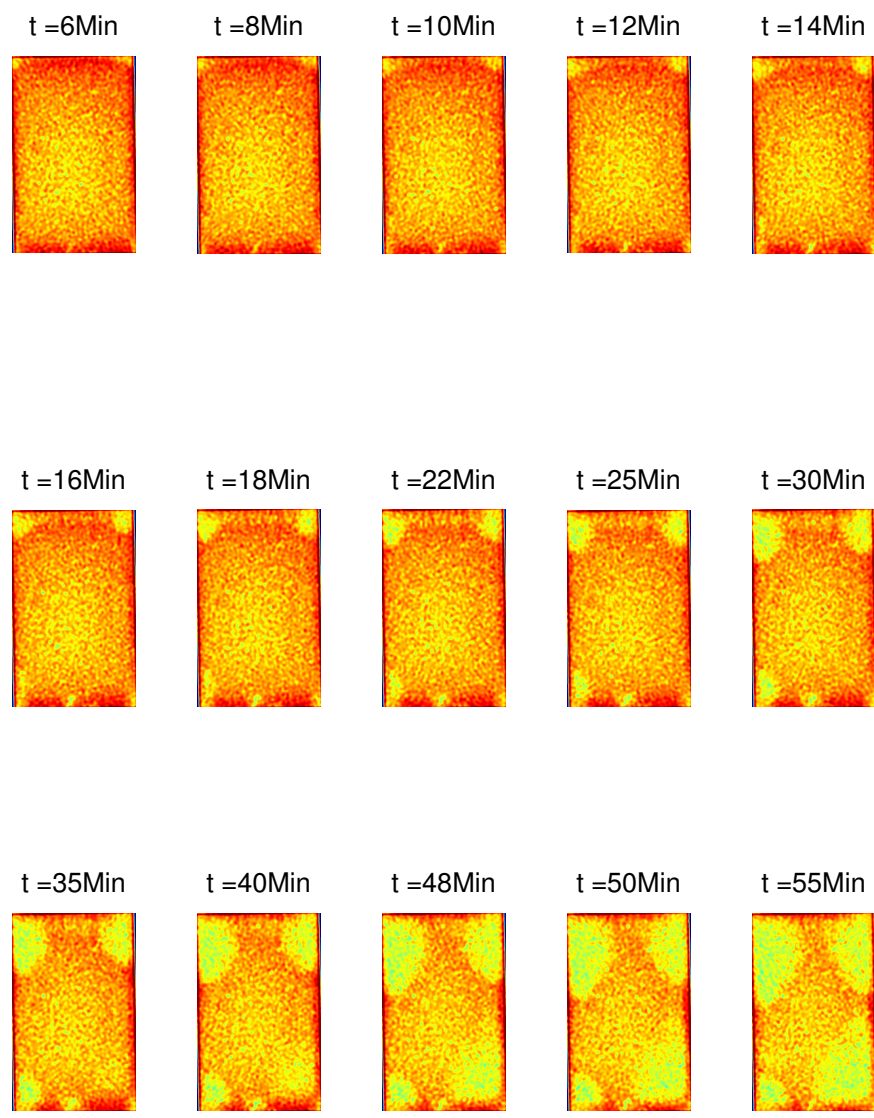

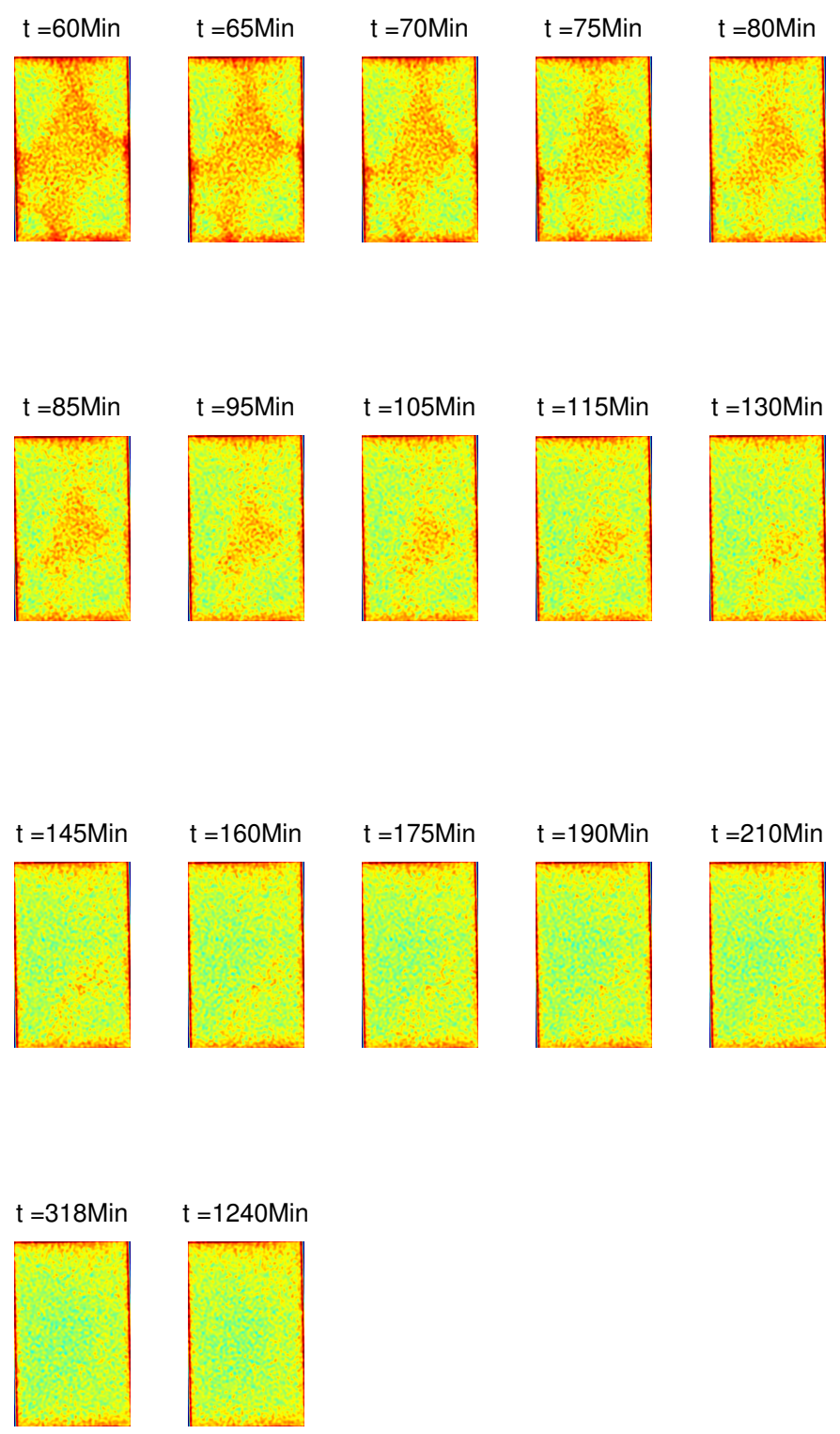

Figure 4.45: Visualization of penetration of Pentane from the fracture into the saturated core with Hexadecane 


\section{Chapter 5}

\section{Simulation}

\subsection{History matching of soak experiments}

The physical model and the governing equations of the problem are discussed in Chapter 2. In order to numerically reproduce the experimental data, The governing equations are solved with a finite difference method using the reservoir simulation software packages MoReS (Shell in-house simulator) to numerically reproduce the experimental data. The radial coordinates option was used and grid sizes are taken to be $1 \mathrm{~mm}$ in each $(\mathrm{R}, \mathrm{Z})$ direction. The numerical model describes fluid transport and mixing in a cylindrical core saturated with oil immersed in a jar filled with solvent. A spacer at the bottom of the jar keeps the core at a distance of $10 \mathrm{~mm}$ from the bottom. The geometry of the model is shown in Figure 5.1. The rock and fluid properties of the numerical model are identical to those of the experiment as mentioned in section 2. A permeability of $10000 \mathrm{D}$ and porosity of 1 was assigned to the fracture. Fig. 13 shows the R-Z cross sectional view of the

MoReS simulation model. Note that the area shown in green represents the core plug and red area represents the solvent in the fracture. 


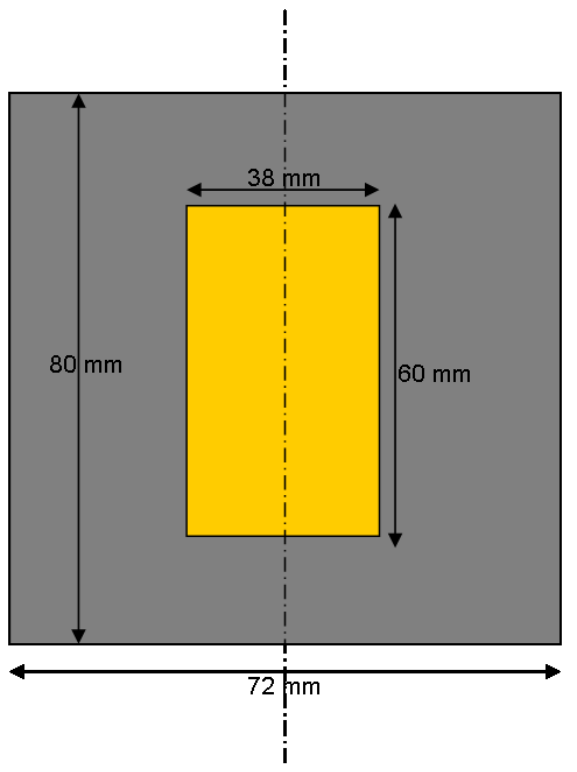

Figure 5.1: Geometry of the model

The flow is driven by gravity due to the spatial dependence of the density on the concentration. Also mixing due to diffusion plays a role.

Figure 5.2 shows the R-Z cross sectional view of the MoReS simulation model. Note that the area shown in green represents the core plug and red area represents the solvent in the fracture. 

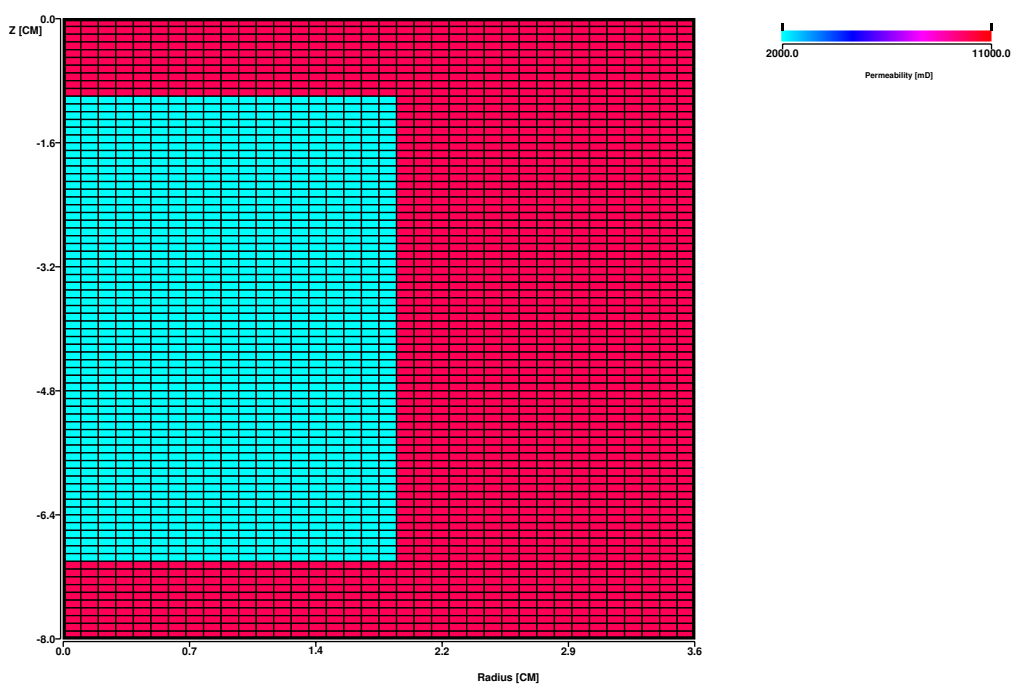

Figure 5.2: R-Z cross-sectional view of MoReS simulation model

Due to first contact miscibility of the model oil and the solvent, we employ two components (model oil and solvent) and single phase (oil) in our simulation model with density and viscosity mixing rules. A log-linear viscosity mixing and mass weighted inverse mixing rule were used for oil phase. Eq.5.1 shows the log linear viscosity mixing rule and the density mixing rule.

$$
\begin{aligned}
& \mu_{\text {mix }}=\mu_{\text {model oil }}^{\mathrm{C}_{\text {model }}} \times \mu_{\text {solvent }}^{\mathrm{C}_{\text {solvent }}} \\
& \rho_{\text {mix }}=\frac{1}{\left(\frac{X_{\text {model oil }}+\frac{X_{\text {solvent }}}{\rho_{\text {model oil }}}}{\rho_{\text {solvent }}}\right)}
\end{aligned}
$$

where $\mu$ is viscosity, $C$ stands for the concentration (mole fraction), $\rho$ is density and $X$ is the mass fraction and calculated as:

$$
X_{i}=\frac{C_{i} \times \mathrm{MW}_{i}}{\sum C_{i} \times \mathrm{MW}_{i}}
$$

where 'MW' is the molecular weight of the component. This mixing rule was validated by measuring the viscosity of different compositions of the model oils and solvents. 
Single phase flow is always assumed due to first contact miscibility. The model also includes gravity to capture effects caused by density differences between the two components.

In this study the history matching is obtained by varying the diffusion coefficient. The effective diffusion coefficient in a porous medium is different from bulk diffusion due to tortuosity and enhanced mixing effects in flowing fluids (see for example Perkins and Johnston (1963) and Bruining et al. (2012)). However, we will use the effective diffusion coefficient as a parameter, to obtain an optimal match between the numerical model and the experiment. Porous media can be considered as a network of flow chambers, having random size and flow conductivity, connected together by openings of smaller size. In such a system, the apparent diffusion coefficient is less than the molecular diffusion coefficient, as measured in the absence of a porous medium [Perkins and Johnston (1963)]. Consequently, the parameter which is used in the simulation to obtain a history match is an effective diffusion coefficient instead of molecular diffusion coefficient. Effective diffusion coefficient is related to the molecular diffusion coefficient through the degree of tortuosity of the pore system in the core material. Eq.5.3 shows the relation between the effective diffusion coefficient and the molecular diffusion coefficient.

$$
D_{e f f}=\frac{D_{m}}{F \varphi}=\frac{D_{m}}{\tau}
$$

where $D_{m}$ is the effective diffusion coefficient, $F$ is formation electrical resistivity, $\varphi$ is porosity, $D_{m}$ is the molecular diffusion coefficient and $\tau$ is the formation tortuosity.

In the next section the results of history matching for each experiments are provided.

\subsubsection{History match of experiment with Pentane-Decalin}

In order to numerically reproduce the experiment with pentane and decalin and obtain the history match, we assign an effective diffusion coefficient of $10^{-9} \mathrm{~m}^{2} / \mathrm{s}$ as a starting value, see also Cussler (2007) and Perkins and Johnston (1963). Then we vary the diffusion coefficient to match the recovery profile. Finally we get an acceptable match with a diffusion coefficient 
of $9 \times 10^{-9} \mathrm{~m}^{2} / \mathrm{s}$. Figure 5.3 illustrates the simulation results and its good agreement with experimental data. We used logarithmic scale to show the match in early time steps.

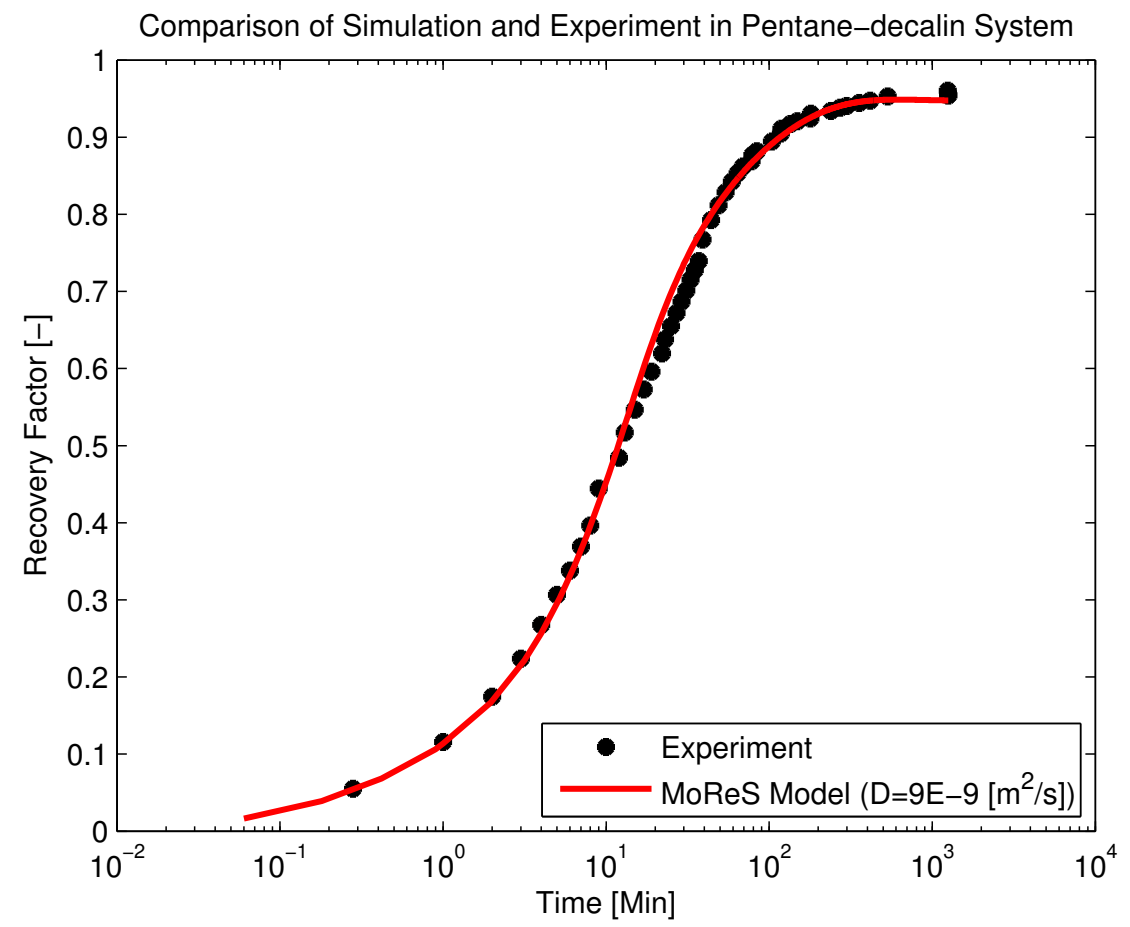

Figure 5.3: History match of soak experiment with pentane and decalin $\mathrm{D}=9 \mathrm{E}-9 \mathrm{~m}^{2} / \mathrm{s}$ 
In order to investigate that the displacement is governed by pure diffusion or by gravity, we conduct an additional simulation by neglecting gravitational forces. As can be seen from Figure 5.4, the recovery profile obtained with the gravity term in the model is different with the model that includes gravity term in early time steps.

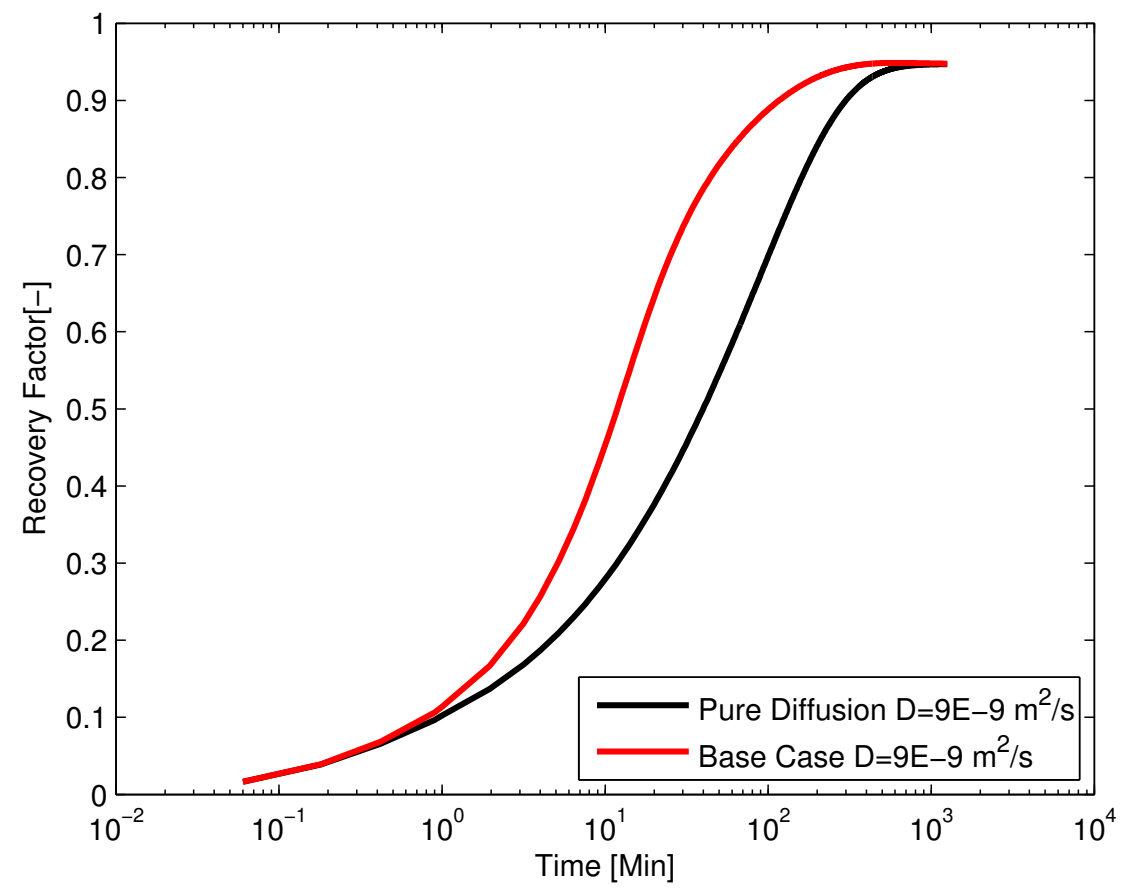

Figure 5.4: Simulation for the cases with and without gravitational forces for Pentane-Decalin system 


\section{Sensitivity Analysis}

As it is mentioned in Chapter 3, permeability of a Bentheimer Sandstone core can vary between $2 \mathrm{D}$ and $2.5 \mathrm{D}$. We conducted additional simulations to investigate the sensitivity of the problem to permeability changes. We varied the permeability from 1.5 D to $2.5 \mathrm{D}$ and compared the results. Figure 5.5 illustrates the results of sensitivity analyses with regards to the permeability changes.

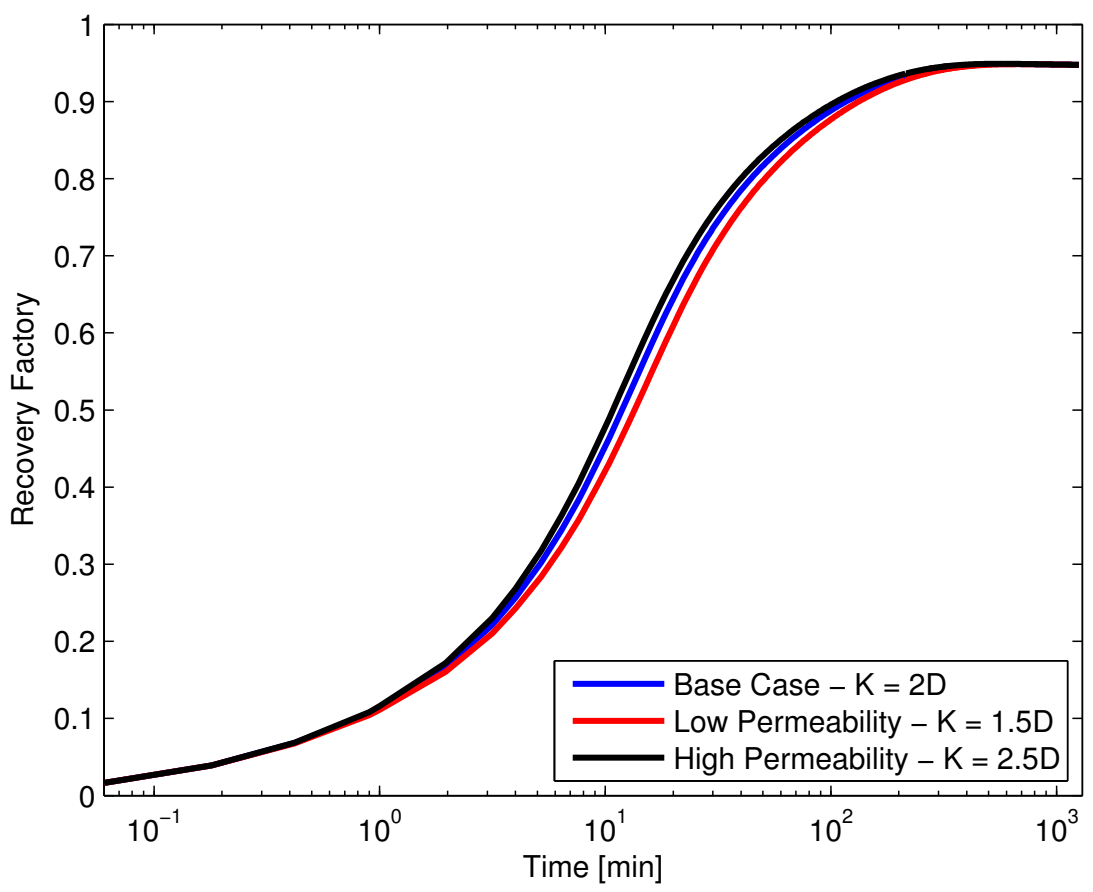

Figure 5.5: Sensitivity analysis of the simulation with respect to the permability 
As it can be seen in Figure 5.5 the simulation results do not change that much with varying the permeability, i.e., the simulation is not sensitive to the permeability.

We also conducted a set of simulations with varying grid block sizes (1 and $0.5 \mathrm{~mm}$ ) to validate the adequacy if $1 \mathrm{~mm}$ grid block size. As can be seen in Figure 5.6, results are in a good agreement.

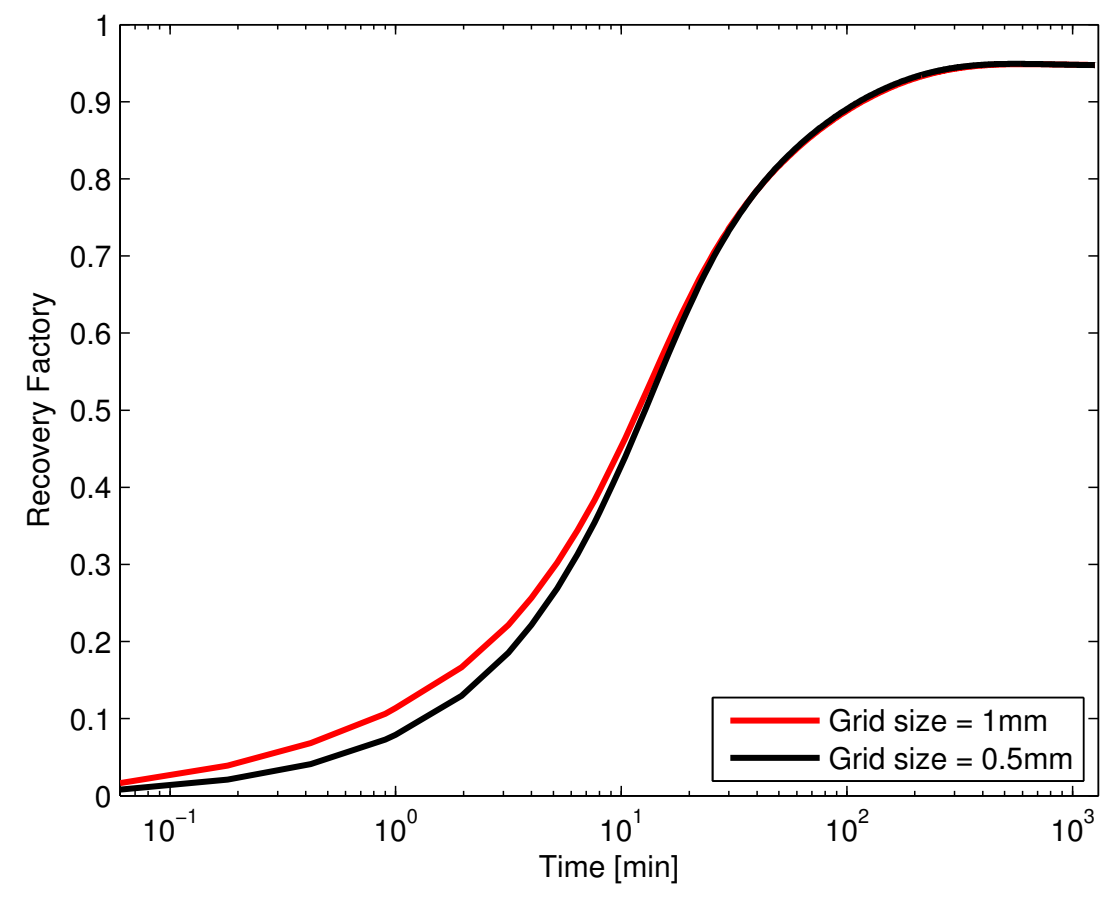

Figure 5.6: Predicted recovery profiles by varying the grid block sizes. Note that grid block size of $1 \mathrm{~mm}$ gives adequate results 


\subsubsection{History match of experiment with Hexadecane- Decalin}

Figure 5.7 compares simulation results with experimentally observed recovery factors in the hexadecane-decalin system. The starting value for diffusion coefficient is somewhat smaller due to the higher viscosity of the model oil. The best match is obtained by lowering the diffusion coefficient down to $10^{-11}$ $\mathrm{m}^{2} / \mathrm{s}$. This is much smaller than the diffusion coefficient calculated from the Wilke-Chang correlation (1955) $\left(0.35 \times 10^{-10} \mathrm{~m}^{2} / \mathrm{s}\right.$, which, in porous media, includes a tortuosity factor of 0.7) (see Bear (1972) and Bruining et al. (2012)). The discrepancy can be explained by the presence of numerical diffusion. Figure 5.7 illustrates the simulation results and its agreement with experimental data. Results are shown in logarithmic scale to show the match in early time steps. 


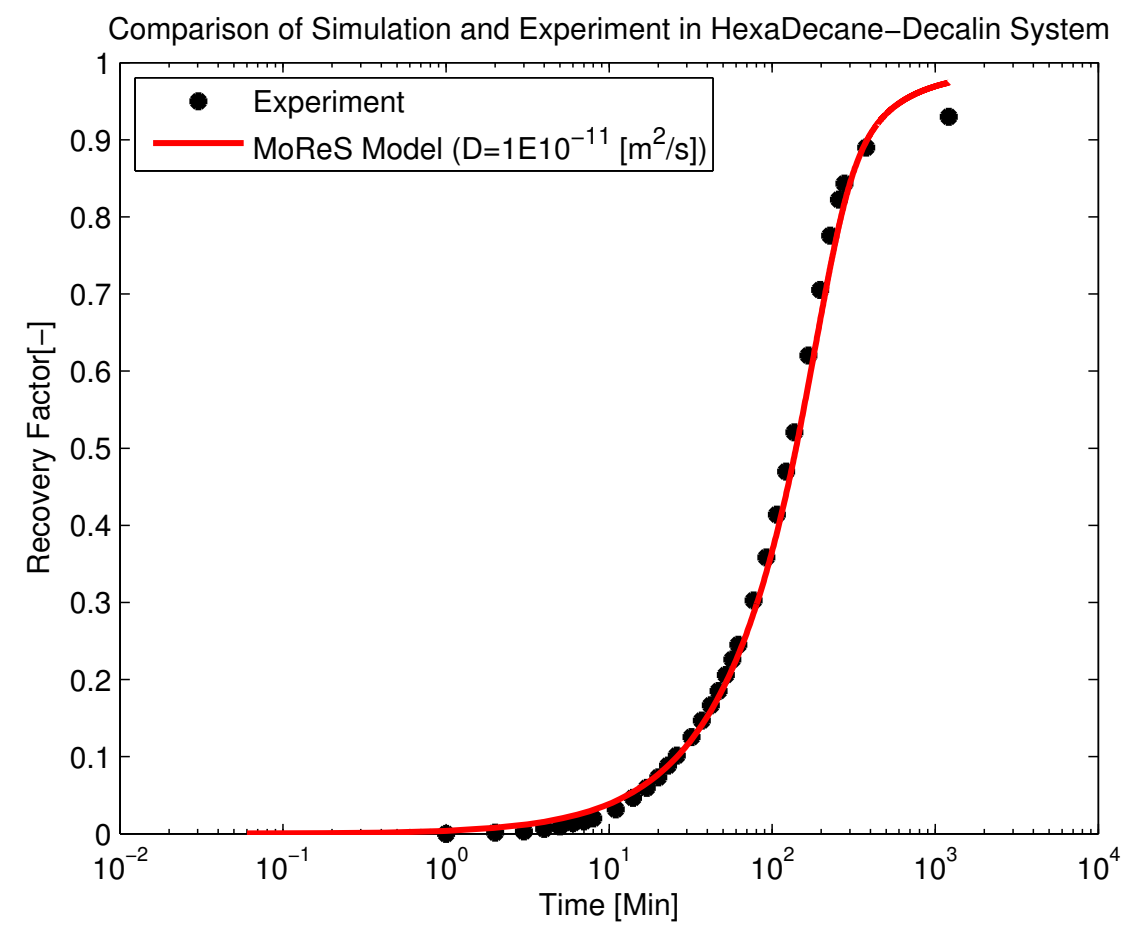

Figure 5.7: History match of soak experiment with hexadecane and decalin $\mathrm{D}=1 \mathrm{E}-11 \mathrm{~m}^{2} / \mathrm{s}$

As it is discussed in chapter 4 , the contour lines in concentration profile of decalin are no longer predominantly vertical along the core and this suggests that the displacement is predominantly influenced by gravity and less by diffusion. A simulation is conducted without including the gravitational forces to confirm this observation. It can be seen in the Figure 5.8 that the recovery profile for the case without the gravitational forces is much lower than the base case with gravitational forces. This discrepancy between these two cases is in line with the idea that the displacement process in the hexadecane-decalin system is governed by gravitational forces. 


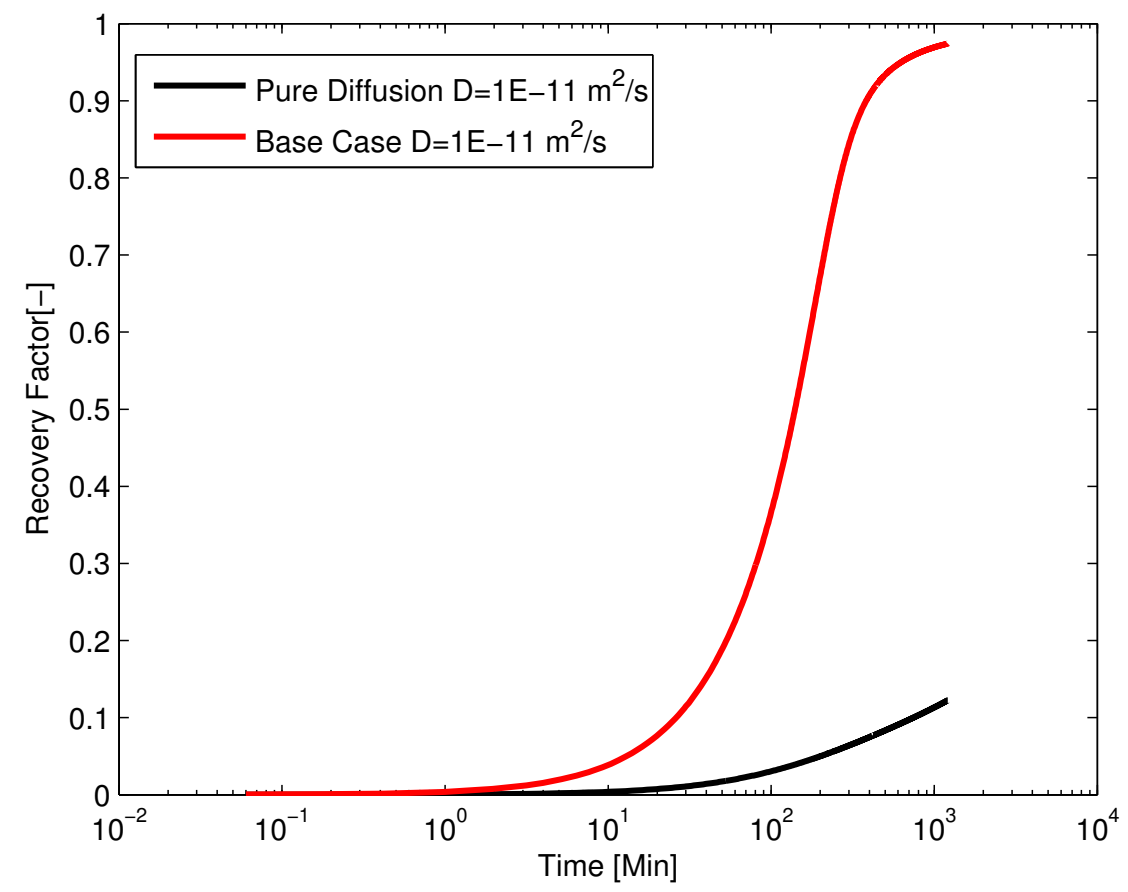

Figure 5.8: Simulation for the cases with and without gravitational forces for Hexadecane-Decalin system

\subsubsection{History match of experiment with Hexadecane- Pentane}

Figure 5.9 shows the recovery profile of the hexadecane-pentane system obtained from the simulation and the experiment. There is a good agreement between the experiment and the simulation, except at the end of the experiment. The reason for the small diffusion coefficient that gives the optimal match is explained above for simulation 2. The discrepancies towards the end of the experiment could be due to the assumption of radial symmetry, which can be destroyed due to natural convection effects. All data are representative for a central slice of the core, which in case of radial symmetry would be also representative for the full 3-D profile. Natural convection, however, may destroy this symmetry, and therefore there is also no symmetry in the production of hexadecane from the core; i.e., the production could be different 
in each slice of the core.

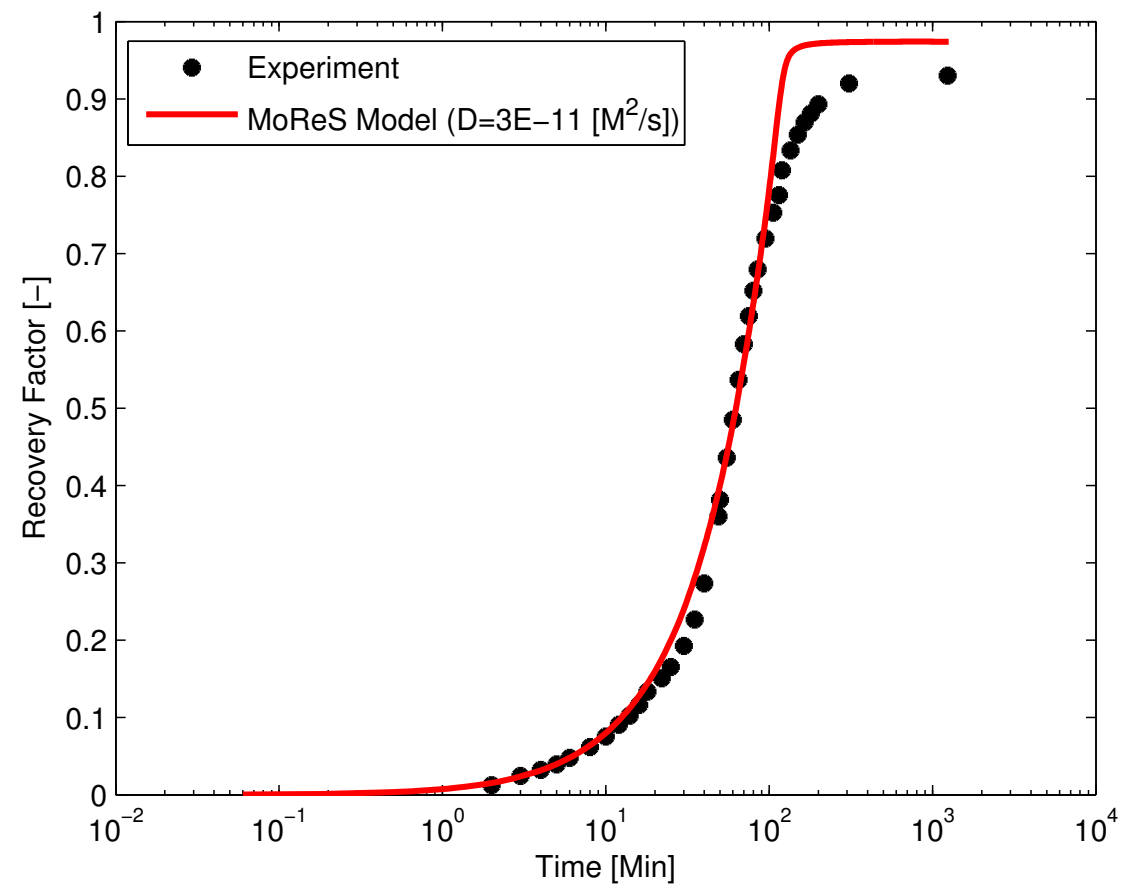

Figure 5.9: History match of soak experiment with hexadecane and decalin $\mathrm{D}=3 \mathrm{E}-11 \mathrm{~m}^{2} / \mathrm{s}$ 


\section{$5.2 \quad$ Scaling Numbers}

With proper definition of the time-scale for each key mechanism (gravity and diffusion), the ratio of diffusion time $\left(t_{\text {diff }}\right)$ to gravity time $\left(t_{\text {grav }}\right)$ becomes an important parameter controlling how fast the oil in a matrix block can be recovered by solvent penetration. In order to derive the scaling numbers we need to specify the characteristic lengths for diffusion and gravity drainage. The main diffusion mixing occurs perpendicular to the fracture, i.e., in the radial direction that contains the largest concentration gradients. The characteristic length is the core radius. Density variations cause natural convection in the fracture system, causing fresh solvent at the fracture-matrix boundary. The gravity leads to flow in the axial direction and hence the characteristic length is the height of the sample.

Therefore, we define two characteristic times, viz., the diffusion and the gravity time to characterize the experimental data. These numbers are used to classify the relative importance of the diffusion process and the gravity-driven convection mass transfer between the fracture and matrix in the conducted experiments.

The diffusion time is defined as the time required for a component to diffuse over a distance of the order of the radius $r$ of the cylinder

$$
\mathrm{t}_{\mathrm{diff}}=\frac{r^{2}}{\mathrm{D}}
$$

while the gravity time is defined as the time a fluid element needs to transverse from the bottom to the top (or from top to bottom) of the core. It is given by the quotient of the height of the cylinder and the gravity driven Darcy velocity

$$
\mathrm{t}_{\text {grav }}=\frac{\mu_{\text {solvent }} \mathrm{h}}{\mathrm{k}\left(\rho_{\text {solvent }}-\rho_{\text {oil }}\right) g}
$$

where $r$ is the core radius, $K$ is the permeability, $\rho$ is the density, $g$ is the gravity, $D$ is the effective diffusion coefficient, $\mu$ is viscosity and $h$ is the vertical height. $t_{\text {diff }}$ is the time required for a solvent to recover significant amount of oil from a matrix block via diffusion only. $t_{\text {grav }}$ represents how long the mobilized oil stays in the matrix. A smaller $t_{\text {grav }}$ is better for the recovery process as it implies a faster circulation of fresh solvent into the matrix and 
creating a sharper concentration front to diffuse more solvent into the matrix.

In these equations $D$ is the diffusion coefficient. For calculating the diffusion coefficient we use the Wilke-Chang (1955) equation (see also Poling and Prausnitz (2001)), which is in essence, an empirical modification of the Stokes-Einstein relation and which reads,

$$
D_{m}=\frac{7.4 \times 10^{-8}\left(\Phi M_{s}\right)^{0.5} T}{\mu_{o} V_{s}^{0.6}}
$$

where $M_{s}$ is the molecular weight of solvent, $T$ is the temperature, $\mu_{o}$ is the viscosity of the model oil, $V_{s}$ is the molar volume of the solvent and $\Phi$ is the association factor of the model oil, which we take equal to 1 as Wilke and Chang (1955) recommended for unassociated materials. Note that this equation predicts the molecular diffusion coefficient, while in the simulations we use effective diffusion coefficient $(D)$ to model the experiments.

Calculating the characteristic time we obtain,

Table 5.1: Gravity and Diffusion time and $\mathrm{N}_{\mathrm{GD}}$

\begin{tabular}{|cccc|}
\hline \hline Parameter & Pentane-Decalin & Hexadecane-Decalin & Hexadecane-Pentane \\
\hline \hline $\mathrm{T}_{\text {grav }}[\mathrm{s}]$ & $0.27 \times 10^{4}$ & $8.4 \times 10^{4}$ & $7.5 \times 10^{4}$ \\
\hline $\mathrm{T}_{\text {diff }}[\mathrm{s}]$ & $10.3 \times 10^{4}$ & $78.9 \times 10^{4}$ & $60.5 \times 10^{4}$ \\
\hline \hline
\end{tabular}

The experiments can therefore be classified according to these numbers. These numbers, however, indicate the initial behaviour and are only qualitative. The pentane-decalin system is characterized by fast diffusion and even faster gravity drainage. The hexadecane-decalin system is characterized by very slow diffusion, and also slow gravity drainage. In this case we observe only gravity drainage and negligible diffusion effects. The hexadecanepentane system has initially almost the same characteristic values as the hexadecane-decalin system. However the behavior is completely different. This is possibly related to the fact that the final numbers are more dominated by the presence of pentane leading to faster diffusion and gravity drainage times. 


\section{Chapter 6}

\section{Summary and Conclusions}

Conducting a series of miscible-displacement experiments in porous rocks has tested the performance of a solvent as a displacing fluid for enhancing the oil recovery. The rock has been initially saturated by a model oil, which is then immersed in a solvent to study the production mechanism. Pentane, Hexadecane and Eicosane were used as oils spanning a large range of densities and viscosities. Decalin and Pentane were used as a heavy solvent and light solvent respectively. In most of the experiments, the dominating feature of the displacement was gravity-enhanced displacement.

The 2D spatial distribution of the penetration of the solvent and the displaced oils were obtained using X-ray computed tomography (CT-scanning). Oil recovery and saturation profiles were obtained from CT and compositional data. The experimentally-obtained-oil-recovery curves were compared to the numerical model. The numerical model considers (natural) convection and diffusion as transfer mechanisms and employs single-phase Darcy's law to calculate velocities. The viscosity and the density of the mixture of the solvent and the oil were determined by mixing rules for miscible fluids.

The following conclusion can be drawn from the experimental and the simulation results:

- CT-scanning can be used to visualize the interaction between the fracture and the matrix and quantify the recovery and concentration profiles of gravity enhanced miscible displacement. The importance of the mechanisms involved can be studied by experimenting with various light and heavy hydrocarbons representing different oils and solvents. 
- In case of a low-density difference between solvent and oil, a doping agent should be added to one of the component to increase the CTcontrast.

- Although it would be better to add the doping agent to the component that we want to trace (solvent) during the experiment but in case of lighter solvent the doping agent should be added to the model oil.

- At low oil (pentane) viscosity and high solvent (decalin) viscosity both diffusion and gravity forces are important and the recovery process is fast. The CT scan results show that the solvent penetrates into the core from the sides and the bottom of the core almost equally. There is no penetration from the top.

- At higher oil (hexadecane) viscosity the recovery process is dominated by gravity. The CT scan results show that in this case solvent penetrates into the core exclusively from the bottom.

- At very high oil viscosity, the recovery process is very slow and is governed mainly by diffusion.

- When the viscosity of the solvent (pentane) is lower than the viscosity of the oil (hexadecane), the diffusion process leads to an unstable displacement of the oil by the solvent. Although, in this case the solvent penetrates into the core more from the top, the penetration of the solvent does not follow a special pattern. The CT scan results show the penetration from all sides of the core.

- It is possible to simulate the recovery profiles, using MoReS. The diffusion coefficients used to obtain an optimal match between the experimental data and the model are unrealistically low, which can be explained by numerical diffusion. The simulation results of the low permeability core were too preliminary to include in the paper.

\section{Recommendations}

The following recommendations can be considered for the further studies:

1. Study the effect of the numerical dispersion 
2. Conducting experiments with high viscosity oil and low permeable rock

3. Conducting a 3D experiment for an unstable oil displacement

4. Study the effect of the pressure and the temperature

5. Upscaling of the experiments

6. Study the effect of the doping agent on the fluid properties.

7. Viscosity measurements of different compositions of Eicosane and Decalin for sake of numerical modeling of Eicoase-Decalin experiement 


\section{Bibliography}

[1] B. Adibhatla and K. Mohanty. Oil recovery from fractured carbonates by surfactant-aided gravity drainage: laboratory experiments and mechanistic simulations. In SPE/DOE Symposium on Improved Oil Recovery, 2006.

[2] H. Al-Hadhrami and M. Blunt. Thermally induced wettability alteration to improve oil recovery in fractured reservoirs. SPE Reservoir Evaluation E3 Engineering, 4(3):179-186, 2001.

[3] A. Andrianov, R. Farajzadeh, M. Mahmoodi Nick, M. Talanana, and P. Zitha. Immiscible foam for enhancing oil recovery: Bulk and porous media experiments. In SPE Enhanced Oil Recovery Conference, 2011.

[4] J. Bear. Dynamics of fluids in porous media. Dover publications, 1988.

[5] P. Belery. Molecular diffusion in naturally fractured reservoirs: A decisive recovery mechanism. In SPE Annual Technical Conference and Exhibition, 1989.

[6] S. Berg, S. Oedai, AJ Landman, N. Brussee, M. Boele, R. Valdez, and K. van Gelder. Miscible displacement of oils by carbon disulfide in porous media: Experiments and analysis. Physics of Fluids, 22:113102, 2010.

[7] DM Boersma and J. Hagoort. Displacement characteristics of nitrogen flooding vs. methane flooding in volatile oil reservoirs. SPE Reservoir Engineering, 9(4):261-265, 1994.

[8] H. Bruining, M. Darwish, and A. Rijnks. Computation of the longitudinal and transverse dispersion coefficient in an adsorbing porous medium using homogenization. Transport in Porous Media, pages 1-27, 2009. 
[9] AC Correa, KK Pande, and WE Brigham. Computation and interpretation of miscible displacement performance in heterogeneous porous media. SPE Reservoir Engineering, 5(1):69-78, 1990.

[10] E.L. Cussler. Diffusion: Mass transfer in fluid systems. Cambridge Univ Pr, 1997.

[11] G. Darvish, E. Lindeberg, T. Holt, J. Kleppe, and S. Arild Utne. Reservoir conditions laboratory experiments of $\mathrm{co} 2$ injection into fractured cores. In SPE Europec/EAGE Annual Conference and Exhibition, 2006.

[12] B. Dindoruk and A. Firoozabadi. Crossflow in fractured/layered media incorporating gravity, viscous, and phase behavior effects: Part iifeatures in fractured media. In SPE/DOE Improved Oil Recovery Symposium, 1996.

[13] B. Dindoruk and A. Firoozabadi. Crossflow in fractured/layered media incorporating gravity, viscous, and phase behavior effects: Part iformulation and features in layered media. SPE Journal, 2(2):120-135, 1997.

[14] V. Er and T. Babadagli. Miscible interaction between matrix and fracture: A visualization and simulation study. SPE Reservoir Evaluation ES Engineering, 13(1):109-117, 2010.

[15] R. Farajzadeh, A. Andrianov, and PLJ Zitha. Investigation of immiscible and miscible foam for enhancing oil recovery. Industrial \& Engineering Chemistry Research, 49(4):1910-1919, 2009.

[16] R. Farajzadeh, L.B. Wassing, and P. Boerrigter. Foam assisted gas oil gravity drainage in naturally-fractured reservoirs. In SPE Annual Technical Conference and Exhibition, 2010.

[17] F. Farshbaf Zinati, R. Farajzadeh, and P. Zitha. Modeling and ct scan study of the effect of core heterogeneity on foam flow for acid diversion. In European Formation Damage Conference, 2007.

[18] SA Farzaneh, R. Kharrat, and MH Ghazanfari. Experimental study of solvent flooding to heavy oil in fractured five-spot micro-models: The role of fracture geometrical characteristics. Journal of Canadian Petroleum Technology, 49(3):36-43, 2010. 
[19] A. Firoozabadi. Recovery mechanisms in fractured reservoirs and field performance. Journal of Canadian Petroleum Technology, 39(11), 2000.

[20] A. Firoozabadi, T. Markeset, and B. Dindoruk. Viscous displacement in fractured porous media. Journal of Canadian Petroleum Technology, 36(9), 1997.

[21] C.U. Hatiboglu and T. Babadagli. Diffusion mass transfer in miscible oil recovery: visual experiments and simulation. Transport in Porous Media, 74(2):169-184, 2008.

[22] H. Hoteit and A. Firoozabadi. Numerical modeling of diffusion in fractured media for gas injection and recycling schemes. In SPE Annual Technical Conference and Exhibition, 2009.

[23] H. Karimaie and O. Torsaeter. Low ift gas-oil gravity drainage in fractured carbonate porous media. Journal of Petroleum Science and Engineering, 70(1-2):67-73, 2010.

[24] J.E. Ladron De Guevara, F. Rodriguez-De La Garza, and A. GalindoNava. Gravity drainage and oil reinfiltration modeling in naturally fractured reservoir simulation. In International Oil Conference and Exhibition in Mexico, 2009.

[25] Y. Le Gallo, B. Bourbiaux, and G. Fernandes. Mass transfer in fractured reservoirs during gas injection: Experimental and numerical modeling. In SPE Annual Technical Conference and Exhibition, 1997.

[26] E. Lewis, E. Dao, and K. Mohanty. Sweep efficiency of miscible floods in a high-pressure quarter-five-spot model. SPE Journal, 13(4):432-439, 2008.

[27] S. H. Midtlyng and J. A. Stensen. Improved flooding experiments in heterogeneous rocks using in situ saturations from X-ray CT measurements. Society of Core Analysts, 2000.

[28] D. Morel, B. Bourbiaux, M. Latil, and B. Thiebot. Diffusion effects in gasflooded light-oil fractured reservoirs. SPE Advanced Technology Series, 1(2):100-109, 1993. 
[29] F.M. Orr Jr. Theory of gas injection processes. Tie-Line Publications, Copenhagen, Denmark, 2007.

[30] F. Perez-Cardenas, J. Cruz-Hernandez, and C. Perez-Rosales. Miscible displacement in fractured porous media. Paper SPE, 20798, 1990.

[31] TK Perkins and OC Johnston. A review of diffusion and dispersion in porous media. Old SPE Journal, 3(1):70-84, 1963.

[32] B.E. Poling, J.M. Prausnitz, and O.C. John Paul. The properties of gases and liquids, volume 5. McGraw-Hill New York, 2001.

[33] AM Saidi. Simulation of naturally fractured reservoirs. In SPE Reservoir Simulation Symposium, 1983.

[34] H. Salimi and J. Bruining. The influence of wettability on oil recovery from naturally fractured oil reservoirs including non-equilibrium effects. In SPE Latin American and Caribbean Petroleum Engineering Conference, 2010.

[35] M. Simjoo, M. Mahmoodi Nick, and P. Zitha. Effect of oil saturation on foam for acid diversion. In 8th European Formation Damage Conference, 2009 .

[36] KS Sorbie, RMS Wat, AO Hove, V. Nilsen, and J. Leknes. Miscible displacements in heterogeneous core systems: Tomographic confirmation of flow mechanisms. In SPE International Symposium on Oilfield Chemistry, 1989.

[37] E. Stalgorova and T. Babadagli. Modeling miscible injection in fractured porous media using non-classical simulation approaches. In SPE Russian Oil and Gas Conference and Exhibition, 2010.

[38] JW Strutt and L. Rayleigh. Investigation of the character of the equilibrium of an incompressible heavy fluid of variable density. Proc. London Math. Society, 14:170-177, 1883.

[39] G.Q. Tang and A.R. Kovscek. High-resolution imaging of high mobility ratio, immiscible displacements. In Proceedings of the Society of Core Analysts International Symposium (Society of Core Analysts, Dublin, CA, 2005), SCA2005-12. 
[40] G. Taylor. The instability of liquid surfaces when accelerated in a direction perpendicular to their planes. i. Proceedings of the Royal Society of London. Series A, Mathematical and Physical Sciences, pages 192-196, 1950.

[41] BM Thiebot and SS Sakthikumar. Cycling fractured reservoirs containing volatile oil: laboratory investigation of the performance of lean gas or nitrogen injection. Middle East Oil Show, 1991.

[42] JJ Trivedi and T. Babadagli. Efficiency of diffusion controlled miscible displacement in fractured porous media. Transport in Porous Media, 71(3):379-394, 2008.

[43] M. Verlaan and P. Boerrigter. Miscible gas-oil gravity drainage. In First International Oil Conference and Exhibition in Mexico, 2006.

[44] SL Wellington and HJ Vinegar. X-ray computerized tomography. Journal of Petroleum Technology, 39(8):885-898, 1987.

[45] CR Wilke and P. Chang. Correlation of diffusion coefficients in dilute solutions. AIChE Journal, 1(2):264-270, 1955.

[46] EM Withjack and I. Akervoll. Computed tomography studies of 3-d miscible displacement behavior in a laboratory five-spot model. In $S P E$ Annual Technical Conference and Exhibition, 1988.

[47] P. Zitha, P. Zitha, and M. Uijttenhout. Carbon dioxide foam rheology in porous media: a ct scan study. In SPE International Improved Oil Recovery Conference in Asia Pacific, 2005. 


\section{Appendix A}

\section{Theoretical Background}

\section{A.1 Gravity Drainage and diffusion}

In a matrix-fractured system, oil is drained from matrix due to density difference between oil in matrix and gas in fracture when the matrix is surrounded by gas in fracture. This mechanism is called gas oil gravity drainage. Gravity drainage is a recovery process in which gravity acts as the main driving force and where gas replaces the voidage volume. In the other word, it is a gas/oil displacement in which gravity forces are dominating [J. Hagoort]. Gravity drainage is a critical process taking place in naturally fractured reservoirs as it played a major role in the oil recovery. Different parameters affect GOGD process such as [Mudit Chordia et al.]:

- size and permeability of matrix block

- type of gas and oil

- temperature and pressure condition

- fracture size

- rate of gas flowing in the fracture

Depending on which parameter influences the process, diffusion plays a role. Gravity drainage happens when the gravitational forces exceed the capillary forces in a matrix surrounded by gas. According to Darvish et al studies, diffusion, oil swelling and gravity drainage were cited as governing mechanisms. 
In a permeable oil saturated matrix block surrounded by gas in fractures density differences dominates the drainage of oil from a matrix, but in case of low permeability and small size matrix blocks with high capillary pressure, diffusion plays the governing role.

In case of low permeability and under the influence of a gradient in chemical compositions or in the absence of a significant pressure gradient the relevant phenomenon is molecular diffusion. Consequently, the mass transfer between matrix and fracture due to diffusion plays the major role in recovery from naturally fractured reservoirs.

\section{A.2 Miscible Displacement}

When two fluids mix in all portions and their mixture remain a single phase, they are called first-contact miscible [J. Tan et al.].

In naturally fractured reservoirs, diffusion and convection cause the transfer between fluid flowing in fracture and original matrix fluid if they are miscible. A common assumption made for miscible interaction between fracture and matrix is that fluid flow arising from pressure gradients and gravity occurs only through the fracture network and fluid in the matrix is assumed to be non-flowing but mass transfer of dissolved species may occur by molecular diffusion according to Ficks law.

In the directly miscible case the counter-current flow involves the countercurrent flow of components. Co-current aspects are also possible when the net flow direction is from one side of the matrix to the other. Counterand co-current processes comprise diffusion, natural convection and gravity drainage.

\section{A.3 Diffusion}

Diffusion describes the spread of particles through random motion from regions of higher concentration to regions of lower concentration. Diffusion is caused by random molecular motion that leads to complete mixing. Diffusion could be a slow process, in gases, diffusion progresses at a rate of $5 \mathrm{~cm} / \mathrm{min}$, 
in liquids it is about $0.05 \mathrm{~cm} / \mathrm{min}$ and in solids its rate may be only about $0.00001 \mathrm{~cm} / \mathrm{min}$ [E.L. Cussler]. The description of diffusion involves a mathematical model based on a fundamental hypothesis or law. There are two common choices fir such a law: one which uses diffusion coefficient and one involves a mass transfer coefficient. Fick's law of diffusion, uses a diffusion coefficient.

\section{A.3.1 Fick's Law}

Fick's first law relates the diffusive flux to the concentration. Fick defined a total one-dimansional flux as

$$
j=-D \frac{\partial c}{\partial z}
$$

where $j$ is the diffusion flux per unit area per unit time $\left[\frac{\text { amountofsubstance }}{\text { length }^{2} . t i m e}\right]$, $D$ is the diffusion coefficient $\left[\frac{\text { length }}{\text { time }}\right], c$ is the concentration in dimension of $\left[\frac{\text { amountofsubstance }}{\text { length }^{3}}\right]$ and $z$ is the position [length].

Fick's second law predicts how diffusion causes the concentration to change with time and it is expressed as follows in two dimensions,

$$
\frac{\partial c}{\partial t}=D \nabla^{2} c
$$

To have an idea about the values of diffusion coefficients it should be mentioned that the diffusion coefficient in gases can be estimated theoretically about $10^{-5} \mathrm{~m}^{2} / \mathrm{sec}$. Diffusion coefficients in liquids are around $10^{-9} \mathrm{~m}^{2} / \mathrm{sec}$ and diffusion coefficients in solids are slower still, around $10^{-34}$ $\mathrm{m}^{2} / \mathrm{sec}$. [book] 


\section{Appendix B}

\section{Principals of Computerized Tomographic Imaging}

\section{Introduction}

X-ray computed tomography $(\mathrm{CT})$ is a medical imaging method employing tomography created by computer processing. CT scanners produce cross sectional images by measuring the attenuation of a beam of X-ray which is rotating around the object. These sets of measurements are used to make a cross sectional image by using Fourier transforms algorithms, i.e. images of cross sections of object are produced by measuring the attenuation of x-rays along a large number of lines through the cross section. The basic concept of tomographic imaging is reconstructing an image from its projections. A projection is the integral of the image in the direction of a specific angle. The output of a CT scanner is matrices which contains attenuation coefficient of the scanned material for a specific effective energy. CT scanners do not have the medical usage only and in recent years they found large variety of application such as petroleum engineering. CT scanning is a non-destructive technique for visualizing the dynamic process and static structure in an object. Hence they are used for imaging different parameters and mechanisms in petroleum engineering. Image processing and image reconstruction in CT scanners is a controversial subject because it introduces noises and artefacts. Investigation of the image processing methods in CT scanners and the algorithms behind those could help us for a better perception of reliability of the analysis which are based on CT data. 


\section{B.1 Theory}

In a CT scanner the source and the detector rotate together around an object. As the X-ray source rotates around an object the beams penetrate the object at different angle and detectors record the transmitted X-ray intensity. In this manner many different $\mathrm{X}$-ray attenuations are recorded for mathematical reconstruction. What is measured in each volume element (voxel) is the linear attenuation coefficient which is defined by Beers law:

$$
\frac{I}{I_{0}}=\exp ^{-\mu h}
$$

where the variables are defined as follows:

$I$ : intensity remaining after the X-ray passes through a thickness $h$ of homogenous sample

$I_{\mathbf{0}}$ : incident X-ray intensity

$\boldsymbol{\mu}$ : linear attenuation coefficient If the sample is heterogeneous then the attenuation coefficient will be different in various dimensions and energy transmitted along the path length from source to detector $(L)$ is defined as follow:

$$
\ln \left(\frac{I}{I_{0}}\right)=\int_{0}^{L} \mu(h(x, y)) d h
$$

where $h(x, y)$ are the co-ordinates of the attenuation coefficient in $x$ and $y$ dimensions.

The X-rays penetrate a thin volumetric slice of object (voxel), and entire detector reading for a number of source $\backslash$ detector positions can be represented as a sinogram. The intensities in the sonogram are proportional to the line integrals of the attenuation coefficient of the object between source and detector. With the techniques of image reconstruction a two-dimensional image of the attenuation coefficient distribution in the object can be produced.

For each combination of source and detector there are tow physical measurements:

1. actual measurement

2. calibration measurement 
When a beam leaves the source in direction of detector, some part of beam which intersects the material or so called reconstruction region, traverse through a homogenous reference material such as air or water. At the end we have two sets of numbers for each of many source and detector position combinations and from these sets, CT numbers for the cross section of the object under investigation will be produced. When a material is placed between source and detector, depending on the energy of the photon and on the material, some of the photons will be absorbed or scattered i.e. they are removed from the beam.

The linear attenuation coefficient at certain energy depends on the probability of energy which is not absorbed or scattered and transmitted along the object. The relationship between attenuation coefficient and the probability is defined as follow:

$$
\mu_{\bar{e}}^{t}=-\ln \rho
$$

In which $\mu_{\bar{e}}^{t}$ is the linear attenuation coefficient of an object $t$ at energy $\bar{e}$, and $\rho$ is the probability that a photon of energy $\bar{e}$ which is not absorbed or scattered in the slab. The unit of linear attenuation coefficient is inverse length $\left[m^{-1}\right]$.

As it is mentioned before there are two sets of measurements for each combination of source and detector: calibration and actual measurement. Calibration measurements are taken from homogenous space around the object like water or air and actual measurements are taken from the object. It is assumed that the exterior of the reconstruction region is the same during the two sets of measurements.

In scanning a cross-sectional slice of an object, the slice, which has a thin thickness, is subdivided into small blocks with equal, square-shaped cross sections which are called volume elements or voxels. Roughly speaking, a $C T$ number is proportional to the average relative linear attenuation in a voxel. In the other word, according to the size of the object, there will be a $m \times n$ array of such voxels that exactly fills the reconstruction region and providing us with a $m \times n$ array of CT numbers. These numbers are used to display a $m \times \mathrm{n}$ array of small squares, with the uniform grayness in each one being proportional to the CT number of the voxel in the appropriate position. Note 
that relative linear attenuation coefficient is defined as $\mu_{\bar{e}}^{t}-\mu_{\bar{e}}^{a}$, where $t$ is the object occupying the point of space during the actual measurement and $a$ is the material occupying the during the calibration measurements.

As it is mentioned linear attenuation coefficient is the basic property which is measured in each voxel of CT image and this property is defined by Beers law. The assumption for Beers law is a narrow X-ray beam and monochromatic radiation whereas in practice CT spectrum is not monochromatic but polychromatic. The problem of polychromaticity is the introduction of imaging artefacts. For better understanding of the problem, first we should be aware of properties which attenuation coefficient is affected by. There are two basic issues which affect the attenuation at any points:

- Material at that point

- energy distribution of the beam (spectrum)

In CT the actual beam is polychromatic consisting of spectrum photons, i.e. spectrum is made up from many energy levels and it changes (hardens) when the beam passes through the object. This fact results in variation of the attenuation at a point with the direction of the beam passing through it whereas in case of monochromatic radiation in which the spectrum is made up from only one energy level, each point would have a uniquely assigned attenuation coefficient.

Figure B.1 illustrates attenuation and intensity relations in a monochromatic case:

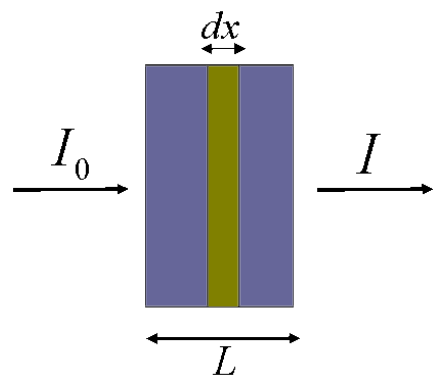

Figure B.1: The caption goes here

Energy transmitted along a particular ray path in a monochromatic case is driven as follows: 


$$
\begin{aligned}
& I(x+d x)-I(x)=-\mu(x) I(x) d x \\
& \Rightarrow \frac{I(x+d x)-I(x)}{d x}=-\mu(x) I(x) \\
& \Rightarrow \frac{d I}{d x}=-\mu I \Rightarrow \frac{1}{I} d I=-\mu d x \\
& \Rightarrow \ln I-\ln I_{0}=-\int_{0}^{x} \mu(x) d x \Rightarrow \ln \left(\frac{I}{I_{0}}\right)=-\int_{0}^{x} \mu(x) d x \\
& \Rightarrow I=I_{0} \exp \int_{0}^{x} \mu(x) d x
\end{aligned}
$$

\section{B.2 Line Integrals and Projection}

A cross section of an object is reconstructed from the projection data. Projections are a set of measurements of the integrated values of some parameters of the object. Fourier Slice Transform is the key to tomographic imaging and it relates the measured projection data to the two-dimensional Fourier transform of the object cross-section. Fourier-slice theorem is the basic relationship in analytic image reconstruction. This theorem states that that the Fourier transform of a one-dimensional projection is equivalent to a section, or profile, at the same angle through the center of the two-dimensional Fourier transform of the object.

As it is mentioned, projections are defined with line integrals which are the integral of some parameters of the object along a line. In this study we use $\mathrm{x}$ and $\mathrm{y}$ coordinates to represent the image and (theta,s) for the projection. Projection for a system showed above is expressed as follows:

$$
p(\theta, s)=\int_{0}^{L} f(x, y) d s=\ln \frac{I}{I_{0}}=\int_{s(x, y)} \mu(s(x, y)) d s
$$

where $s(x, y)$ are the coordinates of the attenuation coefficient in $x$ and $y$ dimensions, $L$ is the path length from source to detector and $d s$ is a distance along this path length.

All these equations express the relationships between intensity and attenuation coefficient for monochromatic radiation whereas the CT scanner uses polychromatic radiation so the Beers law should be corrected for polychromatic radiation to avoid imaging artefacts. 
The true situation can be expressed by following equation, which represents attenuation coefficient as a function of energy and position.

$$
I=\int_{e_{l}}^{e_{h}} \frac{d I_{0}}{d E} \varepsilon(E) \exp \left(-\int_{0}^{h} \mu(x, y, E) d L\right) d E
$$

where the variables are defined as follows:

$\frac{d I_{0}}{d E}$ : spectral distribution of the incident radiation

$\varepsilon(E)$ : efficiency of the detector at a particular energy $\mathrm{E}$

$e_{l}$ : relevant spectrum of low energy

$e_{h}$ : relevant spectrum of high energy

\section{B.3 Image reconstruction}

As it is mentioned in previous section, the image of an object is reconstructed from line integral of a property of the image, known as projection. Image reconstruction in $\mathrm{CT}$ is a mathematical process that generates images from X-ray projection data acquired at many different angles around the patient. Image reconstruction has a fundamental impact on image quality and therefore on radiation dose. For a given radiation dose it is desirable to reconstruct images with the lowest possible noise without sacrificing image accuracy and spatial resolution.

There are different methods for recovering the image of the cross section of an object from the projection data. Two major categories of methods exist, analytical reconstruction and iterative reconstruction. Two methods for image reconstruction are explained in this study:

1. Algebraic methods

2. 2D Radon transforms

Algebraic method is an iterative reconstruction and 2D radon transform is a method of analytical reconstruction.

\section{B.3.1 Algebraic method}

In this approach for tomographic imaging, it is assumed that the crosssection consists of an array of unknowns, and algebraic equations should be 
setting up for the unknowns in terms of the measured projection data. To be taken into consideration that although this method has a simple and straight procedure but is not appropriate for medical CT due to low accuracy and low speed of implementation.

The algebraic methods are often called iterative methods because they compute reconstructed images by using iterative methods. This method is used to reconstruct $2 \mathrm{D}$ and $3 \mathrm{D}$ image from the projection and it was the one first used by Hounsfield.

There are a large variety of algorithms, but all of them solved with the methods that successively improve and iterate an estimate of the unknowns, i.e. each of the algorithm follows a procedure as follows:

- starts with an assumed image

- computes projections from the image

- Compares the original projection data

- Update the image based upon the difference between calculated and the actual projections

In Algebraic method, with respect to the number of pixels, number of linear equations should be solved and iterates till an appropriate image is reconstructed. Figure B.2 is a schematic illustration of an object subdevided to four pixeles.

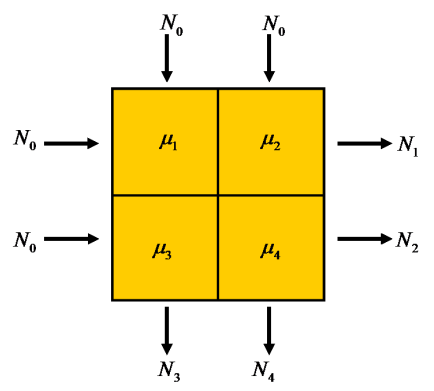

Figure B.2: Simplified system for image reconstruction with algebraic method 
For such a system shown above, the following system of equations should be solved by iterative reconstruction in order to determine the attenuation coefficient for each pixel.

$$
\left\{\begin{array}{l}
N_{1}=N_{0} e^{-\left(\mu_{1}+\mu_{2}\right) x} \\
N_{1}=N_{0} e^{-\left(\mu_{1}+\mu_{2}\right) x} \\
N_{1}=N_{0} e^{-\left(\mu_{1}+\mu_{2}\right) x} \\
N_{1}=N_{0} e^{-\left(\mu_{1}+\mu_{2}\right) x}
\end{array}\right.
$$

\section{B.3.2 Radon transform}

The Radon transform is an integral transform whose inverse is used to reconstruct images from medical CT scans.

A Radon transform or a sinogram of a distribution $f(x, y)$ is given by

$$
p(\theta, s)=\iint f(x, y) \delta(x \cos \theta+y \sin \theta-s) d x d y
$$

Coordinates $x, y, s$ and $\theta$ are defined in figure B.3.

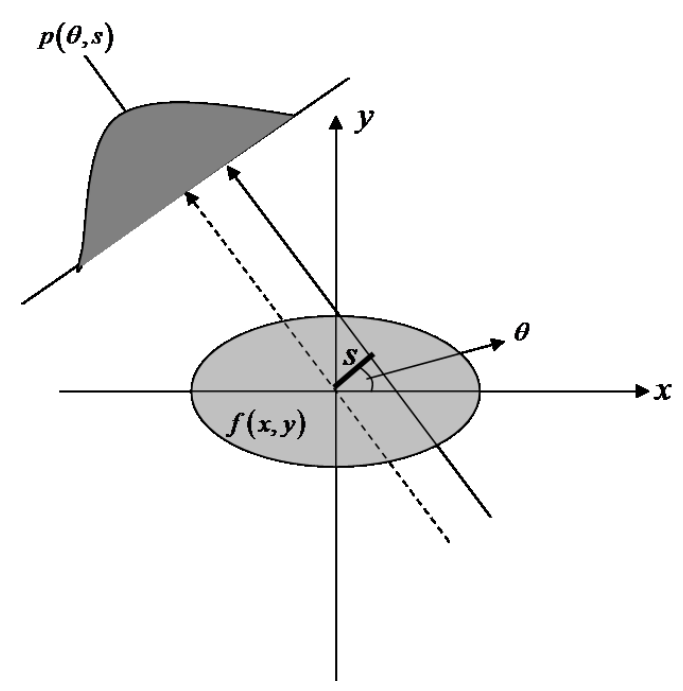

Figure B.3: Illustration of coordinates for an object and its projection

A projection $p(\theta, s)$ is formed from integration along all parallel lines at an angle $\theta \cdot p(\theta, s)$ is defined as a 1-D projection at an angle $\theta$ and it is the 
line integral of the image intensity along a line $l$. The projections are organized into a sinogram such that each complete projection fills a single row of $\theta$ in the sinogram. In this format, a single point in $f(x, y)$ traces a sinusoid in the sinogram. The function $p(\theta, s)$ is often referred to as a sinogram because the Randon transform of an off-center point source is a sinusoid.

Sinogram is a two dimensional function of $s$ and $\theta$, which is formed by the collection of all projections for $0<\theta<2 \pi$. A fixed point in the object traces a sinusoidal path in the projection.

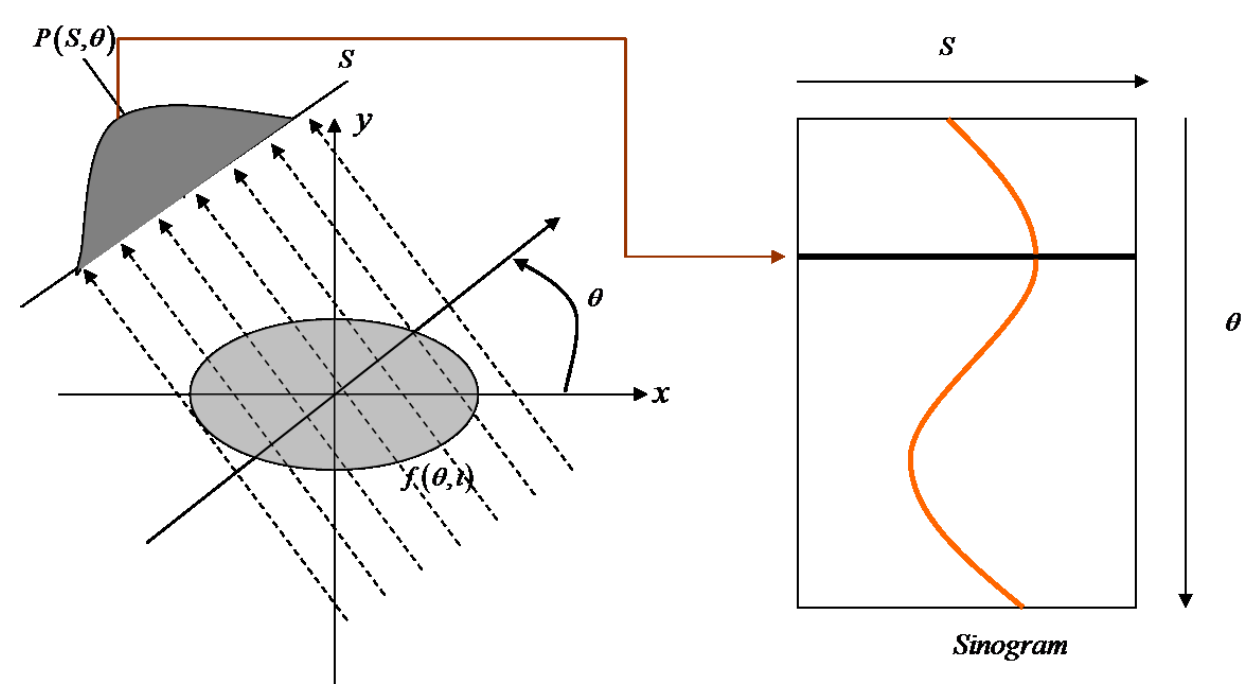

Figure B.4: The Image and its projection at specified angle and the related sinogram 
The object $f(x, y)$ is converted to the projection $p(\theta, s)$ by a line-integral transformed which is called the X-ray transform, the detailed equations for this transform are provided in the previous chapter. This transform in $2 \mathrm{D}$ is same as Radon transform.

As it is mentioned, in order to reconstruct the images, the inversion of the Radon transform should be determined. The solution of the inverse Radon transform is based on the Fourier Transform Theorem, also known as Central Slice Theorem. The Fourier Slice Theorem states that the Fourier Transform of a one-dimensional projection $p(\theta, s)$ is equal to the 2D Fourier Transform of the image $f(x, y)$ evaluated at the same angle as the projection was taken. In the other word, the Fourier Slice Theorem relates the two-dimensional Fourier transform of image, $F_{2}\{f(x, y)\}$, and the one-dimensional Fourier transform of projections, $F_{1}\{p(\theta, s)\}$.

The image, $f(x, y)$, is transformed to $F\left(v_{x}, v_{y}\right)$ using two-dimensional Fourier transform, and projection, $p(\theta, s)$, is transformed to $P\left(\theta, v_{s}\right)$ by onedimensional Fourier transform, where $v_{s}$ is the Fourier space conjugate of $x$. The central-section theorem indicates that if we know the projections, sinograms, at all angles $0<\theta<2 \pi$, then it is possible to find the values for $F_{1}\{p(s, \theta)\}$. The inverse two-dimensional Fourier transform of $F_{1}\{p(\theta, s)\}$ will result in $f(x, y)$.

All these explanations for Fourier-slice theorem and image reconstruction are schematically illustrated in figure B.5,

Fourier Slice Theorem can be shown mathematically in the following way, but before that line integrals and projections should be determined. Line integrals, as its name implies, represents the integral of some parameter of the object along a line, this parameter could be the attenuation of x-rays as they propagate through the object. The line integral represents the total attenuation suffered by a beam of x-rayss as it travels in a straight line through the object.

In following calculation the object is represents by a two-dimensional function $f(x, y)$ and each line integral by $(\theta, s)$ parameters. Note that all points on this line satisfy the equation:

$$
s=x \cos \theta+y \sin \theta
$$




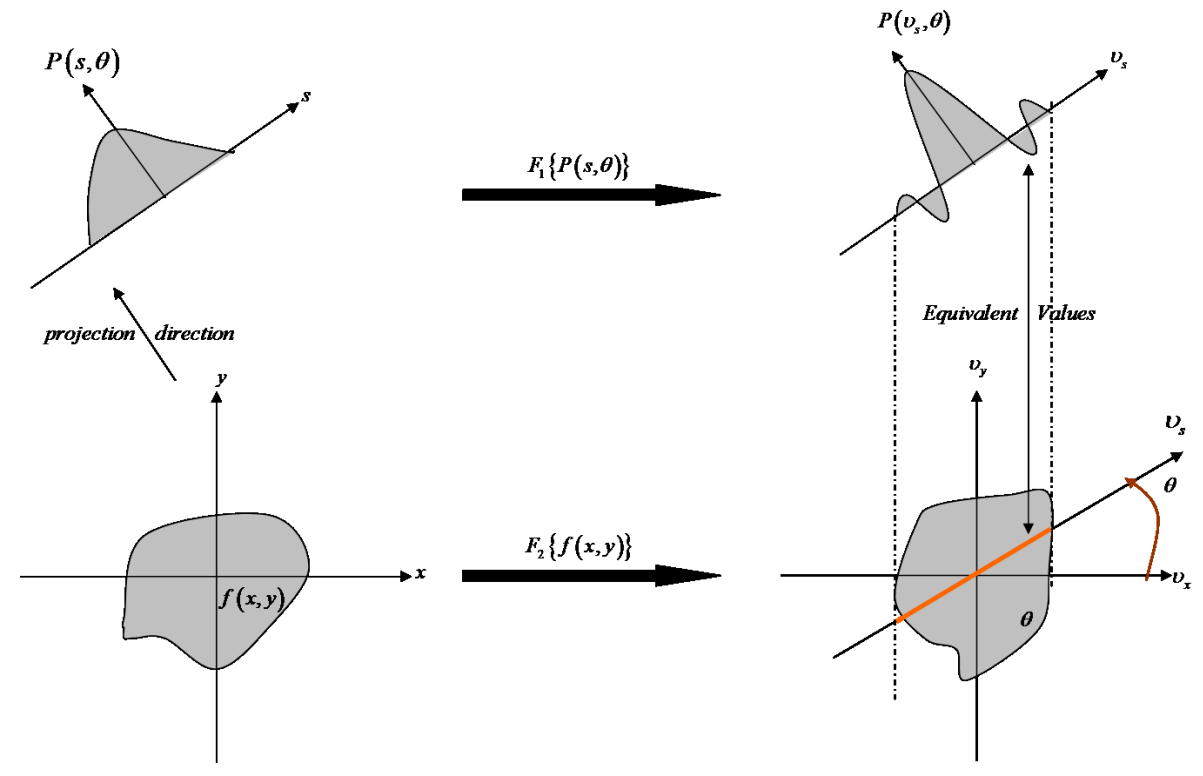

Figure B.5: Schematic illustration of Fourier Slice Theorem and Radon Transform

Projection of the image can be expressed as

$$
p(\theta, s)=\int_{(\theta, s) l i n e} f(x, y) d l
$$

Using a delta function, this can be rewritten as

$$
p(\theta, s)=\iint f(x, y) \delta(x \cos \theta+y \sin \theta-s) d x d y
$$

This function is known as the Radon transform of the function $f(x, y)$.

As it is mentioned, a projection consists of a combination of a set of line integrals. In this study we show the simplest projection which is a collection of parallel ray integrals and it is denoted by $p(\theta, s)$ for a constant $\theta$. It could be measured by moving an x-ray source along parallel lines on opposite sides of an object.

After defining the line integrals and projection, we can drive the Fourier Slice Theorem. 
One-dimensional Fourier transform of aprojection, $\mathrm{p}($ teta,s) at an angle is defined as

$$
P\left(\theta, v_{s}\right)=\int e^{-j 2 \pi v_{s}} p(\theta, s) d s
$$

Substitution of definition of $p(\theta, s)$ in the equation of the one-dimensional Fourier transform of $p(\theta, s)$ results in

$$
P\left(\theta, v_{s}\right)=\iiint f(x, y) e^{-j 2 \pi v_{s} s} \delta(x \cos \theta+y \sin \theta-s) d x d y
$$

Using Dirac delta function simplify this equation to

$$
P\left(\theta, v_{s}\right)=\iint f(x, y) e^{-j 2 \pi v_{s}(x \cos \theta+y \sin \theta-s)} d x d y
$$

Now, if we recall the definition of the two-dimensional Fourier transform of image $f(x, y)$, we would have

$$
F\left(v_{x}, v_{y}\right)=\iint f(x, y) e^{-j 2 \pi\left(v_{x} x+v_{y} y\right)} d x d y
$$

We can see that equation $a$ is equal to $F\left(v_{x}, v_{y}\right)$ evaluated at $v_{x}=v_{s} \sin (\theta)$ and $v_{y}=-v_{s} \operatorname{cps}(\theta)$, which is the line that the projection $p(\theta, s)$ was taken on, or in the other word

$$
P\left(\theta, v_{s}\right)=F\left(v_{s}, \theta\right)=F\left(v_{s} \cos \theta, v_{s} \sin \theta\right)
$$

This equation is the essence of straight ray tomography and proves the Fourier Slice theorem.

Up to here the Fourier Slice Theorem is driven by taking the one-dimensional Fourier transform of a parallel projection and noting that it is equal to a slice of the two-dimensional Fourier transform of the original object. Now it is possible to reconstruct the image by simply performing a two-dimensional Fourier transform of the object. Inverse of the two-dimensional Fourier transform of $f(x, y)$ is given by following equation

$$
f(x, y)=\frac{1}{4 \pi^{2}} \iint F\left(v_{x}, v_{y}\right) e^{j\left(v_{x} x+v_{y} y\right)} d x d y
$$

Changing the variables from rectangular to polar coordinates and replacing $F\left(v_{s}, \theta\right)$ with $P\left(v_{s}, \theta\right)$ results in

$$
f(x, y)=\frac{1}{4 \pi^{2}} \iint P\left(v_{s}, \theta\right) e^{j v_{s}(x \cos \theta+y \sin \theta)}\left|v_{s}\right| d v_{s} d \theta
$$


where $\left|v_{s}\right|$ is the determinant of the Jacobian of the change of the variable from rectangular to polar coordinates. We now have a relationship between the projection function and the image and it we are able to reconstruct the image.

\section{B.4 CT numbers}

After image reconstruction, relative values of attenuation coefficient are known for each pixel. The output of CT scanner is matrices of attenuation coefficient represents in Hounsfield unit. CT numbers are values which is produced by conversion attenuation doefficients into corresponding numerical values by normalizing with linear attenuation coefficient of water, $\mu_{w}$. The relationship between attenuation coefficient and Hounsfield is expressed as follows.

$$
C T=1000 \frac{\left(\mu-\mu_{w}\right)}{\mu_{w}}
$$

The unit of CT numbers is Hounsfield unit HU. $\mu_{w}$ is attenuation coefficient of water $\left[\mathrm{m}^{-1}\right], \mu$ is local attenuation coefficient averaged over a voxel in $\left[m^{-1}\right]$. Each Hounsfield unit represents $0.1 \%$ change in density with respect to calibration density scale, water in this case. CT number increases as density of the object increase, because the linear attenuation coefficient is a function of density.

It is possible to determine the saturation of a liquid in a core from CT data using following equation:

$$
S_{\text {liquid }}=\frac{C T_{\text {exp }}-C T_{d r y}}{C T_{\text {wet }}-C T d r y}
$$

where $S_{\text {liquid }}$ is liquid saturation in the core, $C T_{\exp }$ is the measured CT number during the experiment, $C T_{d r y}$ is the $\mathrm{CT}$ number for dry core and $C T_{\text {wet }}$ is the CT number for fully saturated core. To be taken into consideration, using subtraction algorithm eliminated the effect of rock on the total measured attenuation. 


\section{Appendix $\mathrm{C}$}

\section{Saturation Procedure}

The saturation procedure is as follows,

1. Core is heated up to remove all the containing water,

2. Core is evacuated,

3. To remove air, core is filled with $\mathrm{CO}_{2}$ up to a certain pressure and the core is then evacuated. This procedure is repeated several times,

4. About 5 pore volumes of oil are pumped through the core. $\mathrm{CO}_{2}$ dissolves in the oil and removed from the core.

For saturating the core with eicosane, which is a solid material at room temperature, all the equipments are placed inside a thermostatic bath to provide an appropriate temperature for melting the eicosane. 


\section{Acknowledgements}

First and foremost I would like to offer my sincerest gratitude to my supervisor Professor Hans Bruning who has earnestly supported me throughout my thesis with his exemplary patience and vast knowledge, whilst giving me the opportunity to work in my own way. I also would like to thank Dr. Rouhi Farajzadeh who taught me so many things not only about the science but also about the life. I attribute the level of my Masters degree to his encouragement and effort, without whom this thesis would not have been completed or written. One simply could not wish for a better environment or friendlier supervisors.

I am also deeply indebted to Dr. Susanne Rudolph for her gracious manners and enlightening advices. Her heartwarming good will infused me with self-conffidence and determination. I would like also to thank Mohammad Simjoo for the brilliant discussions and comments and also for helping me to use the CT-scanner.

The technicians of Dietz lab: Karel Heller and Ellen Meijvogel-de Koning have to be solemnly acknowledged. I could not even conduct a single experiment without their assistance.

I also appreciate Amin Amerighasrodashti who provided useful arguments about my thesis and helped me to initiate my work.

My special thanks to the people in Shell: Dr. Diedrik Boersma who gave me the opportunity to work in the Shell's office; Dr. Sander Suicmez for his invaluable and generous supports also his precious comments and guidance during my working period in Shell; Stefan Berg for helping me in CT data analysis. 
I shall express my deepest gratitude to my parents without whose unconditional, selfless and perpetual supports I would not be even close to where I am now.

Last but not least, all my benevolent friends and companions, Armin, Pejman, Farid, Farzad, Roozbeh, Rahul, Efe, Aydin, Siamak and Nami, whose help sustained me with hope and passion during my studies in the Netherlands have to be particularly thanked. 